\title{
On turbulent dynamics and related theoretical topics associated with diffusive incompressible fluid models
}

\author{
Zachary Bradshaw \\ Charlottesville, Virginia
}

B.S. Mathematics, Virginia Commonwealth University, 2009

B.S. Computer Science, Virginia Commonwealth University, 2009

A Dissertation presented to the Graduate Faculty of the University of Virginia in Candidacy for the Degree of Doctor of Philosophy

Department of Mathematics

University of Virginia

May, 2014 


\begin{abstract}
This dissertation aims to establish rigorous results concerning the behavior of diffusive or fractionally diffusive fluid regimes and identify connections to and implications for theoretical topics relating to the possible irregularity of the corresponding mathematical models. The particular models of interest are the three dimensional Navier-Stokes equations, the magnetohydrodynamical equations, and the fractionally diffusive surface quasi-geostrophic equations.

Two issues concerning turbulent transport are examined. The first relates to the characterization and existence of turbulent cascades across inertial ranges in viscous (or fractionally diffusive) fluid systems and amounts to providing rigorous support for physically and numerically motivated descriptions of turbulent media. The second aims to establish sharp lower bounds for the dissipative length scales as these play an important role in turbulent transport dynamics and have applications to conditional regularity criteria involving anisotropic diffusion of the transverse length scales associated with coherent vortex structures.

The regularity problems associated with these models are challenging and generally remain open. An important issue in this area relates to the fact that the considered models are supercritical. Frequently, to obtain regularity criteria in such a setting, conditions must be included to render this supercritical behavior critical or, better yet, subcritical. Several results along these lines are included which are physically motivated by the anitropic filamentary description of turbulent fluids.
\end{abstract}




\section{Acknowledgements}

I am deeply grateful too...

Zoran Grujić, who has been a source of inspiration throughout my graduate career. I have benefited tremendously from his breadth of vision, intellectual generosity, and willingness to provide thoughtful and meaningful guidance.

My mother, Ann, for instilling in me curiosity and determination.

My sister, Amy, for supporting me when my determination was challenged.

Professor Irena Lasiecka, who has been an encouraging teacher.

Professor Roberto Triggiani, for his ubiquitous honesty.

My friends and colleagues.

My dog, Pox, although she cannot read this.

Z.B. was partially supported by the Virginia Space Grant Consortium via a Graduate Research Fellowship for the 2012-2013 and 2013-2014 academic years. Z.B. also received support from the National Science Foundation grant NSF DMS 1212023.

Dedicated to Matt King. 


\section{Contents}

1 Introduction $\quad 1$

1.1 Fluid models ................... 3

1.2 Turbulence in viscous, incompressible fluids . . . . . . . 6

1.3 Anisotropic diffusion and regularity . . . . . . . . . . . 12

2 Cascades and transport processes in astronomical and geophysical turbulence $\quad 15$

2.1 Introduction . . . . . . . . . . . . . . . . . . . 15

2.2 Energy cascades and related processes in astronomical plasmas . . . . . . . . . . . . . . . . . . . . . 18

2.2.1 $\left(K_{1}, K_{2}\right)$-covers and ensemble averages . . . . 20

2.2.2 3D incompressible MHD equations . . . . . . . 25

2.2.3 Total energy cascade . . . . . . . . . . . . . 27

2.2.4 Cascade-like dynamics of the total fluid energy . . 34

2.2.5 Other cascade-like dynamics . . . . . . . . . . . 42

2.2.6 Locality of the energy flux . . . . . . . . . . . . 48

2.2.7 A scenario exhibiting predominant $u$-to- $b$ energy transfer ................ . 49

2.3 Enstrophy transport and concentration in astronomical plasmas .......................... 51

2.3.1 Enstrophy concentration . . . . . . . . . 55

2.3.2 Estimates . . . . . . . . . . . . . . 63

2.3.3 Proof of Theorem 2.3.1 . . . . . . . . . . . . 74

2.3.4 Locality of the enstrophy flux . . . . . . . . . 77

2.4 On the temperature variance cascade in SQG turbulence 78 2.4.1 Methodology . . . . . . . . . . . . . 81

2.4.2 The localized temperature variance flux . . . . . . 84

2.4.3 The temperature variance cascade . . . . . . . 86 
3 Uniform analyticity radii and anisotropic diffusion $\quad 91$

3.1 Introduction . . . . . . . . . . . . . . . . . . . 91

3.2 Sharp lower bounds on the analyticity radii of solutions to 3D MHD with $L^{\infty}$ initial data . . . . . . . . . . . 93

3.3 A geometric measure-type regularity criteria for 3D MHD 107 3.3.1 Requisite material . . . . . . . . . . . . . . . . . 109

3.3 .2 Regularity criteria . . . . . . . . . . . . . . . 110

3.4 Local estimates for the analyticity radius of solutions of the Navier-Stokes equations with locally analytic forcing 118 3.4.1 Estimates in $L^{s}$ for $P_{\alpha}^{(n)}(t)$ and $\Pi_{\alpha}^{(n)}(t) \ldots 125$ 3.4.2 Inductive estimates in $L^{q}$ for $U_{\alpha}^{(n)}$ and $V_{\alpha}^{(n)} \ldots 128$

3.4.3 Proof of Theorem 3.4.1 . . . . . . . . . . . 138

4 Logarithmically subcritical scenarios for solutions to the 3D Navier-Stokes equations 139

4.1 Introduction . . . . . . . . . . . . . . . . . . . 139

4.2 Preliminaries from harmonic analysis . . . . . . . . . 143

4.3 Blow-up scenarios exhibiting logarithmically subcritical anisotropic diffusion . . . . . . . . . . . . . . . . . 148 4.3.1 Amenable blow-up rates . . . . . . . . . . . . . 148 4.3 .2 Main result . . . . . . . . . . . . . . . . 155

4.4 A geometric scenario exhibiting logarithmically subcritical anisotropic diffusion . . . . . . . . . . . . 166 


\section{Chapter 1}

\section{Introduction}

Turbulence is simultaneously a remarkably common and profoundly complex phenomenon. It is an ubiquitous feature of fluids occupying the world around us and beyond; indeed, the vast majority of the universe's material mass takes the form of highly turbulent astronomical plasmas [8]. These physical systems are thought to obey various systems of partial differential equations such as the Navier-Stokes equations (NSE) which describe viscous, incompressible flow, the magnetohydrodynamic equations (MHD) which describes a coupled system consisting of a magnetic field and an interacting charged fluid, and, in constrained geophysical contexts, the surface quasi-geostrophic equations (SQG). A concrete mathematical understanding of these models is important as they provide indispensable insight for engineers and scientists across a wide range of disciplines and applications such as nautical and aerospace engineering, meteorology and oceanography, climate science, magnetic confinement of plasmas in nuclear reactors, solar wind turbulence and plasma dynamos, etc.

These models are also interesting from a mathematical standpoint. An important open question is that of the global-in-time existence and smoothness of weak solutions to 3D NSE (or, alternatively, the breakdown of such solutions) which constitutes a Millennium Problem posed by the Clay Mathematics Institute. The global-in-time well-posedness of other fluid models such as MHD or supercritical SQG also remain unsolved. Considering the difficulty of these problems it is natural to seek conditional regularity results based on physically reasonable criteria e.g. observational and numerical studies of turbulence - to illuminate the nature of the models.

There are a variety of ways in which mathematical analysis can 
improve our understanding of these fluid models. Two such themes are apparent in this dissertation.

The first of these lies in the use of mathematical analysis to characterize and confirm observationally and numerically established features of turbulent fluids as intrinsic properties of the governing systems. Effectively, this approach looks to mathematics to better understand physically based descriptions of fluid mechanics. This approach is apparent in Chapter 2 as well as Chapter 3 (see Sections 3.2 and 3.4) where physically significant properties of turbulent fluids are investigated from a rigorous perspective.

The second applies the physically based knowledge of fluid dynamics to theoretical issues, e.g. developing physically motivated conditional regularity criteria. Work in this spirit is contained in Chapter 3 (see in particular Section 3.3) and Chapter 4.

In the remainder of this Chapter we first introduce the fluid models which will be of primary interest to us and proceed to give some impression of the topics relating to them that this dissertation considers.

\section{$1.1 \quad$ Fluid models}

The Navier-Stokes equations describe the motion of a viscous, incompressible fluid and is important for its role in practical applications as well as its mathematical significance. Working in $\mathbb{R}^{3}$ with initial data $u_{0}$ taken in an appropriate function space - typically this is the divergence free subspace $H$ of the energy space $L^{2}\left(\mathbb{R}^{3}\right)$ but can vary depending on context - the velocity field $u$ of a viscous, incompressible fluid and an associated scalar pressure $p$ satisfy the system,

$$
\left\{\begin{array}{l}
\partial_{t} u+(u \cdot \nabla) u=-\nabla p+\nu \Delta u+f \\
\nabla \cdot u=0 ; \quad u(x, 0)=u_{0}
\end{array}\right.
$$

where $\nu$ is the kinematic viscosity and $f$ is a divergence free forcing term. The divergence free condition $\nabla \cdot u=0$ characterizes the fluid as incompressible while $\nu>0$ indicates the fluid is viscous. A consequence of incompressibility can be seen by taking the divergence of the velocity equation to obtain,

$$
-\Delta p=\partial_{i} \partial_{j}\left(u_{i} u_{j}\right)
$$


Thus, the pressure is determined kinematically (modulo a harmonic function) as the solution of an elliptic system and in typical circumstances can be recovered from the velocity via singular integral operators.

Alternatively, one can focus on the evolution of the vorticity $\omega=$ $\nabla \times u$ which is obtained from the above system by applying the curl operator throughout and takes the form,

$$
\left\{\begin{array}{l}
\partial_{t} \omega+(u \cdot \nabla) \omega=(\omega \cdot \nabla) u+\nu \Delta \omega, \\
\nabla \cdot \omega=0 ; \quad \omega_{0}=\nabla \times u_{0} .
\end{array}\right.
$$

Again, incompressibility leads us to an elliptic relationship,

$$
-\Delta u=\nabla \times \omega,
$$

and, under minimal assumptions on the flow (e.g. those given in [32]), we can express the velocity field in terms of singular integral operators applied to the vorticity.

A rich mathematical literature exists regarding NSE (cf. [35, 81, 104] for foundational expositions). We forgo a general survey of the historical development of the field as it is beyond the scope of this document but, when describing our results, we will comment further on the most relevant existing research.

The Navier-Stokes equations can be refined in certain contexts to yield other fluid models. An example of this is the coupling of 3D NSE and Maxwell's equations through the Lorentz force which leads to an evolutionary system for plasma flows - i.e. fluid media comprised of charged particles - and an interacting magnetic field. The resulting partial differential equations are known as the magnetohydrodynamic system and are important for the study of astronomical plasmas such as stellar winds, the interstellar medium, and accretion disks. More precisely, MHD describes the evolution of the plasma's velocity field $u$ and an interacting magnetic field $b$ via the equations,

$$
\left\{\begin{array}{l}
u_{t}-\nu \Delta u+(u \cdot \nabla) u-(b \cdot \nabla) b+\nabla\left(p+|b|^{2} / 2\right)=0, \\
b_{t}-\eta \Delta b+(u \cdot \nabla) b-(b \cdot \nabla) u=0, \\
\nabla \cdot u=\nabla \cdot b=0, \\
u(x, 0)=u_{0}(x) ; \quad b(x, 0)=b_{0}(x),
\end{array}\right.
$$

where $\eta$ and $\nu$ are the magnetic resistivity and kinematic viscosity respectively and $p$ is the fluid pressure. In comparison to NSE, $u$ and 
$b$ are both divergence free and essentially non-linear perturbations of heat equations, and, consequently, similar connections can be drawn between these fields, the pressure, and the vorticity and current fields $\omega=\nabla \times u$ and $j=\nabla \times b$. The degree to which these formal similarities extend to the dynamical behavior of real world phenomena is, however, more subtle, a fact reflecting the more complicated transport terms.

Another refined model is the surface quasi-geostrophic system (SQG) which was introduced in the context of the quasi-geostrophic equations (QG), a geophysical 3D model for the displacement of a rotating, stratified fluid from a solid body revolution, to address the fact that the QG description of atmospheric and oceanic processes breaks down in regions near the boundaries of certain geophysical fluids like the troposphere of the atmosphere and the ocean surface. To capture the dynamical characteristics of these regimes, QG is augmented with an assumption that the vorticity potential is uniform, a modification which leads to SQG. Technically speaking, the SQG equations govern the evolution of fluid's surface temperature $\theta$ which is subjected to inertial effects and dissipative forces. The fluid velocity $u$ and the surface temperature are coupled through a streamfunction $\Psi$ which is assumed to be independent of the vertical coordinate. Over a flat surface, taken to be $\mathbb{R}^{2}$, the system has the form,

$$
\begin{cases}\left(\partial_{t}+u \cdot \nabla+\kappa \Lambda^{\alpha}\right) \theta=0, & u=\left(-\partial_{2} \Psi, \partial_{1} \Psi\right) \\ \theta(\cdot, 0)=\theta_{0}, & \Lambda \Psi=-\theta\end{cases}
$$

where $0<\alpha \leq 2, \kappa \geq 0$, and $\theta_{0}$ is a scalar valued initial datum taken in an appropriate function space. The dissipative operator $\Lambda^{\alpha}$ is a Fourier multiplier determined by,

$$
\left(\Lambda^{\alpha} f\right)^{\wedge}(\xi)=|\xi|^{\alpha} f^{\wedge}(\xi)
$$

If $\kappa>0$ then values of $\alpha$ where $0<\alpha<1, \alpha=1$, or $1<\alpha \leq 2$ correspond to formulations of SQG which behave distinctly and are respectively referred to as supercritical, critical, and subcritical. These three regimes are connected to the well-posedness of the corresponding system and can be seen as a struggle between the diffusive smoothing effects of the fractional Laplacian versus the nonlinear effect of the transport term. 


\subsection{Turbulence in viscous, incompressible fluids}

Turbulence theories have traditionally focused on providing a precise characterization of the dynamics of turbulent flows. Despite a chaotic appearance, observational and numerical studies indicate that certain regular processes transpire involving the creation, evolution, and destruction of coherent structures whether they be vortexes in 3D hydrodynamics, current sheets in 3D magnetohydrodyanamics, or temperature variance assemblages in surface quasi-geostrophic dynamics. For mathematicians, challenges lie in rigorously affirming the ubiquity of certain observationally apparent dynamics as well as providing more precise estimates for physically significant quantities working directly from the governing equations.

The dynamical processes of chief interest to us are the turbulent cascades, i.e. the net, inertially driven transport of an ideally conserved quantity from larger to smaller scale structures (cf. [28, 55, 8]). In the three dimensional NSE energy cascade, for example, energy is injected by a source at a "macro scale" and initially concentrates on vortex filaments of approximately this scale. As turbulence evolves, energy is transported by inertial effects from larger to smaller structures, that is, it cascades. This transport process ceases at a dissipative scale where inertial effects are outweighed by viscous forces and frictional diffusion interferes with inertial transport dynamics. The dissipative scale can be realized mathematically as the uniform analyticity radius of the flow [55]. The range of scales between the macro and dissipative scales is called the inertial range as, across it, inertial forces dominate. Our work in this area focuses on two topics and we presently remark on these.

(1) The first class of results is contained in Chapter 2 where we examine the existence of certain cascades and related transport phenomena in MHD and SQG turbulence.

In 3D MHD turbulence, the total energy - i.e. the sum of magnetic, kinetic, and potential energies - exhibits a direct cascade, a fact broadly supported by numerical and observational evidence (for several modern, numerically motivated spectral phenomenologies see $[9,12,90,93])$. In [15], Z.B. and Z. Grujić established conditions under

which, in a statistically significant sense, the energy flux is directed 
from larger to smaller scales across an inertial range and, furthermore, is nearly constant. These results are carefully presented in Chapter 2.2. Our conclusions follow directly from the 3D MHD equations under assumptions which are physically reasonable for many turbulent plasmas and, in particular, astronomical plasmas such as the solar wind and the interstellar medium. In contrast to the current phenomenological theories, the results do not appeal to the existence of a strong magnetic guide field which indicates that the energy cascade is an intrinsic property of the 3D MHD system and not solely an artifact of the plasma's environment.

A related dynamical process in 3D MHD turbulence reflects a picture of intermittently distributed regions of high spatial complexity, i.e. current sheets, which become increasingly thin as turbulence evolves $[8,19,59,60,94]$. One view is that this process is initiated and driven by inertial effects associated with the cascades of ideally conserved quantities (see [48, 82, 97, 110]; for an alternative view, see [13, 19]). This is visible in the progressive concentration of enstrophy, i.e. the sum of the squares of vorticity and current, on small-scale current sheets. Understanding this concentrative process is important as it is connected to the development of current-driven instabilities, e.g. the tearing instability (cf. [8]), which drives magnetic reconnection (the relationship between magnetic reconnection and current structures has been studied in $[48,94])$. In [16] and Chapter 2.3, Z.B. and Z. Grujić identify conditions under which the picture of concentrative inertial transport of enstrophy is affirmed. In the case that non-inertial effects are negligible at large scales, this indicates a detectable large-to-small scale transport of enstrophy which is cascade-like. In this scenario, then, inertial forces have a morphological effect on current sheets and, therefore, the advection of these structures is likely not entirely passive.

A spectral theory of SQG turbulence was initially considered by Blumen in [11] where he derives Kolmogorov-Kraichnan type scaling laws and associated inertial ranges (see also [67]). Here, there are two ideally conserved quantities, the surface temperature variance and the depthintegrated total energy, and their respective densities are $|\theta|^{2}$ and $|u|^{2}$. In a submitted work which is also described in Chapter 2.4, Z.B. and Z. Grujić provide a rigorous affirmation of the existence of a forward temperature variance cascade across an inertial range under conditions which are consistent with qualitative properties of turbulence. The con- 
dition triggering the cascade is interesting as it must accommodate the inherent non-locality introduced by fractional diffusion. To overcome this, a non-local extension operator $L$ (which has been studied extensively in [21]) is incorporated through which the fractional Laplacian of the temperature variance on $\mathbb{R}^{2}$ can be recovered as the trace of the normal derivative of $L \theta$. Using this, we provide a formula for the local temperature variance flux which is akin to local inequalities developed in [22] and [39].

(2) The second topic of interest to us which relates to turbulent cascades involves determining sharp lower bounds for the uniform analyticity radius - recall that this length is connected to the scale at which diffusive effects outweigh the inertial mechanisms which drive the cascade - associated with certain fluids and is contained in Chapter 3. Such bounds for solutions to NSE and related models have been developed in a variety of settings using several theoretical devices. For viscous fluids, these results frequently depend on Fourier methods and are restricted to the whole space or the torus. A pioneering work along these lines is that of Foias and Temam (cf. [54]) where it is shown that certain solutions become Gevrey-regular at times $t>0$ and, moreover, the uniform analyticity radius increases with time. Another approach was developed by Grujić and Kukavica and is carried out in physical space using $L^{p}\left(\mathbb{R}^{n}\right)$ norms, cf. [62, 76], and, in the limit of $L^{\infty}$ initial data (cf. [64]), has implications for geometric-measure based regularity criteria as highlighted in [61].

Chapter 3 contains an extension of the $L^{\infty}\left(\mathbb{R}^{3}\right)$ approach to $3 \mathrm{D}$ MHD with a conditional regularity criteria in mind (these results are also contained in [14]). The regularity criteria considers the superlevel sets of the magnitudes of the velocity and magnetic fields and is essentially a requirement that these sets be sparse in a suitable, lower dimensional sense. Several formulations of the criteria are included and are consistent with the observation that the velocity field plays a more important role as far as regularity is concerned than the magnetic field (this reflects the the formal structure of the induction equation which is effectively linear in $b$ ).

Interestingly, the $L^{p}$ approach developed by Grujić and Kukavica in [62] can be extended to accommodate local forcing. A proof of concept for this idea is illustrated in [63] where they study the problem for a nonlinear heat equation on a bounded domain with Dirichlet boundary 
conditions. In Chapter 3.4 we use a structurally similar approach to [63] to locally estimate the analyticity radius of solutions to NSE on $\mathbb{R}^{3}$ with locally real analytic forcing - these results reflect an ongoing collaboration between Z.B., Z. Grujić, and I. Kukavica. The work in [63] is comparably more direct than the considered problem because, there, the nonlinear contribution to the heat equation is local and involves no derivatives. This contrasts the Navier-Stokes equations where the pressure constitutes a non-local effect on the evolution of the velocity and the transport term involves a differential component. Despite these complications sharp lower bounds can still be established. Our work differs further from that of [63] which is built around energy functional estimates as our technique is based on an iterative approximation argument going back to Kato's semigroup approach to strong solutions (cf. [72]). For our purposes, though, we must consider an approximation scheme which involves recursively solving second order linear heat equations with non-incompressible forcing and our techniques are considerably more involved than the estimates of [72] or [62] (the latter reference also extends the approach of [72] but does so in a global, and thus less messy, context).

\subsection{Anisotropic diffusion and regularity}

The second theme mentioned at the outset reflects the aim of developing physically motivated conditional regularity criteria and this characterizes the aims of Chapter 4. To highlight our efforts there, note that a "scaling-gap" exists between known a priori finite quantities, take, for instance, $\sup _{0<t<T}\|u\|_{2}^{2}$ or $\sup _{0<t<T} \mid\|\nabla u\|_{1}$, and quantities with respect to which regularity can be conditioned, e.g. $\sup _{0<t<T}\|u\|_{3}^{3}$ or $\sup _{0<t<T}|| \nabla u \|_{2}^{2}$. Under the natural scaling for 3D NSE,

$$
u(x, t) \mapsto u_{\lambda}(x, t):=\frac{1}{\lambda} u_{\lambda}\left(\frac{x}{\lambda}, \frac{t}{\lambda^{2}}\right),
$$

we see that the a priori controlled quantities exhibit supercritical scaling - our examples scale as $\lambda^{1}$ - while those sufficient for regularity scale critically as $\lambda^{0}$. This mismatch is referred to as the "scaling-gap" and indicates 3D NSE is a supercritical problem.

Typically, conditional regularity results include premises which explicitly bridge this scaling gap. For example, a significant result in 
regularity theory was non-existence of solutions which are backwardin-time self-similar and singular (cf. [68, 88, 105]). Because self-similar solutions are by construction scaling invariant, their study is effectively a restriction to a class of critical solutions. Indeed, self-similar solutions satisfy a scaling invariant point-wise bound, ess sup $\left(\left(\left|x-x_{0}\right|+\right.\right.$ $\sqrt{T-t})|u(x, t)|)<\infty$, where the essential supremum is taken over an appropriate parabolic cylinder. For non-self-similar solutions satisfying this critical estimate, regularity has been affirmed in the axisymmetric case (cf. [25, 24, 74, 95]; the general case remains open). This discussion illustrates a general theme wherein, first, the flow is assumed to exhibit some feature which is critical and, second, additional restrictions are identified from which regularity is derived. Other criteria which possess this structure, for example the partial regularity result of [20], require additional conditions on the smallness of the presumed finite scale-invariant quantity.

In [43] and [61], a dynamic approach to bridging the scaling gap is introduced which is physically motivated by the persistence of the axial lengths of vortex filaments. In conjunction with known a priori decay rate for the volume of the region of intense vorticity (these can be obtained via results in [31] and [81]), this determines a decay rate for the axially-perpendicular radius of vortex filaments which matches the critical scaling of local, one-dimensional sparseness, a rate identified in [61] in terms of the decay of the uniform analyticity radius. Thus, the anisotropic physical picture indicates certain supercritical quantities behave critically in an asymptotic sense (it is in this regard that the scaling gap is bridged).

On its own the criticality discussed in [43] and [61] is insufficient to trigger the regularity criteria of [61] and additional work is required to break criticality. In Chapter 4 two strategies are given which break the critical scenario highlighted in [43] by establishing conditions which lead to logarithmic improvements to the known a priori decay rate of the volume of a localized super-level set of $|\omega|$ (these results are also contained in [17] and [18]). The decay rate is obtained as a consequence of the uniform-in-time control of the $L^{1}$ norm of the vorticity which is granted provided $\nabla \times u_{0}$ is a finite measure (cf. [31]). The logarithmic improvements we seek rely on establishing conditions which lead to the uniform-in-time control of local $L \log L$-type quantities.

Our two strategies are, in a sense, complimentary. In contrast to 
one another, the first class of conditions can be characterized as 'wild in time' possessing a uniform algebraic structure, while the second are 'wild in space' possessing a uniform geometric structure. The proofs are based on an adaptation of a method in [31], the novel component being the utilization of analytic cancellations in the vortex-stretching term via the Hardy space version of the Div-Curl Lemma [29], $\mathcal{H}^{1-}$ $B M O$ duality [103], and the intimate connection between the $B M O$ norm and the logarithm. The arguments diverge at a technical level. The key step for the former relies on the structure of the evolution of the scalar components of vorticity and a result from [102] which state that the $B M O$ norm of the logarithm of a polynomial is bounded independently of the coefficients. The latter approach depends on a sharp pointwise multiplier theorem in BMO [71] and Coifman-Rochberg's $B M O$-estimate on the logarithm of the maximal function of a locally integrable function [30] which depends only on the dimension of the space and, importantly, is independent of the function. 


\section{Chapter 2}

\section{Cascades and transport processes in astronomical and geophysical turbulence}

\section{$2.1 \quad$ Introduction}

Turbulent flow can be characterized by its high degree of spatial complexity. In the 3D hydrodynamic case, this is visible as the preferential distribution of energy on intermittent vortexes. As inertial mechanisms manipulate the flow, a dynamic process occurs in which energy is transported from larger to smaller scale structures (this motivates the term "cascade"). The foundational literature on this subject focuses on the hydrodynamic case, cf. [55] for an overview, and considerable mathematical effort has been applied to rigorously understand this process. Similar turbulent dynamics are apparent in more refined evolutionary systems. This chapter considers turbulent transport phenomena in the magnetohydrodynamical system and the surface quasi-geostrophic model. Here, we are most interested in affirming the existence of turbulent cascades across physically meaningful ranges of scales as well as showing that the inter-scale energy flux is local in the sense that it occurs predominantly between structures of comparable scale. Before attending these subjects we take a moment to more carefully introduce the idea of the turbulent cascade and briefly highlight prior developments relating to hydrodynamic turbulent cascades and flux locality.

A direct cascade, whether it be in a fluid or plasma, is the net inertial transport of an ideally conserved quantity from larger to smaller 
scales (cf. [28, 55, 8]). Roughly put, a source injects energy and, as the medium transitions to turbulence, large scale coherent structures emerge - vortex filaments in the hydrodynamic case; current sheets in 3D MHD turbulence - on which this energy is concentrated. As turbulence evolves, the energy is transported by inertial effects from macro-scale eddies to progressively smaller scales in a uni-directional fashion. The process ceases at a scale at which inertial effects are outweighed by dissipative forces (either viscous or resistive) and, instead of being transported to even smaller scales, energy is lost as heat. The range of scales over which this cascade persists is referred to as the inertial range. In the 3D hydrodynamic energy cascade, the inter-scale transport obeys two fundamental properties: constancy and scale locality of the flux. The first of these means that the energy flux at any particular scale within the inertial range is comparable to that at any other scale in the inertial range, i.e. at scales within the inertial range it is nearly constant. Locality of the flux means that the energy exchange is predominantly between structures of comparable scale.

A pioneering mathematical step toward rigorously affirming the existence of an energy cascade was carried out in frequency space by Foias, Manley, Rosa, and Temam in [52] (see also their monograph [53]). Regarding locality of the flux, positive steps were taken in the context of the Littlewood-Paley spectrum in [26] and [27] (see also [47, 98]). The creation and dynamics of vortex structures are relevant to this subject as their morphological properties relate to the spatial distribution of the vorticity and is connected to the regions on which the cascade is effected (see [31, 32, 38]).

A more recent approach has by R. Dascaliuc and Z. Grujić provides the foundation for our own efforts. In [40] they develop a dynamic, multi-scale averaging process which is applied to study features of viscous hydrodynamic turbulence (we recall the specifics of this methodology in Section 2.2.1; this technique has subsequently been used in $[41,42,43,45])$. The process acts as a detector of significant signfluctuations associated with a physical density at a given scale and is used to show that the orientation of a particular flux - i.e. the energy flux - is, in a statistically significant sense, is concentrative. The analysis is carried out entirely in physical space, a contrast to other approaches which have identified 'scale' with Fourier wavenumbers or Littlewood-Paley dyadic blocks. An interesting feature of this approach 
is that locality can be derived dynamically as a direct consequence of the existence of the turbulent cascade in view and exhibits comparable upper and lower bounds throughout the inertial range. In comparison, the previous locality results were essentially localized kinematic upper bounds on the flux, the corresponding lower bounds being consistent with turbulent properties of the flow [26, 47].

In this chapter we extend and adapt the approach of R. Dascaliuc and Z. Grujić to study turbulent transport in astronomical plasmas in Sections 2.2 and 2.3 as well as certain geophysical fluids in Section 2.4.

\section{$2.2 \quad$ Energy cascades and related processes in astronomical plasmas}

The 3D magnetohydrodynamic equations model the evolution of a coupled system comprised of a magnetic field and an electrically conducting fluid's velocity field. Throughout turbulent MHD regimes such as stellar winds and the interstellar medium, observational and numerical evidence indicate that energy is transported from larger to smaller scale structures in a regular fashion reminiscent of the hydrodynamic energy cascade (cf. [8, 12, 93, 9]). This process is consistent with the picture wherein energy is preferentially distributed on intermittently located and progressively thinning coherent current and vortex structures referred to as current sheets (cf. [8, 16, 60, 59, 19, 94]).

Although the existence of an energy cascade in 3D MHD turbulence is widely accepted in the physics community (see [8] for an overview and $[75,69]$ for the classical phenomenologies), there is considerable disagreement regarding the details. A contentious issue lies in understanding the anisotropic influence of a strong magnetic field on scaling properties of the energy spectrum (this discussion began in earnest in $[57,58]$; in contrast, the classical phenomenologies of Iroshnikov and Kraichnan assumed an isotropic spectral transfer [75, 69]). In [58], a critical balance assumption - i.e. that there is a single timescale for parallel and perpendicular motion (to the magnetic mean field) in a turbulent eddy - was introduced allowing for the derivations of a distinct perpendicular energy spectrum and a scaling relationship between the lengths of perpendicular and parallel fluctuations. Numerical results indicated the picture is more complex than that described in 
[58] and various competing phenomenologies have been developed (cf. $[12,86,56,7]$ for several examples). Lively debate remains as to which is the most effective (cf. $[91,6]$ ).

There is potential, in light of the discussion highlighted above, for a contribution to this debate based on a mathematical analysis of the governing system. Prior to now, however, neither the existence of an energy cascade nor the scale locality of the flux have been rigorously affirmed (non-rigorous results concerning locality of the flux can be found in [3]; a rigorous study of a related phenomenon, the concentration of enstrophy, has been carried out in [16] and is described in Section 3 of this chapter). The purpose of this section is to provide such results; in Section 2.2.3, we establish the cascade of total energy by studying the orientation of the total energy flux in a suitably statistical manner (which is detailed in Section 2.2.1) across an inertial range and, in Section 2.2.6, affirm scale locality of the energy flux.

We remark that our conclusions follow directly from the 3D MHD equations under assumptions which are physically reasonable for turbulent regimes wherein the magnetic Prandtl number is not significantly smaller than one (i.e. $\eta \lesssim \nu$ ). In particular, it applies to astronomical settings such as the Solar wind and the interstellar medium. Interestingly, and contrasting the current phenomenological theories cited earlier, no appeal is made to the existence of a strong magnetic guide field. This indicates that the energy cascade is an intrinsic property of the 3D MHD system and not solely an artifact of the plasma's environment.

Due to the coupling between the magnetic field and plasma, each of which is imbued with its own energy, there are a number of transfer mechanisms by which energy can 'flow' between scales. In particular, kinetic energy can remain tied to the velocity field or magnetic energy to the magnetic field, but each energy can also be transferred between the two fields. A secondary purpose of this section is to identify conditions under which the distinct energies are transported, in a statistical sense, from larger to smaller scales (see Sections 2.2.4 and 2.2.5). Additionally, by considering the stretching effect of the velocity field on the magnetic field lines, we identify a scenario and range of scales for which the dominant inter-field energy exchange is directed from the velocity field to the magnetic field (see Section 2.2.7). 


\subsection{1 $\left(K_{1}, K_{2}\right)$-covers and ensemble averages}

The main purpose of this section is to describe how ensemble averaging with respect to $\left(K_{1}, K_{2}\right)$-covers of an integral domain $B\left(0, R_{0}\right)$ can be used to establish essential positivity of an a priori sign-varying density over a range of physical scales associated with the integral domain (cf. [40]). The application to turbulence lies in showing certain flux densities are directed, on average, into structures of a particular scale i.e. the cascade is uni-directional from larger to smaller scales - as well as the near-constancy of the averaged densities - i.e. the space-time averages over cover elements are all mutually comparable - across a range of scales, the inertial range.

The ensemble averages will be taken over collections of space-time averages of physical densities localized to cover elements of a particular type of covering - a so called $\left(K_{1}, K_{2}\right)$-cover - of the region of turbulent activity. For simplicity, this region will be taken as a ball of radius $R_{0}$ centered at the origin and, to reflect the turbulence literature, is henceforth referred to as the integral domain (also known as the macroscale domain). The time interval on which we localize is chosen for dimensional consistency to be of length $T$ for which,

$$
T \geq \frac{R_{0}^{2}}{\nu}
$$

The $\left(K_{1}, K_{2}\right)$-covers are now defined.

Definition 2.2.1. Let $K_{1}, K_{2} \in \mathbb{N}$ and $0 \leq R \leq R_{0}$. The cover of the integral domain $B\left(0, R_{0}\right)$ by the $n$ (open) balls, $\left\{B\left(x_{i}, R\right)\right\}_{i=1}^{n}$ is a $\left(K_{1}, K_{2}\right)$-cover at scale $R$ if,

$$
\left(\frac{R_{0}}{R}\right)^{3} \leq n \leq K_{1}\left(\frac{R_{0}}{R}\right)^{3}
$$

and, for any $x \in B\left(0, R_{0}\right), x$ is contained in at most $K_{2}$ cover elements.

For the remainder of this section, all covers are understood to be $\left(K_{1}, K_{2}\right)$-covers at scale $R$. The positive integers $K_{1}$ and $K_{2}$ represent the maximum allowed global and local multiplicities, respectively.

In order to localize a physical density to a cover element we incorporate certain refined cut-off functions. For a cover element centered at 
$x_{i}$, let $\phi_{i}(x, t)=\eta(t) \psi(x)$ where $\eta \in C^{\infty}(0, T)$ and $\psi \in C_{0}^{\infty}\left(\mathbb{R}^{3}\right)$ satisfy,

$$
0 \leq \eta \leq 1, \eta=0 \text { on }(0, T / 3), \eta=1 \text { on }(2 T / 3, T), \frac{\left|\partial_{t} \eta\right|}{\eta^{\delta}} \leq \frac{C_{0}}{T},
$$

and,

$$
0 \leq \psi \leq 1, \psi=1 \text { on } B\left(x_{i}, R\right), \frac{\left|\partial_{i} \psi\right|}{\psi^{\rho}} \leq \frac{C_{0}}{R}, \frac{\left|\partial_{i} \partial_{j} \psi\right|}{\psi^{2 \rho-1}} \leq \frac{C_{0}}{R^{2}},
$$

where $3 / 4<\delta, \rho<1$.

By $\phi_{0}$ we denote a refined cut-off function centered at $x=0$ localizing to the ball $B\left(0, R_{0}\right)$ (thus $\phi_{0}$ is the cut-off function for the integral domain and is supported on $B\left(0,2 R_{0}\right)$ ).

Comparisons will be necessary between averaged quantities localized to cover elements at certain scales $R<R_{0}$ and averaged quantities taken at the scale of the integral domain, $R_{0}$. To accommodate this we impose several additional conditions for points $x_{i}$ lying near the boundary of $B\left(0, R_{0}\right)$. If $B\left(x_{i}, R\right) \subset B\left(0, R_{0}\right)$ we assume $\psi \leq \psi_{0}$. Alternatively, when $B\left(x_{i}, R\right) \not \subset B\left(0, R_{0}\right)$, additional assumptions are in order. To specify these, let $l(x, y)$ denote the collection of points on the line through $x$ and $y$ and define the sets,

$$
\begin{aligned}
S_{0}= & B\left(x_{i}, R\right) \cap B\left(0, R_{0}\right), \\
S_{1}= & \left\{x: R_{0} \leq|x|<2 R_{0}, \emptyset \neq\left(l(x, 0) \cap \partial B\left(x_{i}, R\right) \cap B\left(0, R_{0}\right)^{c}\right)\right\}, \\
S_{2}= & \left(\left\{x: R_{0} \leq|x|<2 R_{0}, \emptyset \neq\left(l\left(x, x_{0}\right) \cap \partial B\left(x_{i}, 2 R\right) \cap B\left(x_{0}, R_{0}\right)^{c}\right)\right\}\right. \\
& \left.\cup B\left(x_{i}, 2 R\right)\right) \cap\left(S_{0} \cup S_{1}\right)^{c} .
\end{aligned}
$$

Then, our assumptions are that $\psi$ satisfies (2.4.3), $\psi=1$ on $S_{0}, \psi=\psi_{0}$ on $S_{1}$, and $\operatorname{supp} \psi=S_{2}$. The above conditions ensure that $\psi \leq \psi_{0}$ and that $\psi$ can be constructed to have an inwardly oriented gradient field.

These tools are employed to study properties of a physical density at a physical scale $R$ associated with the integral domain $B\left(0, R_{0}\right)$. To illustrate this, let $\theta$ be a physical density (e.g. a flux density) and define its localized space-time average on a cover element at scale $R$ around $x_{i}$ as,

$$
\tilde{\Theta}_{x_{i}, R}=\frac{1}{T} \int_{0}^{T} \frac{1}{R^{3}} \int_{B\left(x_{i}, 2 R\right)} \theta(x, t) \phi_{i}^{\delta}(x, t) d x d t,
$$


where $0<\delta \leq 1$. Let $\langle\Theta\rangle_{R}$ denote the ensemble average over localized averages associated with cover elements, i.e.,

$$
\langle\Theta\rangle_{R}=\frac{1}{n} \sum_{i=1}^{n} \tilde{\Theta}_{x_{i}, R}
$$

Examining the values obtained by ensemble averaging the local averages associated to a variety of covers at a fixed scale allows us to draw conclusions about the flux density $\theta$ at comparable and greater scales. For instance, stability (i.e. near constancy) across the set $\left\{\langle\Theta\rangle_{R}\right\}$ indicates that the sign of $\theta$ is essentially uniform at scales comparable to or greater than $R$. On the other hand, if the sign were not essentially uniform at scale $R$, particular covers could be arranged to enhance negative and positive regions and thus give a wide range of sign varying values in $\left\{\langle\Theta\rangle_{R}\right\}$. Our methodology, then, establishes the essential positivity of an a priori sign varying density $\theta$ at a scale $R$, by showing the positivity and near constancy of all elements of $\left\{\langle\Theta\rangle_{R}\right\}$.

An indispensable observation is that, if $\theta$ is an a priori non-negative density, then the ensemble averages taken at scales below the integral scale are all comparable to the integral scale space-time average. We make this notion precise in the following lemma.

Lemma 2.2.2. Let $f(x, t) \in L_{l o c}^{1}\left((0, T) \times \mathbb{R}^{3}\right)$ be non-negative. Let $\left\{x_{i}\right\}_{i=1}^{n}$ be centers of elements of $a\left(K_{1}, K_{2}\right)$-cover of $B\left(x_{0}, R_{0}\right)$ at scale $R<R_{0}$. Setting,

$$
F_{0}=\frac{1}{T} \int_{0}^{T} \frac{1}{R_{0}^{3}} \int f(x, t) \phi_{0}(x, t) d x d t,
$$

and,

$$
F_{x_{i}, R}=\frac{1}{T} \int_{0}^{T} \frac{1}{R^{3}} \int f(x, t) \phi_{x_{i}, R}(x, t) d x d t
$$

we have,

$$
\frac{1}{K_{1}} F_{0} \leq\langle F\rangle_{R} \leq K_{2} F_{0}
$$

Proof. Recalling that $\phi_{x_{i}, R} \leq \phi_{0}$ and the definition of $\left(K_{1}, K_{2}\right)$-covers 
we have that,

$$
\begin{aligned}
\langle F\rangle_{R} & =\frac{1}{T} \int_{0}^{T} \frac{1}{n R^{3}} \int f(x, t) \sum_{i=1}^{n} \phi_{x_{i}, R}(x, t) d x d t \\
& \leq \frac{1}{T} \int_{0}^{T} \frac{1}{R^{3}} \frac{R^{3}}{R_{0}^{3}} \int f(x, t) K_{2} \phi_{0}(x, t) d x d t=K_{2} F_{0}
\end{aligned}
$$

and,

$$
\begin{aligned}
\langle F\rangle_{R} & =\frac{1}{T} \int_{0}^{T} \frac{1}{n R^{3}} \int f(x, t) \sum_{i=1}^{n} \phi_{x_{i}, R}(x, t) d x d t \\
& \geq \frac{1}{T} \int_{0}^{T} \frac{1}{R^{3}} \frac{1}{K_{1}} \frac{R^{3}}{R_{0}^{3}} \int f(x, t) \phi_{0}(x, t) d x d t=\frac{1}{K_{1}} F_{0} .
\end{aligned}
$$

For additional discussion of $\left(K_{1}, K_{2}\right)$-covers and ensemble averages, including some computational illustrations of the process, see [45].

\subsubsection{D incompressible MHD equations}

Our mathematical setting is that of weak solutions to the 3D magnetohydrodynamic equations over $\mathbb{R}^{3}$ (cf. [96] for the essential theory). Define $\mathcal{V}=\left\{f \in L^{2}\left(\mathbb{R}^{3}\right): \nabla \cdot f=0\right\}$ (where the divergence free condition is in the sense of distributions) and let $V$ be the closure of $\mathcal{V}$ under the norm of the Sobolev space, $\left(H^{1}\left(\mathbb{R}^{3}\right)\right)^{3}$, and, $H$, the closure of $\mathcal{V}$ under the $L^{2}$ norm. By a solution to MHD we mean a weak (distributional) solution to the coupled system,

$$
\left\{\begin{array}{l}
u_{t}-\nu \Delta u+(u \cdot \nabla) u-(b \cdot \nabla) b+\nabla\left(p+|b|^{2} / 2\right)=0 \\
b_{t}-\eta \Delta b+(u \cdot \nabla) b-(b \cdot \nabla) u=0 \\
\nabla \cdot u=\nabla \cdot b=0 \\
u(x, 0)=u_{0}(x) \in V \\
b(x, 0)=b_{0}(x) \in V
\end{array}\right.
$$

where $\eta$ and $\nu$ are the magnetic resistivity and kinematic viscosity respectively and $p(x, t)$ is the fluid pressure. 
Our present work utilizes suitable weak solutions for MHD. These are weak solutions which additionally satisfy the generalized energy inequality (among other things - see [66] for a precise definition),

$$
\begin{aligned}
& \int_{0}^{T} \int\left(\nu|\nabla u(x, t)|^{2}+\eta|\nabla b(x, t)|^{2}\right) \phi(x, t) d x d t \\
& \leq \frac{1}{2} \int_{0}^{T} \int\left(|u(x, t)|^{2}+|b(x, t)|^{2}\right) \phi_{t}(x, t) d x d t \\
& \quad+\frac{1}{2} \int_{0}^{T} \int\left(\nu|u(x, t)|^{2}+\eta|b(x, t)|^{2}\right) \Delta \phi(x, t) d x d t \\
& \quad+\frac{1}{2} \int_{0}^{T} \int\left(|u(x, t)|^{2}+|b(x, t)|^{2}+2 p(x, t)\right)(u(x, t) \cdot \nabla \phi(x, t)) d x d t \\
& \quad-\int_{0}^{T} \int(u(x, t) \cdot b(x, t))(b(x, t) \cdot \nabla \phi(x, t)) d x d t
\end{aligned}
$$

for a.e. $T \in(0, \infty)$ and any non-negative $\phi \in C_{0}^{\infty}\left(\mathbb{R}^{3} \times[0, \infty)\right)$.

Existence of suitable weak solutions for MHD is proven in [66] using an adaptation of the traditional method for NSE found in [20]. Our application of these will require the generalized energy inequality mentioned above as well as the following regularity properties (these can also be found in [66]).

Proposition 2.2.3. For $u_{0}, b_{0} \in H$ and $u_{0} \in W^{4 / 5,5 / 3}$, suppose $(u, b, p)$ constitutes a suitable weak solution to MHD. Then, $(u, b, p)$ satisfies,

$$
\begin{array}{r}
u, b \in L^{\infty}(0, \infty ; H), u, b \in L^{3}\left(0, \infty ; L^{3}\left(\mathbb{R}^{3}\right)\right) \\
\nabla u, \nabla b \in L^{2}\left(0, \infty ; L^{2}\left(\mathbb{R}^{3}\right)\right), p \in L^{3 / 2}\left(0, \infty ; L^{3 / 2}\left(\mathbb{R}^{3}\right)\right) .
\end{array}
$$

The fact that suitable weak solutions only satisfy a generalized energy inequality (as opposed to equality) introduces the possibility that energy is dissipated not only by viscosity or resistivity but also by singularities. In the case that the weak solution in question is regular, equality is attained in the generalized energy inequality, (2.2.4), and the potential for loss of flux due to singularities is eliminated. To streamline discussion we establish cascades for regular solutions and include an illustrative result for the non-regular case only in the context of the cascade of the modified (due to energy loss from possible singularities) total energy flux (cf. Section 2.2.3). The study of the possible energy 
loss due to singularities is itself an interesting subject but the case of MHD is not sufficiently distinct from that of NSE (which can be found in [40]) to justify an independent exposition.

\subsubsection{Total energy cascade}

The total energy flux through the boundary of the ball $B$ over the interval $(0, T)$ is given (cf. [8]) by,

$$
\frac{1}{2} \int_{0}^{T} \int_{\partial B}\left(|u|^{2}+|b|^{2}+2 p\right) \hat{n} \cdot u d x d t-\int_{0}^{T} \int_{\partial B}(u \cdot b)(\hat{n} \cdot b) d x d t
$$

where $\hat{n}$ is the unit normal vector directed inward. Our analytic results are enabled by substituting an inwardly directed vector field, $\nabla \phi_{i}$, for $\hat{n}$ where $\phi$ is a refined cut-off function for the ball $B\left(x_{i}, R\right)$. Localized, space-time averaged total energy fluxes into balls centered at the points $x_{i}$ of radius $R$, over the interval $(0, T)$, are then defined as,

$$
\begin{aligned}
F_{x_{i}, R}^{E}:= & \frac{1}{2} \int_{0}^{T} \int\left(|u|^{2}+|b|^{2}+2 p\right)\left(u \cdot \nabla \phi_{i}\right) d x d t \\
& -\int_{0}^{T} \int(u \cdot b)\left(b \cdot \nabla \phi_{i}\right) d x d t .
\end{aligned}
$$

It is informative to remark on the genesis of the last term, the advection of the cross-helicity via the magnetic field. The transfer of magnetic to kinetic energy is driven by the Lorentz force while the stretching of the magnetic field lines is responsible for the transfer of kinetic energy to magnetic energy. Since these are complementary, the sum experiences a global cancellation but locally leaves us with a flux-type term,

$$
\begin{aligned}
& \int_{0}^{T} \int((b \cdot \nabla) b \cdot \phi u+(b \cdot \nabla) u \cdot \phi b) d x d t \\
& =-\int_{0}^{T} \int(u \cdot b)(b \cdot \nabla \phi) d x d t .
\end{aligned}
$$

The appearance of this term is interesting because it is only non-zero if there is some degree of non-locality in the energy transfer between the two fields.

In the following we work in a fixed integral domain, $B\left(0, R_{0}\right)$, with associated cut-off $\phi_{0}$. Certain integral domain quantities will be used to 
determine lower bounds on the inertial ranges over which our cascades are shown to persist. These are the integral scale space-time averaged kinetic and magnetic energies and are defined in terms of a technical parameter, $\delta$, as,

$$
e_{0}^{u}=e_{0}^{u}(\delta)=\frac{1}{T} \int_{0}^{T} \frac{1}{R_{0}^{3}} \int \frac{1}{2}|u|^{2} \phi_{0}^{\delta} d x d t,
$$

and,

$$
e_{0}^{b}=e_{0}^{b}(\delta)=\frac{1}{T} \int_{0}^{T} \frac{1}{R_{0}^{3}} \int \frac{1}{2}|b|^{2} \phi_{0}^{\delta} d x d t,
$$

and the integral scale space-time averaged enstrophies which are given by,

$$
E_{0}^{u}=\nu \frac{1}{T} \int_{0}^{T} \frac{1}{R_{0}^{3}} \int|\nabla u|^{2} \phi_{0} d x d t
$$

and,

$$
E_{0}^{b}=\eta \frac{1}{T} \int_{0}^{T} \frac{1}{R_{0}^{3}} \int|\nabla b|^{2} \phi_{0} d x d t .
$$

The combined kinetic and magnetic energies or enstrophies will be identified by omitting the superscript (i.e. $e_{0}:=e_{0}^{u}+e_{0}^{b}$ ). Note that, because $e_{0}$ is decreasing with $\delta$, we will take liberties suppressing the dependence of $e_{0}$ on $\delta$ with the understanding that the indicated quantity is that associated with the smallest appropriate value.

We will establish that the cascade persists over a range of scales bound above by the integral scale and below by a modified Taylor micro-scale. The Taylor micro-scale is,

$$
\tau=\left(\frac{\nu e_{0}}{E_{0}}\right)^{1 / 2}
$$

and is taken from the hydrodynamic theory where, as a measure of inertial to viscous forces, it provides an intermediate scale between the macro-scale and the Kolmogorov dissipation scale (cf. [8]). Our modification will depend in part on the magnetic Prandtl number, denoted by 
$\operatorname{Pr}$, a non-dimensional number given by the ratio of kinematic viscosity to magnetic resistivity, i.e.,

$$
\operatorname{Pr}=\frac{\nu}{\eta}
$$

Essentially, its role in the modification is to incorporate information about the magnetic resistivity which is absent from our prescribed time scale (recall $T>R_{0}^{2} / \nu$ ) and the Taylor micro-scale.

With these definitions and labels in mind we are ready to present our main result regarding the existence of an inertial range combined energy cascade as an intrinsic feature of the 3D MHD system.

Theorem 2.2.4. Assume $u$ and $b$ are suitable weak solutions to $3 D$ MHD with sufficient regularity that equality holds in (2.2.4).

Let $\left\{x_{i}\right\}_{i=1}^{n} \subset B\left(0, R_{0}\right)$ be the centers of a $\left(K_{1}, K_{2}\right)$-cover at scale $R$. For a scale and cover independent positive parameter,

$$
\beta:=\left(\frac{1}{2 C K_{1} K_{2}\left(1+P r^{-1}\right)}\right)^{1 / 2}
$$

where $C$ is a constant determined by structural properties of $3 D \mathrm{MHD}$ and our cut-off functions, if $\tau / \beta<R_{0}$ then,

$$
\frac{1}{2 K_{1}} E_{0} \leq\left\langle F^{E}\right\rangle_{R} \leq 2 K_{2} E_{0}
$$

provided $R$ is contained in the interval $\left[\tau / \beta, R_{0}\right]$.

Proof. Let $\phi_{x_{i}, R}$ denote a refined cut-off function for the cover element $B\left(x_{i}, R\right)$. Assuming the premises above and in virtue of (2.2.4), we have for any cover element that,

$$
\begin{aligned}
F_{x_{i}, R}^{E} \geq & \int_{0}^{T} \int\left(\nu|\nabla u|^{2}+\eta|\nabla b|^{2}\right) \phi_{x_{i}, R} d x d t \\
& -\left|\frac{1}{2} \int_{0}^{T} \int\left(|u|^{2}+|b|^{2}\right) \partial_{t} \phi_{x_{i}, R} d x d t\right| \\
& +\left|\frac{1}{2} \int_{0}^{T} \int\left(\nu|u|^{2}+\eta|b|^{2}\right) \Delta \phi_{x_{i}, R} d x d t\right|
\end{aligned}
$$


Recalling the properties of our cut-off functions,

$$
\left|\partial_{t} \phi_{x_{i}, R}\right| \leq c_{0} \frac{\phi_{x_{i}, R}^{\rho}}{T}
$$

as well as the fact,

$$
\frac{1}{T} \leq \frac{\nu}{R^{2}}=\operatorname{Pr} \frac{\eta}{R^{2}}
$$

we conclude that,

$$
\begin{aligned}
& \frac{1}{2} \int_{0}^{T} \int\left(|u|^{2}+|b|^{2}\right) \partial_{t} \phi_{x_{i}, R} d x d t \\
& \leq c_{0} \frac{\nu}{R^{2}} \int_{0}^{T} \int|u|^{2} \phi_{x_{i}, R}^{\rho} d x d t+c_{0} \operatorname{Pr} \frac{\eta}{R^{2}} \int_{0}^{T} \int|b|^{2} \phi_{x_{i}, R}^{\rho} d x d t .
\end{aligned}
$$

Our cut-off functions also satisfy,

$$
\left|\Delta \phi_{x_{i}, R}\right| \leq c_{0} \frac{\phi^{2 \rho-1}}{R^{2}}
$$

and, consequently,

$$
\begin{aligned}
& \frac{1}{2} \int_{0}^{T} \int\left(\nu|u|^{2}+\eta|b|^{2}\right) \Delta \phi_{x_{i}, R} d x d t \\
& \leq c_{0} \frac{\nu}{R^{2}} \int_{0}^{T} \int|u|^{2} \phi_{x_{i}, R}^{2 \rho-1} d x d t+c_{0} \frac{\eta}{R^{2}} \int_{0}^{T} \int|b|^{2} \phi_{x_{i}, R}^{2 \rho-1} d x d t .
\end{aligned}
$$

Noting that $\rho>2 \rho-1$ we have, upon combining the above estimates, that,

$$
\begin{aligned}
F_{x_{i}, R}^{E} \geq & \int_{0}^{T} \int\left(\nu|\nabla u|^{2}+\eta|\nabla b|^{2}\right) \phi_{x_{i}, R} d x d t \\
& -\frac{c_{0}}{R^{2}} \int_{0}^{T} \int\left(\nu|u|^{2}+\eta(1+\operatorname{Pr})|b|^{2}\right) \phi_{x_{i}, R}^{2 \rho-1} d x d t .
\end{aligned}
$$

Using Lemma 2.2.2, we observe that,

$$
K_{2} E_{0} \geq\left\langle\frac{1}{T} \int_{0}^{T} \frac{1}{R^{3}} \int\left(\nu|\nabla u|^{2}+\eta|\nabla b|^{2}\right) \phi_{x_{i}, R} d x d t\right\rangle_{R} \geq \frac{1}{K_{1}} E_{0}
$$


and,

$$
\begin{aligned}
& \left\langle\frac{1}{T} \int_{0}^{T} \frac{1}{R^{3}} \int \frac{1}{2}\left(\nu|u|^{2}+\eta(1+\operatorname{Pr})|b|^{2}\right) \phi_{x_{i}, R}^{2 \rho-1} d x d t\right\rangle_{R} \\
& \leq \nu K_{2} e_{0}^{u}+\eta(1+\operatorname{Pr}) K_{2} e_{0}^{b} \\
& \leq \nu K_{2}\left(1+\operatorname{Pr}^{-1}\right) e_{0} .
\end{aligned}
$$

We can thus interpolate the ensemble average between integral scale quantities as,

$$
\frac{1}{K_{1}} E_{0}-\nu \frac{c_{0} K_{2}\left(1+P r^{-1}\right)}{R^{2}} e_{0} \leq\left\langle F^{E}\right\rangle_{R} \leq K_{2} E_{0}+\nu \frac{c_{0} K_{2}\left(1+P r^{-1}\right)}{R^{2}} e_{0}
$$

It is worth remarking that the upper bound follows in virtue of the assumed regularity (i.e. equality in (2.2.4)) and this is the only place where this assumption is used. In particular, the lower bound holds for non-regular solutions.

Continuing, we now specify a value for $\beta$, the modification to the inertial range, to be,

$$
\beta=\left(\frac{1}{2 c_{0} K_{1} K_{2}\left(1+P r^{-1}\right)}\right)^{1 / 2} .
$$

Because $R$ lies in the inertial range - i.e. $\tau / \beta \leq R \leq R_{0}$ - we have,

$$
\nu \frac{c_{0} K_{2}\left(1+P r^{-1}\right)}{R^{2}} e_{0} \leq \frac{1}{2 K_{1}} E_{0}
$$

The final lower bound for the ensemble average is thus,

$$
\left\langle F^{E}\right\rangle_{R} \geq \frac{1}{2 K_{1}} E_{0}
$$

The upper bound follows trivially with our definition of $\beta$ and we conclude that,

$$
\frac{1}{2 K_{1}} E_{0} \leq\left\langle F^{E}\right\rangle_{R} \leq 2 K_{2} E_{0}
$$


We make two remarks before proceeding to other topics. First, the condition triggering the cascade is essentially a requirement that the gradients of the velocity and the magnetic fields are large (averaged, over the integral domain) with respect to the fields themselves; this will hold in the regions of high spatial complexity of the flow (the correction parameter $\beta$ depends on certain a priori bounded quantities; however, none of these involve gradients). Second, the dependence of the length of the inertial range on $\operatorname{Pr}$ has consequences for when the above result is most physically relevant. The correction to the Taylor micro-scale is minimized when $\operatorname{Pr}^{-1} \lesssim 1$, that is, when $\eta \lesssim \nu$. This is exactly the scenario for turbulent plasmas in astronomical settings such as the solar wind and the interstellar medium.

A brief discussion of how to obtain a result for possibly non-regular suitable weak solutions is appropriate. As noted in the proof, the assumption of regularity was only used in establishing the upper bound on the ensemble average and we presently address how this can be alternatively accommodated. The physical cause of a strict inequality in the generalized energy inequality is interpreted as the loss of energy due to possible singularities. This lost energy will be denoted by $F_{\phi}^{\infty}$ (or $F_{x_{i}, R}^{\infty}$ if $\phi$ is the cut-off for a $\left(K_{1}, K_{2}\right)$-cover element) and is defined as the value that "fills in" the inequality (2.2.4); i.e., it satisfies,

$$
\begin{aligned}
& \int_{0}^{T} \int\left(\nu|\nabla u|^{2}+\eta|\nabla b|^{2}\right) \phi d x d t+F_{\phi}^{\infty} \\
& =\frac{1}{2} \int_{0}^{T} \int\left(|u|^{2}+|b|^{2}\right) \phi_{t} d x d t+\frac{1}{2} \int_{0}^{T} \int\left(\nu|u|^{2}+\eta|b|^{2}\right) \Delta \phi d x d t \\
& \quad+\frac{1}{2} \int_{0}^{T} \int\left(|u|^{2}+2|b|^{2}+2 p\right)(u \cdot \nabla \phi) d x d t \\
& \quad-\int_{0}^{T} \int(u \cdot b)(b \cdot \nabla \phi) d x d t .
\end{aligned}
$$

To account for the possible strictness of the above inequality we replace the fluxes considered previously with fluxes modified to include the possible loss of energy due to singularities. For example, in the case of the total energy flux, we establish an interpolative bound on ensemble averages corresponding to the localized modified total energy fluxes,

$$
F_{\phi}^{E, \infty}=F_{\phi}^{E}-F_{\phi}^{\infty}
$$


Positivity and near-constancy results are then given in terms of the modified flux specified above. The cascade of total energy modified due to possible non-regularity is then given by the following theorem, the

proof of which is identical modulo a substitution of $F_{\phi}^{E, \infty}$ for $F_{\phi}^{E}$ to the proof of (2.2.4).

Theorem 2.2.5. Assume $u$ and $b$ constitute a suitable weak solutions to 3D MHD. Let $\left\{x_{i}\right\}_{i=1}^{n} \subset B\left(0, R_{0}\right)$ be the centers of a $\left(K_{1}, K_{2}\right)$-cover at scale $R$ where the cut-off functions are defined with $T \geq R^{2} / \nu$. For a scale- and cover-independent, positive parameter,

$$
\beta:=\left(\frac{1}{2 C K_{1} K_{2}\left(1+P r^{-1}\right)}\right)^{1 / 2},
$$

where $C$ is a constant determined by structural properties of $3 D \mathrm{MHD}$ and our cut-off functions, if $\tau<\beta R_{0}$, then,

$$
\frac{1}{2 K_{1}} E_{0} \leq\left\langle F^{E, \infty}\right\rangle_{R} \leq 2 K_{2} E_{0}
$$

provided $R$ is contained in the interval $\left[\tau / \beta, R_{0}\right]$.

\subsubsection{Cascade-like dynamics of the total fluid en- ergy}

Our attention is now turned to establishing conditions under which distinct, inertially transported quantities exhibit cascade-like dynamics. Note that our terminology is modified to reflect the physics literature wherein the term 'cascade' typically refers to an ideally conserved quantity. We first consider the inertially driven concentration of total fluid energy. The localized flux quantity of interest is,

$$
\begin{aligned}
& \frac{1}{2} \int_{0}^{T} \int\left(|u|^{2}+2 p\right)(u \cdot \nabla \phi) d x d t \\
& =-\int_{0}^{T} \int((u \cdot \nabla) u+p) \cdot(\phi u) d x d t .
\end{aligned}
$$

Remark 2.2.1. The total fluid energy flux consists of the kinetic energy flux and the pressure flux, the local and the non-local parts, respectively. However, the cascade-like results obtained in this section, paired with 
the scale-locality of the flux presented in Section 7, indicates that the dominant component (on average) is the local one, i.e., the kinetic energy flux. Alternatively, one can study the kinetic energy cascade on its own and try to interpolate the ensemble-averaged pressure flux between suitable integral-scale quantities. See Section 2.2.5.

Results are ultimately intended for the dimensional form of the equations (as was the case for the cascade of total energy) but it will be convenient to carry out estimates in a dimensionless setting. Before doing so we verify that a positive result in one context implies an analogous result in the other. Beginning with a dimensional problem where the integral domain has radius $R_{0}$ (the characteristic length scale) and $\nu$ is the kinematic viscosity (these will be our fundamental dimensions; they also determine the characteristic time scale $T=R_{0}^{2} \nu^{-1}$ ), nondimensionalization is achieved using the dimensionless variables and functions,

$$
x_{i}^{*}=\frac{x_{i}}{R_{0}}, t^{*}=\frac{t}{T}, u^{*}(x, t)=\frac{T}{R_{0}} u(x, t), \text { and } b^{*}(x, t)=\frac{T}{R_{0}} b(x, t) .
$$

We will establish the existence of dimensionless cascade-like behavior over an inertial range determined by the relationship,

$$
\left(\frac{R_{0}}{R}\right)^{\delta} \frac{e_{0}^{*}}{E_{0}^{*}}<\beta
$$

where,

$$
e_{0}^{*}=\int_{0}^{1} \int \frac{1}{2}\left(\left|u^{*}\right|^{2}+\left|b^{*}\right|^{2}\right) \phi_{0} d x^{*} d t^{*},
$$

and,

$$
E_{0}^{*}=\int_{0}^{1} \int\left(\left|\nabla_{*} u^{*}\right|^{2}+\left|\nabla_{*} b^{*}\right|^{2}\right) \phi_{0} d x^{*} d t^{*},
$$

and $\beta$ is a dimensionless constant (here $\nabla_{*}$ indicates differentiation with respect to the dimensionless variable). This has consequences for the dimensional setting in virtue of the equivalence,

$$
\frac{e_{0}}{E_{0}}=R_{0}^{2} \frac{e_{0}^{*}}{E_{0}^{*}}
$$


which follows from a change of variable and the chain rule. Similar computations verify the following relationships,

$$
e_{0}^{u}=\frac{R_{0}^{2}}{T^{2}} e_{0}^{u^{*}}, e_{0}^{b}=\frac{R_{0}^{2}}{T^{2}} e_{0}^{b^{*}}, E_{0}^{u}=\frac{\nu}{T^{2}} E_{0}^{u^{*}}, \text { and } E_{0}^{b}=\frac{\eta}{T^{2}} E_{0}^{b^{*}},
$$

where the dimensionless quantities $e_{0}^{u^{*}}, e_{0}^{b^{*}}, E_{0}^{u^{*}}$, and $E_{0}^{b^{*}}$ are defined in analogy with their dimensional counterparts.

For the flux presently of interest, total (fluid) energy, setting,

$$
F_{x_{i}, R}=\frac{1}{T R^{3}} \int_{0}^{T} \int((u \cdot \nabla) u+\nabla p) \cdot\left(\phi_{x_{i}, R} u\right) d x d t
$$

and,

$$
\begin{gathered}
F_{x_{i}, R}^{*}=\frac{R_{0}^{3}}{R^{3}} \int_{0}^{1} \int\left(\left(u^{*}\left(x^{*}, t^{*}\right) \cdot \nabla_{*}\right) u^{*}\left(x^{*}, t^{*}\right)+\nabla_{*} p^{*}\left(x^{*}, t^{*}\right)\right) \\
\cdot \phi_{x_{i}, R}\left(x^{*}, t^{*}\right) u^{*}\left(x^{*}, t^{*}\right) d x^{*} d t^{*},
\end{gathered}
$$

we see that,

$$
F_{x_{i}, R}=\frac{\nu}{T^{2}} F_{x_{i}, R}^{*}
$$

The equivalence of non-dimensional and dimensional cascades can be seen by considering an example theorem in the dimensionless context which asserts that, for certain dimensionless quantities $\beta_{u}$ and $\beta_{b}$, if,

$$
\frac{R_{0}}{\beta_{u}}\left(\frac{e_{0}^{u^{*}}}{E_{0}^{u^{*}}}\right)^{1 / 4}<R<R_{0} \text { and } \frac{R_{0}}{\beta_{b}}\left(\frac{e_{0}^{b^{*}}}{E_{0}^{b^{*}}}\right)^{1 / 4}<R<R_{0},
$$

then,

$$
\frac{1}{2 K_{*}} E_{0}^{*} \leq\left\langle F^{*}\right\rangle_{R / R_{0}} \leq 2 K_{*} E_{0}^{*}
$$

By the quantitative relations identified above, the consequence for the dimensional scenario is, if,

$$
\frac{R_{0}^{1 / 2}}{\beta_{u}}\left(\frac{\nu e_{0}^{u}}{E_{0}^{u}}\right)^{1 / 4}<R<R_{0} \quad \text { and } \quad \frac{R_{0}^{1 / 2}}{\beta_{b}}\left(\frac{\eta e_{0}^{b}}{E_{0}^{b}}\right)^{1 / 4}<R<R_{0}
$$

then,

$$
\frac{1}{2 K_{*}} \widetilde{E}_{0} \leq\langle F\rangle_{R} \leq 2 K_{*} \widetilde{E}_{0}
$$


where $\tilde{E}_{0}$ is determined at the scale of the integral domain - in particular, it is independent of the scale and choice of a $\left(K_{1}, K_{2}\right)$-cover by,

$$
\widetilde{E}_{0}=\left(E_{0}^{u}+\operatorname{Pr} E_{0}^{b}\right)
$$

Finally, note that in the dimensionless variables our refined cut-off functions localize spatially to balls of (dimensionless) radius $R / R_{0}$ for $0<R \leq R_{0}$ and temporally to the (dimensionless) interval [0,1] and, in analogy to properties (2.4.2) and (2.4.3), satisfy the following gradient estimates,

$$
\left|\nabla_{*} \phi\left(x^{*}, t^{*}\right)\right| \leq c_{0} \frac{R_{0}}{R} \phi^{\rho}\left(x^{*}, t^{*}\right)
$$

and,

$$
\phi_{t^{*}}\left(x^{*}, t^{*}\right) \mid \leq c_{0} \phi^{\rho}\left(x^{*}, t^{*}\right) .
$$

Since we can recover dimensional cascade-like behavior from the non-dimensional counterpart we are justified in considering only the latter. We subsequently suppress the asterisks used above to indicate non-dimensionality noting that, for the remainder of this section, we are working with dimensionless quantities.

Following [96], solutions to dimensionless 3D MHD satisfy,

$$
\left\{\begin{array}{l}
\partial_{t} u-\frac{1}{R e} \Delta u=-(u \cdot \nabla) u-\nabla p-S\left(\nabla \frac{|b|^{2}}{2}-(b \cdot \nabla) b\right), \\
\partial_{t} b-\frac{1}{R m} \Delta b=\nabla \times(b \times u), \\
\nabla \cdot u=\nabla \cdot b=0,
\end{array}\right.
$$

where $R e$ and $R m$ are the Reynolds and magnetic Reynolds numbers respectively and the non-dimensional number, $S$, is defined in terms of the Hartmann number, $M$, to be $S=M^{2} /(R e R m$ ) (note that the Hartmann number is a dimensionless quantity given by the ratio of the electromagnetic force to the viscous force). We will assume the initial datum are divergence free and belong to the typical energy spaces, i.e.,

$$
u(x, 0)=u_{0}(x), b(x, 0)=b_{0}(x) \in L^{2}\left(\mathbb{R}^{3}\right) .
$$

In order to obtain a local cancellation between coupled non-linear terms, we assume sufficient regularity so that the following derivation 
is justified. Taking the scalar product of the equation of motion by $\phi u$ and the induction equation by $S \phi b$, integrating, and rearranging we obtain a flux density for total (fluid) energy into the ball $B\left(x_{i}, R / R_{0}\right)$ (here $\phi$ denotes an appropriate refined cut-off function),

$$
\begin{aligned}
& \int_{0}^{1} \int\left(\frac{1}{2}|u|^{2}+p\right) \cdot(\nabla \phi \cdot u) d x d t \\
& =\frac{1}{R e} \int_{0}^{1} \int|\nabla u|^{2} \phi d x d t+\frac{S}{R m} \int_{0}^{1} \int|\nabla b|^{2} \phi d x d t \\
& \quad-S \int_{0}^{1} \int \nabla \phi \cdot(b \times(u \times b)) d x d t \\
& \quad-\frac{1}{2} \int_{0}^{1} \int|u|^{2} \phi_{t} d x d t-\frac{1}{2 R e} \int_{0}^{1} \int|u|^{2} \Delta \phi d x d t \\
& -\frac{S}{2} \int_{0}^{1} \int|b|^{2} \phi_{t} d x d t-\frac{S}{2 R m} \int_{0}^{1} \int|b|^{2} \Delta \phi d x d t .
\end{aligned}
$$

Bounds for the lower order terms on the right hand side follow. The last four terms are bounded in a manner similar to that seen in the proof of (2.2.4). Here, however, we acknowledge the change of variable and cite the properties of our refined cut-off functions, (2.2.7), as well as the chain rule, to obtain the following bounds:

$$
\begin{aligned}
& \left.\left.\left|\frac{1}{2} \int_{0}^{1} \int\right| u\right|^{2} \phi_{t} d x d t\left|\leq c_{0}\left(\frac{R_{0}}{R}\right)^{2} \int_{0}^{1} \int\right| u\right|^{2} \phi^{4 \rho-3} d x d t \\
& \left.\left.\left|\frac{1}{2 R e} \int_{0}^{1} \int\right| u\right|^{2} \Delta \phi d x d t\left|\leq c_{0} \frac{1}{R e}\left(\frac{R_{0}}{R}\right)^{2} \int_{0}^{1} \int\right| u\right|^{2} \phi^{4 \rho-3} d x d t \\
& \left.\left.\left|\frac{S}{2} \int_{0}^{1} \int\right| b\right|^{2} \phi_{t} d x d t\left|\leq c_{0} S\left(\frac{R_{0}}{R}\right)^{2} \int_{0}^{1} \int\right| b\right|^{2} \phi^{\rho} d x d t \\
& \left.\left.\left|\frac{S}{2 R m} \int_{0}^{1} \int\right| b\right|^{2} \Delta \phi d x d t\left|\leq c_{0} \frac{S}{R m}\left(\frac{R_{0}}{R}\right)^{2} \int_{0}^{1} \int\right| b\right|^{2} \phi^{4 \rho-3} d x d t
\end{aligned}
$$

Repeatedly using Hölder's inequality, the Gagliardo-Nirenberg inequality, and Young's inequality, yields the following bound for the non-linear 
term originating in the induction equation,

$$
\begin{aligned}
& \left|c_{0} S \frac{R_{0}}{R} \int_{0}^{1} \int \phi^{\rho}(b \times(u \times b)) d x d t\right| \\
& \leq c_{0} S \frac{R_{0}}{R} \int_{0}^{1} \int\left(|u|^{1 / 2} \phi^{\rho-3 / 4}\right)\left(|b|^{1 / 2}\right)\left(|u|^{1 / 2}|b|^{3 / 2} \phi^{3 / 4}\right) d x d t \\
& \leq c_{0} S \frac{R_{0}}{R} \int_{0}^{1}\left\|u \phi^{2 \rho-3 / 2}\right\|_{2}^{1 / 2}\|b\|_{2}^{1 / 2}\left\|\left(|u|^{1 / 2}|b|^{3 / 2} \phi^{3 / 4}\right)\right\|_{2} d t \\
& \leq c_{0} \frac{R_{0}}{R}\left(\frac{M^{1 / 2} R m^{1 / 2}}{R e^{1 / 4}}\right)\left(\frac{M^{3 / 2}}{R e^{3 / 4} R m^{3 / 2}}\right) \\
& \quad \cdot \int_{0}^{1}\left\|u \phi^{2 \rho-3 / 2}\right\|_{2}^{1 / 2}\|b\|_{2}^{1 / 2}\|u\|_{2}^{1 / 2}\left\|\nabla\left(b \phi^{1 / 2}\right)\right\|_{2}^{3 / 2} d t \\
& \leq c_{0} \frac{M^{2} R m^{2} R_{0}^{4}}{R e R^{4}}\left(\sup _{t}\|u\|_{2}^{2}\right)\left(\sup _{t}\|b\|_{2}^{2}\right) \int_{0}^{1}\left\|u \phi^{4 \rho-3}\right\|_{2}^{2} d t \\
& \quad+\frac{S}{4 R m} \int_{0}^{1}\left\|\nabla\left(b \phi^{1 / 2}\right)\right\|_{2}^{2} d t \\
& \leq c_{0}(M R m)^{2}\left(\sup _{t}\|u\|_{2}^{2} \sup _{t}\|b\|_{2}^{2}\right) \frac{1}{R e} \frac{R_{0}^{4}}{R^{4}} \int_{0}^{1}\left\|u \phi^{4 \rho-3}\right\|_{2}^{2} d t \\
& \quad+\frac{S}{4 R m} \int_{0}^{1}\left\|(\nabla b) \phi^{1 / 2}\right\|_{2}^{2} d t+\frac{c_{0} S}{R m} \frac{R_{0}^{4}}{R^{4}} \int_{0}^{1}\left\|b \phi^{4 \rho-3}\right\|_{2}^{2} d t .
\end{aligned}
$$

Taking ensemble averages and applying Lemma 2.2.2 where needed we introduce the quantities $e_{0}^{u}, e_{0}^{b}, E_{0}^{u}$, and $E_{0}^{b}$ to obtain,

$$
\begin{aligned}
\langle F\rangle_{R} \geq & \frac{1}{K_{1}} \frac{1}{R e} E_{0}^{u}+\frac{1}{K_{1}} \frac{S}{R m} E_{0}^{b}-c_{0} \frac{K_{2} S}{R m}(2+R m) \frac{R_{0}^{4}}{R^{4}} e_{0}^{b} \\
& -c_{0} \frac{K_{2}}{R e}\left(1+R e+(M R m)^{2}\left(\sup _{t}\|u\|_{2}^{2} \sup _{t}\|b\|_{2}^{2}\right)\right) \frac{R_{0}^{4}}{R^{4}} e_{0}^{u} \\
= & \frac{1}{K_{1}} \frac{1}{R e} E_{0}^{u}-C_{u} \frac{K_{2}}{R e} \frac{R_{0}^{4}}{R^{4}} e_{0}^{u}+\frac{1}{K_{1}} \frac{S}{R m} E_{0}^{b}-C_{b} \frac{K_{2} S}{R m} \frac{R_{0}^{4}}{R^{4}} e_{0}^{b},
\end{aligned}
$$

where in the last line we have set,

$$
C_{u}=c_{0}\left(1+R e+(M R m)^{2}\left(\sup _{t}\|u\|_{2}^{2} \sup _{t}\|b\|_{2}^{2}\right)\right)
$$

and,

$$
C_{b}=c_{0}(2+R m)
$$


Similarly, an upper bound is,

$$
\begin{aligned}
\langle F\rangle_{R} \leq & K_{2} \frac{1}{R e} E_{0}^{u}+C_{u} K_{2} \frac{1}{R e} \frac{R_{0}^{4}}{R^{4}} e_{0}^{u} \\
& +K_{2} \frac{S}{R m} E_{0}^{b}+C_{b} K_{2} \frac{S}{R m} \frac{R_{0}^{4}}{R^{4}} e_{0}^{b}
\end{aligned}
$$

Defining now the parameters for a correction to the inertial range by,

$$
\beta_{u}=\left(\frac{1}{2 K_{1} K_{2} C_{u}}\right)^{1 / 4}
$$

and,

$$
\beta_{b}=\left(\frac{1}{2 K_{1} K_{2} C_{b}}\right)^{1 / 4}
$$

we have justified the following theorem.

Theorem 2.2.6. Let $\left\{x_{i}\right\}_{i=1}^{n} \subset B\left(0, R_{0}\right)$ be the centers of a $\left(K_{1}, K_{2}\right)$ cover at scale $R$. For $\beta_{u}$ and $\beta_{b}$ defined above, if,

$$
\tau:=\max \left\{\left(\frac{e_{0}^{u}}{E_{0}^{u}}\right)^{1 / 4},\left(\frac{e_{0}^{b}}{E_{0}^{b}}\right)^{1 / 4}\right\}<\min \left\{\beta_{u}, \beta_{b}\right\}=: \beta,
$$

then for scales $R$ where $\tau / \beta \leq R / R_{0}$, we have,

$$
\frac{1}{2 K_{1}}\left(\frac{1}{R e} E_{0}^{u}+\frac{S}{R m} E_{0}^{b}\right) \leq\langle F\rangle_{R} \leq 2 K_{2}\left(\frac{1}{R e} E_{0}^{u}+\frac{S}{R m} E_{0}^{b}\right) .
$$

Remark 2.2.2. The above is particularly relevant in scenarios where $S / R m \sim 1$ and the integral scale magnetic energy dominates the integral scale kinetic energy. In this case it is possible to free the parameter $\beta_{u}$ from its dependence on Re by replacing (2.2.8) with the bound,

$$
\frac{1}{2} \int_{0}^{1} \int|u|^{2} \partial_{t} \phi_{x_{i}, R} d x d t \leq K_{2} e_{0}^{b}
$$

This accommodates large values of Re and is applicable to settings involving the confinement of a liquid metal by a strong magnetic guide field [106]. Without these assumptions on the flow the physical relevance of the above result is diminished by the fact that the dependencies of $\beta_{u}$ and $\beta_{b}$ on the fluid and magnetic Reynold's numbers result in an inertial range which decreases in length as $R m$ and Re increase. 


\subsubsection{Other cascade-like dynamics}

We presently identify conditions under which cascade-like dynamics are exhibited by individual energy exchange mechanisms such as the exchange of energy between the velocity and magnetic fields. We continue to work with the dimensionless formulation and note that throughout this section all solutions are assumed to be suitable weak solutions to the dimensionless 3D MHD system which are regular enough for the localized energy equality to hold.

The localized (by the scalar function $\phi$ to a ball of radius $R / R_{0}$ ) flux term responsible for the $u$-to- $u$ energy exchange driven by the advection of the velocity field is,

$$
\begin{aligned}
F_{x_{i}, R}^{u} & :=-\int_{0}^{1} \int(u \cdot \nabla) u \cdot(\phi u) d x d t \\
& =\frac{1}{2} \int_{0}^{1} \int|u|^{2}(u \cdot \nabla \phi) d x d t .
\end{aligned}
$$

Similarly, the (localized) $b$-to- $b$ energy transfer driven by the advection of the velocity field is,

$$
\begin{aligned}
F_{x_{i}, R}^{b} & :=-\int_{0}^{1} \int(u \cdot \nabla b) \cdot(\phi b) d x d t \\
& =\frac{1}{2} \int_{0}^{1} \int|b|^{2}(u \cdot \nabla \phi) d x d t .
\end{aligned}
$$

The fluid pressure flux-type term is,

$$
F_{x_{i}, R}^{p}:=-\int_{0}^{1} \int \nabla p \cdot(\phi u) d x d t=\int_{0}^{1} \int p(u \cdot \nabla \phi) d x d t .
$$

As already mentioned, the transfer of magnetic to kinetic energy is driven by the Lorentz force while the stretching of the magnetic field lines is responsible for the transfer of kinetic energy to magnetic energy; combined (locally), they yield the following term - the advection of cross-helicity by the magnetic field,

$$
\begin{aligned}
F_{x_{i}, R}^{u b} & :=\int_{0}^{1} \int((b \cdot \nabla) b \cdot(\phi u)+(b \cdot \nabla) u \cdot(\phi b)) d x d t \\
& =-\int_{0}^{1} \int(u \cdot b)(b \cdot \nabla \phi) d x d t .
\end{aligned}
$$


Until now we have only investigated cascades associated with collections of flux-type terms including the term for the flux of the fluid pressure. Due to the unique structure of this term, additional effort is required to establish cascade-like behavior for combined fluxes excluding the pressure flux. More precisely, we will need to bound the quantity,

$$
\int_{0}^{1} \int p(u \cdot \nabla \phi) d x d t
$$

To do so we will use the estimate,

$$
\begin{aligned}
\left\|u \phi^{1 / 2}\right\|_{3} & =\left(\int\left(|u|^{3 / 2} \phi^{3 / 4}\right)\left(|u|^{3 / 2} \phi^{3 / 4}\right) d x\right)^{1 / 3} \\
& \leq\left(\left(\int|u|^{2} \phi d x\right)^{3 / 4}\left(\int|u|^{6} \phi^{3} d x\right)^{1 / 4}\right)^{1 / 3} \\
& \leq C\left\|u \phi^{1 / 2}\right\|_{2}^{1 / 2}\left\|\nabla\left(u \phi^{1 / 2}\right)\right\|_{2}^{1 / 2},
\end{aligned}
$$


which leads to the estimate,

$$
\begin{aligned}
\left|\int_{0}^{1} \int p(u \cdot \nabla \phi) d x d t\right| & \\
\leq & C \frac{R_{0}}{R} \int_{0}^{1}\left\|p \phi^{\rho-1 / 2}\right\|_{3 / 2}\left\|u \phi^{1 / 2}\right\|_{3} d t \\
\leq & C \frac{R_{0}}{R} \int_{0}^{1}\left\|p \phi^{\rho-1 / 2}\right\|_{3 / 2}\left\|u \phi^{1 / 2}\right\|_{2}^{1 / 2}\left\|\nabla\left(u \phi^{1 / 2}\right)\right\|_{2}^{1 / 2} d t \\
\leq & C \frac{R_{0}}{R}\left(\int_{0}^{1}\left\|p \phi^{\rho-1 / 2}\right\|_{3 / 2}^{3 / 2} d t\right)^{2 / 3}\left(\int_{0}^{1}\left\|u \phi^{1 / 2}\right\|_{2}^{6} d t\right)^{1 / 12} \\
& \cdot\left(\int_{0}^{1}\left\|\nabla\left(u \phi^{1 / 2}\right)\right\|_{2}^{2} d t\right)^{1 / 4} \\
\leq & C R e^{1 / 3}\left(\frac{R_{0}}{R}\right)^{4 / 3}\left(\int_{0}^{T}\left\|p \phi^{\rho-1 / 2}\right\|_{3 / 2}^{3 / 2} d t\right)^{8 / 9}\left(\int_{0}^{1}\left\|u \phi^{1 / 2}\right\|_{2}^{6} d t\right)^{1 / 9} \\
& +\frac{1}{8 R e} \int_{0}^{1}\left\|\nabla\left(u \phi^{1 / 2}\right)\right\|_{2}^{2} d t \\
\leq & C_{p} R e^{1 / 3}\left(\frac{R_{0}}{R}\right)^{4 / 3}\left(\int_{0}^{1}\left\|u \phi^{1 / 2}\right\|_{2}^{2} d t\right)^{1 / 9} \\
& +C \frac{1}{R e}\left(\frac{R_{0}}{R}\right)^{2} \int_{0}^{1}\left\|u \phi^{2 \rho-1}\right\|_{2}^{2} d t \\
& +\frac{1}{4 R e} \int_{0}^{1}\left\|(\nabla u) \phi^{1 / 2}\right\|_{2}^{2} d t,
\end{aligned}
$$

where we have used Hölder's inequality, the Gagliardo-Nirenberg inequality, and Young's inequality. Note that the quantity appearing above,

$$
C_{p}=\left(\sup _{t}\left\|u \phi_{0}^{1 / 2}\right\|_{2}\right)^{4 / 9}\left(\int_{0}^{1}\left\|p \phi_{0}^{\rho-1 / 2}\right\|_{3 / 2}^{3 / 2} d t\right)^{8 / 9}
$$

is dimensionless and a priori bounded in virtue of regularity properties of suitable weak solutions (cf. [66]). In addition, it contains no gradients and is independent of the particular cover element at scale $R$.

Regarding the first term on the right hand side of (2.2.14), in order to pass from ensemble averages of localized quantities to an integral scale quantity we will need a simple consequence of the finite form 
of Jensen's inequality. Specifically, for a set of non-negative values, $\left\{a_{i}\right\}_{i=1}^{n}$, we have,

$$
\sum_{i=1}^{n} \frac{a_{i}^{1 / 9}}{n} \leq\left(\sum_{i=1}^{n} \frac{a_{i}}{n}\right)^{1 / 9} .
$$

Taking an ensemble average of normalized quantities yields,

$$
\begin{aligned}
& \frac{C_{p}}{n} \sum_{i=1}^{n}\left(\frac{R_{0}}{R}\right)^{3}\left(\frac{R_{0}}{R}\right)^{\frac{4}{3}}\left(\int_{0}^{1} \int|u|^{2} \phi_{x_{i}, R} d t\right)^{\frac{1}{9}} \\
& \leq C_{p}\left(\frac{R_{0}}{R}\right)^{4}\left(\frac{1}{n} \sum_{i=1}^{n} \int_{0}^{1}\left(\frac{R_{0}}{R}\right)^{3} \int|u|^{2} \phi_{x_{i}, R} d x d t\right)^{\frac{1}{9}} \\
& \leq C_{p}\left(\frac{R_{0}}{R}\right)^{4}\left(K_{2} e_{0}^{u}\right)^{\frac{1}{9}} .
\end{aligned}
$$

Bounds for the remaining flux densities are,

$$
\begin{aligned}
\left|F_{x_{i}, R}^{u}\right| \leq & C \frac{R_{0}}{R} \int_{0}^{1}\|u\|_{2}\left\|u \phi^{2 \rho-3 / 2}\right\|_{2}^{1 / 2}\left\|\nabla\left(u \phi^{1 / 2}\right)\right\|_{2}^{3 / 2} d x d t \\
\leq & C\left(\frac{R_{0}}{R}\right)^{4} \sup _{t}\|u\|_{2}^{4} \int_{0}^{1}\left\|u \phi^{2 \rho-3 / 2}\right\|_{2}^{2} d t \\
& +\frac{1}{8 R e} \int_{0}^{1}\left\|\nabla\left(u \phi^{1 / 2}\right)\right\|_{2}^{2} d t \\
\leq & C\left(R e^{4 / 3} \sup _{t}\|u\|_{2}^{4}+1\right) \frac{1}{R e}\left(\frac{R_{0}}{R}\right)^{4} \int_{0}^{1}\left\|u \phi^{2 \rho-3 / 2}\right\|_{2}^{2} d t \\
& +\frac{1}{4 R e} \int_{0}^{1}\left\|(\nabla u) \phi^{1 / 2}\right\|_{2}^{2} d t,
\end{aligned}
$$

and,

$$
\begin{aligned}
\left|F_{x_{i}, R}^{b}\right|,\left|F_{x_{i}, R}^{u b}\right| \leq & \frac{S}{4 R m} \int_{0}^{1}\left\|(\nabla b) \phi^{1 / 2}\right\|_{2}^{2} d t \\
+ & C\left(R m^{4 / 3} \sup _{t}\|u\|_{2}^{4}+1\right) \frac{S}{R m}\left(\frac{R_{0}}{R}\right)^{4} \\
& \cdot \int_{0}^{1}\left\|b \phi^{2 \rho-3 / 2}\right\|_{2}^{2} d t .
\end{aligned}
$$


Henceforth, we will be concerned with the scenario in which the kinetic energy over the integral domain and within the prescribed timescale remains bounded away from zero. In particular we take this to mean $e_{0}^{u} \geq 1$. This is the trade-off for considering purely kinetic fluxes. The result concerning cascade-like behavior for the direct kinetic to kinetic energy transfer driven by the advection of the velocity field follows.

Theorem 2.2.7. Assume $u$ and $b$ are solutions of 3D MHD possessing sufficient regularity so that the generalized energy equality holds. Let $\left\{x_{i}\right\}_{i=1}^{n} \subset B\left(0, R_{0}\right)$ be the centers of a $\left(K_{1}, K_{2}\right)$-cover at scale $R$. Suppose that the flow is such that $e_{0}^{u} \geq 1$. For certain values $\beta_{u}$ and $\beta_{b}$, if,

$$
\tau:=\max \left\{\left(\frac{e_{0}^{u}}{E_{0}^{u}}\right)^{1 / 4},\left(\frac{e_{0}^{b}}{E_{0}^{b}}\right)^{1 / 4}\right\}<\min \left\{\beta_{u}, \beta_{b}\right\}=: \beta,
$$

then, for scales $R$ where $\tau / \beta \leq R / R_{0}$, we have,

$$
\frac{1}{2 K_{1}}\left(\frac{1}{R e} E_{0}^{u}+\frac{S}{R m} E_{0}^{b}\right) \leq\left\langle F^{u}\right\rangle_{R} \leq 2 K_{2}\left(\frac{1}{R e} E_{0}^{u}+\frac{S}{R m} E_{0}^{b}\right) .
$$

Proof. The main estimates have already been established. Based on the localized energy equality we have

$$
\begin{aligned}
F_{x_{i}, R}^{u} \geq & \frac{1}{R e} E_{x_{i}, R}^{u}+\frac{S}{R m} E_{x_{i}, R}^{b}-\left|F_{x_{i}, R}^{p}+N_{x_{i}, R}\right| \\
& -\frac{1}{2} \int_{0}^{1} \int|u|^{2} \phi_{t} d x d t-\frac{1}{2 R e} \int_{0}^{1} \int|u|^{2} \Delta \phi d x d t \\
& -\frac{S}{2} \int_{0}^{1} \int|b|^{2} \phi_{t} d x d t-\frac{S}{2 R m} \int_{0}^{1} \int|b|^{2} \Delta \phi d x d t .
\end{aligned}
$$

By the assumption on the flow we can replace the exponent of $1 / 9$ in the bound (2.2.16) with 1 . Taking ensemble averages, we obtain the lower bound,

$$
\begin{aligned}
\left\langle F^{u}\right\rangle_{R} \geq & \frac{1}{2 K_{1} R e} E_{x_{i}, R}^{u}-C_{u} K_{2} \frac{1}{R e}\left(\frac{R_{0}}{R}\right)^{4} e_{0}^{u} \\
& +\frac{S}{K_{1} R m} E_{x_{i}, R}^{b}-C_{b} K_{2} \frac{S}{R m}\left(\frac{R_{0}}{R}\right)^{4} e_{0}^{b},
\end{aligned}
$$


where

$$
C_{u}=C\left(C_{p} R e^{4 / 3}+R e+1+(M R m)^{2} \sup _{t}\|u\|_{2}^{2} \sup _{t}\|b\|_{2}^{2}\right)
$$

and,

$$
C_{b}=C\left(R m^{4 / 3} \sup _{t}\|u\|_{2}^{4}+R m+1\right) .
$$

This is sufficient to establish values for $\beta_{u}$ and $\beta_{b}$ (containing no gradients) and conclude in the standard fashion.

At this point, noting that $F^{b}$ and $F^{u b}$ both satisfy (2.2.9), we have already demonstrated the steps involved in establishing cascade-like behavior for the densities $F^{b}$ and $F^{u b}$. We consequently omit the proofs.

Theorem 2.2.8. Let $\left\{x_{i}\right\}_{i=1}^{n} \subset B\left(0, R_{0}\right)$ be the centers of a $\left(K_{1}, K_{2}\right)$ cover at scale $R$. Suppose that the flow is such that $e_{0}^{u} \geq 1$. For certain values $\beta_{u}$ and $\beta_{b}$, if,

$$
\tau:=\max \left\{\left(\frac{e_{0}^{u}}{E_{0}^{u}}\right)^{1 / 4},\left(\frac{e_{0}^{b}}{E_{0}^{b}}\right)^{1 / 4}\right\}<\min \left\{\beta_{u}, \beta_{b}\right\}=: \beta,
$$

then for scales $R$ where $\tau / \beta \leq R / R_{0}$, we have,

$$
\frac{1}{2 K_{1}}\left(\frac{1}{R e} E_{0}^{u}+\frac{S}{R m} E_{0}^{b}\right) \leq\left\langle F^{b}, F^{u b}\right\rangle_{R} \leq 2 K_{2}\left(\frac{1}{R e} E_{0}^{u}+\frac{S}{R m} E_{0}^{b}\right)
$$

\subsubsection{Locality of the energy flux}

According to turbulence phenomenology in the purely hydrodynamical setting, the average energy flux at scale $R$ is supposed to be wellcorrelated only with the average fluxes at nearby scales (throughout the inertial range). This phenomenon has been confirmed in [47, 26, 40, 41]. In the plasma setting, the question of locality has been somewhat controversial. Recently, Aluie and Eyink [3] produced an argument in favor of locality of the total energy and cross-helicity fluxes. Numerical work also supports locality (cf. [46] for the case of decaying turbulence).

Our context allows us to affirm a particular flux's locality as a direct consequence of the existence of the corresponding, nearly-constant turbulent cascade per unit mass. We illustrate this in the case of the 
kinetic energy flux (transported by the velocity). Denote the timeaveraged local fluxes associated to the cover element $B\left(x_{i}, R\right)$ by $\hat{\Psi}_{x_{i}, R}$,

$$
\hat{\Psi}_{x_{i}, R}=\frac{1}{T} \int_{0}^{T} \int \frac{1}{2}|u|^{2}\left(u \cdot \nabla \phi_{i}\right) d x,
$$

and the time-averaged local fluxes associated to the cover element $B\left(x_{i}, R\right)$, per unit mass, by $\hat{\Phi}_{x_{i}, R}$,

$$
\hat{\Phi}_{x_{i}, R}=\frac{1}{T} \int_{0}^{T} \frac{1}{R^{3}} \int \frac{1}{2}|u|^{2}\left(u \cdot \nabla \phi_{i}\right) d x .
$$

Then, the (time and ensemble) averaged flux is given by,

$$
\langle\Psi\rangle_{R}=\frac{1}{n} \sum_{i=1}^{n} \hat{\Psi}_{x_{i}, R}=R^{3}\langle\Phi\rangle_{R}=R^{3} \frac{1}{n} \sum_{i=1}^{n} \hat{\Phi}_{x_{i}, R} .
$$

The following manifestation of locality follows directly from Theorem 2.2.7. Let $R$ and $r$ be two scales within the inertial range delineated in the theorem. Then,

$$
\frac{1}{4 K_{1}^{2}}\left(\frac{r}{R}\right)^{3} \leq \frac{\langle\Psi\rangle_{r}}{\langle\Psi\rangle_{R}} \leq 4 K_{2}^{2}\left(\frac{r}{R}\right)^{3} .
$$

In particular, if $r=2^{k} R$ for some integer $k$,

$$
\frac{1}{4 K_{1}^{2}} 2^{3 k} \leq \frac{\langle\Psi\rangle_{2^{k} R}}{\langle\Psi\rangle_{R}} \leq 4 K_{2}^{2} 2^{3 k},
$$

i.e., along the dyadic scale, the locality propagates exponentially.

\subsubsection{A scenario exhibiting predominant $u$-to- $b$ en- ergy transfer}

In [43] a dynamic estimate is given on the vortex-stretching term (in the vorticity formulation of 3D NSE) - across a range of scales - using the ensemble averaging process we have illustrated above. The purpose was to present a mathematical evidence of the creation and persistence of integral scale length vortex filaments by establishing the positivity of the ensemble-averaged vortex stretching term across a range of scales extending to the integral scale. 
In the induction equation for the magnetic field, the nonlinear term $(b \cdot \nabla) u$ is responsible for the stretching of magnetic field lines. Positivity of $(b \cdot \nabla) u \cdot(\phi b)$ indicates the magnetic field line is being elongated, a phenomenon which corresponds to a transfer of energy from the velocity field to the magnetic field (negativity would reflect a diminution of the field line and a local transport of energy from the magnetic field to the fluid flow). Consequently, to conclude that the predominant energy exchange between the velocity and magnetic fields is from the velocity field to the magnetic field across a range of physical scales, it will be sufficient to establish (in an appropriate statistical sense) the positivity of $(b \cdot \nabla) u \cdot(\phi b)$ across these scales. Before proceeding to this task we remark that recent numerical work (cf. [46]) indicates that imbalanced exchanges are common in certain forced and decaying turbulent regimes.

We label the space-time localized quantity of interest as,

$$
V_{x_{i}, R}=\int_{0}^{1} \int(b \cdot \nabla) u \cdot\left(b \phi_{x_{i}, R}\right) d x d t
$$

and note that our work is carried out in the context of the dimensionless formulation of 3D MHD where we take $R m=S=1$ for convenience. We also assume the weak solution in question is regular.

Theorem 2.2.9. Let $\left\{x_{i}\right\}_{i=1}^{n} \subset B\left(0, R_{0}\right)$ be the centers of a $\left(K_{1}, K_{2}\right)$ cover at scale $R$. For a certain value (which will be apparent in the proof) $\beta>0$, if,

$$
\tau:=\left(\frac{e_{0}^{b}}{E_{0}^{b}}\right)^{1 / 4}<\beta,
$$

then for scales $R$ where $\tau / \beta \leq R / R_{0}$, we have,

$$
\frac{1}{2 K_{1}} E_{0}^{b} \leq\left\langle V_{x_{i}, R}\right\rangle_{R} \leq 2 K_{2} E_{0}^{b} .
$$

Proof. Starting with the induction equation it is routine to obtain,

$$
\begin{aligned}
\int_{0}^{1} \int(b \cdot \nabla) u \cdot\left(b \phi_{x_{i}, R}\right) d x d t= & \int_{0}^{1} \int|\nabla b|^{2} \phi_{x_{i}, R} d x d t \\
& -\int_{0}^{1} \int \frac{1}{2}|b|^{2}\left(\partial_{t} \phi_{x_{i}, R}-\Delta \phi_{x_{i}, R}\right) d x d t \\
& +\frac{1}{2} \int_{0}^{1} \int|b|^{2}\left(u \cdot \nabla \phi_{x_{i}, R}\right) d x d t
\end{aligned}
$$


Note that, for a refined cut-off function $\phi$, the bounding process evident in the derivation of (2.2.9) can be modified to yield,

$$
\begin{aligned}
\int_{0}^{1} \int \frac{|b|^{2}}{2} u \cdot \nabla \phi d x d t \leq & C\left(\frac{R_{0}}{R}\right)^{4}\left(\sup _{t}\|u\|_{2}\right)^{4} \int_{0}^{1}\|b \phi\|_{2}^{2} d t \\
& +\frac{1}{4} \int_{0}^{1}\|\nabla(\phi b)\|_{2}^{2} d t
\end{aligned}
$$

Consequently, after taking ensemble averages and recalling the last two estimates in (2.2.8),

$$
\left\langle V_{x_{i}, R}\right\rangle_{R} \leq K_{2} E_{0}^{b}+K_{2}\left(\sup _{t}\|u\|_{2}^{4}+2\right)\left(\frac{R_{0}}{R}\right)^{2} e_{0}^{b}
$$

and,

$$
\left\langle V_{x_{i}, R}\right\rangle_{R} \geq K_{2} E_{0}^{b}-\frac{C}{2 K_{1}}\left(\sup _{t}\|u\|_{2}^{4}+2\right)\left(\frac{R_{0}}{R}\right)^{2} e_{0}^{b} .
$$

Selecting an appropriate value for $\beta$ allows us to conclude in the standard fashion.

\subsection{Enstrophy transport and concentra- tion in astronomical plasmas}

We have mentioned previously that observational and numerical data support a picture of turbulent MHD regimes comprised of intermittently distributed regions of high spatial complexity - these are the coherent current and vortex structures, i.e. current sheets - which become increasingly thin as turbulence evolves [8, 60, 59, 19, 94]. One view is that this process is initiated and driven by inertial effects associated with the cascades of ideally conserved quantities, (see [82, 48, 97, 110]; regarding the genesis of solar wind current sheets, an alternative view is given in $[19,13]$ where it is speculated that they are magnetic flux tubes generated in the solar corona and passively advected by the solar wind; modern theories and numerical studies regarding turbulent cascades and spectra can be found in $[9,12,15,93,90]$ and were briefly discussed previously in Chapter 2.2). 
Although enstrophy - taken here as the sum of the squares of vorticity and current - is not conserved in the ideal equations (and so we avoid the term "enstrophy cascade"), it does become increasingly concentrated on small-scale current sheets. Understanding this concentrative process is important because it effects the development of current-driven instabilities (for example, the tearing instability, cf. [8]) which drive magnetic reconnection (the relationship between magnetic reconnection and current structures has been studied in $[48,94])$.

This section is concerned with establishing conditions which rigorously affirm that the inertially driven transport of enstrophy is concentrative across an appropriate range of scales. In particular, we show the enstrophy flux is predominantly oriented (in a statistical sense) from larger to smaller scale structures and, moreover, is local and occurs at a nearly constant rate. In the case that non-inertial effects are negligible at large scales this indicates a detectable concentration of enstrophy where the inertial contribution is cascade-like. Also of note is the implication that, at least in our scenario, inertial forces - i.e. advection of the enstrophy by the fluid - effect the morphology of current sheets and, therefore, these structures are not passively advected by the fluid medium.

Although our main interest is the 3D setting, for illustrative purposes it is useful to consider the concentrative progression in $2 \mathrm{D}$ (we here summarize [8]; it is the intention of the author and collaborators to comment specifically on the $2 \mathrm{D}$ case in a future paper concerned with a variety of features of $2 \mathrm{D}$ MHD turbulence including the inverse cascade of magnetic potential and the direct cascade of energy). In 2D, early in the turbulent evolution, the current is predominantly distributed along eddy boundaries while, at later stages, it becomes spiked at eddy centers. Unlike 2D fluid turbulence where the concentration of enstrophy is driven solely by inertial forces - the enstrophy cascade - the Lorentz force does not vanish in 2D MHD and therefore introduces a possible source of enstrophy $[28,55,32,38]$. This complicates the creation, transport, and destruction of enstrophy and, consequently, something superficially similar to the fluid "enstrophy cascade" should only be visible if the Lorentz force is depleted by some intrinsic mechanism and the enstrophy flux is nearly constant, local, and directed from larger to smaller scales. Heuristic arguments and numerical evidence indicate that such depletive effects exist (cf. [73, 8, 9]) and this supports the 
idea that, at least in 2D, enstrophy undergoes a concentrative process with qualities similar to a cascade.

In 3D MHD the situation is further complicated by stretching effects which can contribute to or detract from the concentrative process. Indeed, it is possible for the concentration to occur independently of inertial effects if enstrophy is depleted at large scales and sourced at small scales (this is why the concentrative process is distinct from the notion of "cascade"). Our interest is whether or not, in a circumstance where non-inertial effects experience a hybrid geometric/smoothness based depletion, the enstrophy transport is concentrative.

The results in this section are directed at two conclusions: (1) that the enstrophy flux is predominantly oriented from large to small scale structures across a range of scales and (2) that the enstrophy flux is nearly constant across this range. These indicate that the inter-scale transport of enstrophy is cascade-like (even if the apparent evolution of enstrophy does not display this) and is consistent with the concentrative picture highlighted above provided additional non-linear effects creation, stretching, or dissipation of enstrophy - are depleted by current sheet geometry or positively contribute only at small-scales. This also indicates that the inertial transport of enstrophy is active in that fluid advection effects the structure of current sheets.

These conclusions will be established using the same dynamic, multiscale averaging process described in Section 2.2.1 of this chapter. Here this device acts as a detector of significant sign-fluctuations associated with the enstrophy flux density at a given scale to show that the enstrophy flux is, in a statistically significant sense, oriented from larger to smaller scales, thereby indicating a concentration effect toward structures of progressively fine scale is transpiring. To achieve this we will establish several dynamic estimates for quantities associated with noninertial terms (originating in the current-vorticity formulation of 3D MHD; see Sections 2.3.2 and 2.3.3) and we include several assumptions (see (A1)-(A3) in Section 2.3.1) to accommodate these estimates. Chief among these is a requirement, (A1), that the vorticity field satisfies a hybrid geometric/smoothness property in the region of high spatial complexity. Requirements of this type (i.e. conditions depleting nonlinearities) have been used to formulate conditional regularity results for both 3D NSE (cf. [34, 5]) and 3D MHD (cf. [108, 107, 65]) and our particular formulation is chosen for its robustness with regard to 
its mathematical applications. We include a comment highlighting a more complicated but potentially more physically motivated configuration with the same mathematical effects (see Remark 2.3.1 following our statement of assumption (A1)).

Scale-locality of the enstrophy flux is included as a direct corollary of our main result (see Section 2.3.4). Even for cascading quantities, the locality question is generally more complicated in 3D MHD than in 3D NSE due to the variety of transporting mechanisms (cf. [2, 3]). Under the same conditions that indicate the inertial transfer of enstrophy is concentrative, we affirm that the inertially driven inter-scale transfer is predominantly between comparable scales and, moreover, this locality propagates exponentially along the dyadic scale.

\subsubsection{Enstrophy concentration}

Our mathematical setting is again that of weak solutions to the magnetohydrodynamic equations. Taking the curl of MHD we obtain an evolution equation for the vorticity, $\omega=\nabla \times u$,

$$
\partial_{t} \omega-\Delta \omega=-(u \cdot \nabla) \omega+(\omega \cdot \nabla) u+(b \cdot \nabla) j-(j \cdot \nabla) b
$$

as well as for the current, $j=\nabla \times b$,

$$
\begin{aligned}
\partial_{t} j-\Delta j= & -(u \cdot \nabla) j+(j \cdot \nabla) u+(b \cdot \nabla) \omega-(\omega \cdot \nabla) b \\
& +2 \sum_{l=1}^{3} \nabla b_{l} \times \nabla u_{l} .
\end{aligned}
$$

In our study we substitute for the inward kinetic and magnetic enstrophy fluxes through the boundary of a ball, $B=B\left(x_{0}, 2 R\right)$,

$$
\begin{aligned}
-\int_{\partial B} \frac{1}{2}|\omega|^{2}(u \cdot n) d \sigma & =-\int_{B}(u \cdot \nabla) \omega \cdot \omega d x, \\
-\int_{\partial B} \frac{1}{2}|j|^{2}(u \cdot n) d \sigma & =-\int_{B}(u \cdot \nabla) j \cdot j d x,
\end{aligned}
$$

the inwardly oriented kinetic and magnetic enstrophy fluxes through a shell $S\left(x_{0}, R, 2 R\right)$ by incorporating a (nearly radial) refined cut-off function, $\phi$ exhibiting the properties specified in Section 2.2.1. After multiplying $(u \cdot \nabla) \omega$ and $(u \cdot \nabla) j$ respectively by $\phi \omega$ and $\phi j$, we have 
the following realization of the local kinetic and magnetic enstrophy fluxes at scale $R$ around the point $x_{0}$,

$$
\begin{aligned}
\Phi_{x_{0}, R}^{\omega} & :=\int \frac{1}{2}|\omega|^{2}(u \cdot \nabla \phi) d x=-\int(u \cdot \nabla) \omega \cdot(\phi \omega) d x, \\
\Phi_{x_{0}, R}^{j} & :=\int \frac{1}{2}|j|^{2}(u \cdot \nabla \phi) d x=-\int(u \cdot \nabla) j \cdot(\phi j) d x,
\end{aligned}
$$

and we define the local combined enstrophy flux by $\Phi_{x_{0}, R}=\Phi_{x_{0}, R}^{\omega}+$ $\Phi_{x_{0}, R}^{j}$. Formulas for the localized enstrophy fluxes are realized via the non-linear terms $(u \cdot \nabla) \omega$ and $(u \cdot \nabla) j$ by multiplying $(2.3 .1)$ and (2.3.2) respectively by $\phi \omega$ and $\phi j$ and integrating. In this manner we see that the localized kinetic enstrophy flux is given by,

$$
\begin{aligned}
F^{\omega}(t):= & \int_{0}^{t} \int \frac{1}{2}|\omega|^{2}(u \cdot \nabla \phi) d x d s \\
= & \int \frac{1}{2}|\omega(x, t)|^{2} \psi(x) d x+\int_{0}^{t} \int|\nabla \omega|^{2} \phi d x d s \\
& -\int_{0}^{t} \int \frac{1}{2}|\omega|^{2}\left(\partial_{s} \phi+\Delta \phi\right) d x d s-\int_{0}^{t} \int(\omega \cdot \nabla) u \cdot(\phi \omega) d x d s \\
& -\int_{0}^{t} \int(b \cdot \nabla) j \cdot(\phi \omega) d x d s+\int_{0}^{t} \int(j \cdot \nabla) b \cdot(\phi \omega) d x d s \\
= & \int \frac{1}{2}|\omega(x, t)|^{2} \psi(x) d x+\int_{0}^{t} \int|\nabla \omega|^{2} \phi d x d s \\
& +H^{\omega}+N_{1}^{\omega}+L^{\omega}+N_{2}^{\omega},
\end{aligned}
$$


while the localized magnetic enstrophy flux is given by,

$$
\begin{aligned}
F^{j}(t):= & \int_{0}^{t} \int \frac{1}{2}|j|^{2}(u \cdot \nabla \phi) d x d s \\
= & \int \frac{1}{2}|j(x, t)|^{2} \psi(x) d x+\int_{0}^{t} \int|\nabla j|^{2} \phi d x d s \\
& -\int_{0}^{t} \int \frac{1}{2}|j|^{2}\left(\partial_{s} \phi+\Delta \phi\right) d x d s+\int_{0}^{t} \int(\omega \cdot \nabla) b \cdot(\phi j) d x d s \\
& -\int_{0}^{t} \int(b \cdot \nabla) \omega \cdot(\phi j) d x d s-\int_{0}^{t} \int(j \cdot \nabla) u \cdot(\phi j) d x d s \\
& -\int_{0}^{t} \int\left(2 \sum_{l=1}^{3} \nabla u_{l} \times \nabla b_{l}\right) \cdot(\phi j) d x d s \\
= & \int \frac{1}{2}|j(x, t)|^{2} \psi(x) d x+\int_{0}^{t} \int|\nabla j|^{2} \phi d x d s \\
& +H^{j}+N_{1}^{j}+L^{j}+N_{2}^{j}+X,
\end{aligned}
$$

and we label their combination as $F(t)=F^{\omega}(t)+F^{j}(t)$.

To establish the concentrative effect of inertial forces on the combined enstrophy we will show that the ensemble averages of localized spatio-temporal averages of the above densities associated with an arbitrary $\left(K_{1}, K_{2}\right)$-cover are positive and nearly constant across a range of scales. Before formulating our assumptions we specify several technical values. Fix a value $K_{*}$ so that,

$$
K_{*} \geq \max \left\{\left(K_{1} K_{2}\right)^{1 / 2}, 3 K_{2} / 4, K_{1}\right\}
$$

and set,

$$
\alpha=4 K_{P} K_{*}^{2},
$$

where $K_{P}$ is a constant which will be quantified in Section 2.3.3.

Our assumptions are:

(A1) Hybrid Geometric/Smoothness Assumption. It is assumed for some threshold $M>0$ that we have,

$$
|\omega(x+y, t)-\omega(x, t)| \leq|\omega(x+y, t)||y|^{\frac{1}{2}},
$$

provided $|y|<2 R_{0}+R_{0}^{\frac{2}{3}}, x$ in $\{|\nabla u|>M\}$, and $\omega(x+y) \neq 0$. 
Remark 2.3.1. This assumption is less satisfying than its analogue in the fluid case of [45] where it was sufficient to assume the numerically and observationally motivated assumption of coherence of the direction of vorticity as this depleted the only noninertial effect (that of vortex stretching). In our setting, coherence of the vorticity does not deplete all non-inertial effects but (A1) does.

It is worth mentioning another sufficient formulation as it may be more physically appropriate if the current field is less volatile than the vorticity field. In the modified formulation we assume the vorticity field satisfies a directional coherence condition identical to that in [45] and the current satisfies a hybrid geometric/smoothness condition (like (A1) but with $\omega$ replaced with $j$ ). The assumption on $j$ depletes all non-linear terms except the vortex stretching term via a kinematic argument which mirrors that given in the next section for $\omega$ while the vortex stretching term is depleted by the coherency assumption. This alternative configuration is motivated by the 2D dynamics (cf. [8]) where the current and vorticity concentrate on largely overlapping regions. Here, the current assumes the structure of an elongated monopole while the vorticity-structure consists of four monopoles squished together in a grid so that the orientation of the vorticity is opposite on adjacent monopoles. Extending this intuition to $3 \mathrm{D}$ hints that the current is less oscillatory than the vorticity.

(A2) Modified Kraichnan-Type Scale. Let $e_{0}, E_{0}$, and $P_{0}$ denote the time averaged total energy, total enstrophy, and modified total palenstrophy at the integral scale. Precisely,

$$
\begin{aligned}
e_{0} & =\frac{1}{T} \int_{0}^{T} \frac{1}{R_{0}^{3}} \int \phi_{0}^{4 \rho-3}\left(\frac{|u|^{2}}{2}+\frac{|b|^{2}}{2}\right) d x d s \\
E_{0} & =\frac{1}{T} \int_{0}^{T} \frac{1}{R_{0}^{3}} \int \phi_{0}^{2 \rho-1}\left(|\omega|^{2}+|j|^{2}\right) d x d s
\end{aligned}
$$


and,

$$
\begin{aligned}
P_{0}= & \frac{1}{T} \int_{0}^{T} \frac{1}{R_{0}^{3}} \int \phi_{0}\left(|\nabla \omega|^{2}+|\nabla j|^{2}\right) d x d s \\
& +\frac{1}{T R_{0}^{3}} \int \frac{1}{2}|\omega(x, T)|^{2} \psi_{0}(x) d x .
\end{aligned}
$$

The modification of palenstrophy is due to the nature of the temporal cut-off; in addition, note that the cut-off's are modified for technical reasons and $\rho$ was specified in the construction of these functions.

Set,

$$
\mathcal{E}_{0}=\left(\frac{E_{0}}{P_{0}}\right)^{\frac{1}{2}}
$$

and,

$$
\varepsilon_{0}=\left(\frac{e_{0}}{P_{0}}\right)^{\frac{1}{4}} .
$$

Define the modified Kraichnan-type scale $\sigma_{0}$ by,

$$
\sigma_{0}=\max \left\{\mathcal{E}_{0}, \varepsilon_{0}\right\}
$$

Our assumption (A2) is that, for $\beta=\beta\left(M, K_{1}, K_{2}, \int_{0}^{T}\|\omega\|_{2}^{2} d t\right)$ with $0<\beta<1$ (the precise value will be identified later), we have,

$$
\sigma_{0}<\beta R_{0}
$$

Remark 2.3.2. The Kraichnan-type scale determines the lower limit of scales at which the concentrative effect is affirmed. For us this is realized by restricting to scales $R$ with $\sigma_{0} / \beta<R$. In comparison to the analogous and identically named parameter in the 3D NSE case we here see a correction of $\beta$ by a power of $1 / 2$ necessitated by the emergence of energy-level quantities in Section 2.3.2.

(A3) Localization and Modulation - Because $\int_{0}^{T}\|\omega\|_{2}^{2} d s$ is an $a$ priori bounded quantity (cf. [96]), for a given constant $C_{0}>0$ there exists $R_{0}^{*}$ so that, for any $R_{0} \leq R_{0}^{*}$, we have,

$$
\left(\int_{0}^{T}\|\omega\|_{L^{2}\left(B\left(0,2 R_{0}+R_{0}^{\frac{2}{3}}\right)\right)}^{2} d s\right)^{1 / 2} \leq \frac{1}{C_{0}} .
$$


The localization assumption on $R_{0}$, the radius of the integral scale, is that, for $C_{0}=\alpha$, we have $R_{0} \leq R_{0}^{*}$. A precise (up to certain parameters) value for $\alpha$ will materialize in the proof.

The modulation assumption imposes a restriction on the time evolution of the integral-scale kinetic and magnetic enstrophies across $(0, T)$ consistent with our choice of the temporal cut-off. Precisely,

$$
\begin{gathered}
\int|\omega(x, T)|^{2} \psi_{0}(x) d x \geq \frac{1}{2} \sup _{s} \int|\omega(x, s)|^{2} \psi_{0}(x) d x, \\
\int|j(x, T)|^{2} \psi_{0}(x) d x \geq \frac{1}{2} \sup _{s} \int|j(x, s)|^{2} \psi_{0}(x) d x .
\end{gathered}
$$

Remark 2.3.3. Regarding localization, we have essentially introduced an upper bound on the range of scales across which the concentrative effect is affirmed and note that this restriction is largely technical (see [45] for a preliminary statement of a lemma which will show that the near-constancy across a bounded range of scales extends to a range above that bound).

Using the terminologies of the above assumptions we are ready to state our main result which establishes the positivity and nearconstancy of the combined enstrophy flux across a range of physical scales, the implication of which is that the combined enstrophy is concentrated by inertial forces.

Theorem 2.3.1. If a weak solution $u, b$ of $3 D$ MHD satisfies (A1)-(A3) on $B\left(0,2 R_{0}\right) \times(0, T)$ then,

$$
\frac{1}{4 K_{*}} P_{0} \leq\langle\Phi\rangle_{R} \leq 4 K_{*} P_{0}
$$

for all $\frac{\sigma_{0}}{\beta} \leq R \leq R_{0}$ and $K_{*}$ dependent only on $K_{1}$ and $K_{2}$.

Remark 2.3.4. It will be plain that the localization estimates to be presented in the following section imply that (A1) alone guarantees smoothness over the spatio-temporal integral domain; hence, we are effectively concerned with the global-in-space $\left(\mathbb{R}^{3}\right)$ weak solutions that are smooth over the integral domain. However, since we do not impose any boundary conditions on the integral domain, the control over the 'smooth' norms is strictly local. 
Before continuing to the proof of Theorem 2.3.1 we observe that enstrophy flux locality - i.e. that the transport of the combined enstrophy is predominantly between scales of comparable size - is an immediate corollary. Discussion of this corollary and its precise statement is withheld until Section 6.

To prove Theorem 2.3.1, we will confine ensemble averages of the localized (to a ball of radius $R$ centered at $x_{0}$ ) combined enstrophy flux between scale- and cover-independent multiples of the localized (to the integral domain) total-palenstrophy, $P_{0}$. Local estimates to this effect are contained in Section 2.3.2. Based on these, in Section 2.3.3, the ensemble averaging methodology is applied to complete the proof of Theorem 2.3.1.

\subsubsection{Estimates}

In this section each of the terms from (2.3.3) and (2.3.4) are bounded by quantities which can be related to $e_{0}, E_{0}$, and $P_{0}$ via the apparatus of ensemble averaging with respect to $\left(K_{1}, K_{2}\right)$-covers at scale $R$. Throughout, we limit our consideration to a fixed ball of radius $R$ and suppress the corresponding subscripts. We label various constants by $K, K_{e}, K_{E}$, and $K_{P}$ and note these are dependent on $K_{1}, K_{2}$ and quantities determined by structural properties of 3D MHD.

Bounds for the linear terms in (2.3.3) and (2.3.4) follow simply from properties of the spatial cut-off (see (2.4.3)), i.e.,

$$
H:=H^{\omega}+H^{j} \leq \frac{K_{E}}{R^{2}} \int_{0}^{T} \int \phi^{2 \rho-1}\left(|\omega|^{2}+|j|^{2}\right) d x d s .
$$

Before proceeding to bound the nonlinear terms a digression is necessary to introduce the kinematic framework derived in [32] and adapted to MHD in [107] and [65]. Recall that the deformation tensor of the velocity field can be decomposed in terms of a symmetric component, the strain tensor of $u, S$, and a skew component, $\omega \times \cdot$. Put precisely,

$$
\nabla u=S-\frac{1}{2} \omega \times
$$

The operators in the above decomposition have the following singular 
integral representations:

$$
\omega(x)=\frac{1}{4 \pi} P . V . \int \sigma(\hat{y}) \omega(x+y) \frac{d y}{|y|^{3}},
$$

and,

$$
S(x)=\frac{3}{4 \pi} P . V . \quad \int M(\hat{y}, \omega(x+y)) \frac{d y}{|y|^{3}},
$$

for,

$$
\hat{y}=\frac{y}{|y|}, \sigma(\hat{y})=3 \hat{y} \otimes \hat{y}-I,
$$

and,

$$
M(\hat{y}, f)=\frac{1}{2}(\hat{y} \otimes(\hat{y} \times f)+(\hat{y} \times f) \otimes \hat{y}) .
$$

A key feature for our treatment of the term $\nabla u_{l}$ will follow from the fact that $\sigma$ and $M$ (the latter when $f$ is held constant as a function of $y$ ) have mean zero on the unit sphere. Integral operators such as these are discussed in [103] and [101]. We connect the above to the term $\nabla u_{l} \times \nabla b_{l}$ by noting for the unit vector $e_{l}$ we have,

$$
\nabla u_{l}=S u e_{l}-\frac{1}{2} \omega \times e_{l} .
$$

Using the zero mean value property we write,

$$
\begin{aligned}
\left|\nabla u_{l}\right| \leq & \mid P . V . \int_{|y|<R^{2 / 3}}\left(\frac{1}{4 \pi} \sigma(\hat{y})(\omega(x+y)-\omega(x))\right. \\
& \left.+\frac{3}{4 \pi} M(\hat{y}, \omega(x+y)-\omega(x))\right) \frac{d y}{|y|^{3}} \mid \\
& +\left|\int_{|y| \geq R^{2 / 3}}\left(\frac{1}{4 \pi} \sigma(\hat{y}) \omega(x+y)+\frac{3}{4 \pi} M(\hat{y}, \omega(x+y))\right) \frac{d y}{|y|^{3}}\right| \\
= & I_{1}+I_{2} .
\end{aligned}
$$

Our treatment is now divided between $I_{1}$ and $I_{2}$. For the former, the hybrid geometric-smoothness assumption (A1) entails that,

$$
\begin{aligned}
I_{1} & \leq K(\sigma, M) \int_{|y| \leq R^{2 / 3}}|\omega(x+y)-\omega(x)| \frac{d y}{|y|^{3}} \\
& \leq K(\sigma, M) \int_{|y| \leq R^{2 / 3}}|\omega(x+y)| \frac{d y}{|y|^{5 / 2}},
\end{aligned}
$$


and, therefore, by the Hardy-Littlewood-Sobolev inequality (cf. Chapter $\mathrm{V}$ of [103]),

$$
\left\|I_{1}\right\|_{3} \leq K\|\omega\|_{L^{2}\left(B\left(x_{i}, R^{2 / 3}\right)\right)} .
$$

Regarding $I_{2}$, Hölder's inequality allows that,

$$
\begin{aligned}
I_{2} & \leq K(\sigma, M) \int_{|y| \geq R^{2 / 3}} \frac{1}{|y|^{2}} \frac{\omega(x+y)}{|y|} d y \\
& \leq K(\sigma, M)\left(\int_{|y| \geq R^{2 / 3}} \frac{1}{|y|^{4}} d y\right)^{\frac{1}{2}}\left(\int_{|y| \geq R^{2 / 3}} \frac{|\omega(x+y)|^{2}}{|y|^{2}} d y\right)^{\frac{1}{2}} \\
& \leq K(\sigma, M) \frac{1}{R^{1 / 3+2 / 3}}\|\omega\|_{L^{2}\left(\mathbb{R}^{3}\right)}=\frac{K}{R}\|\omega\|_{L^{2}\left(\mathbb{R}^{3}\right)}
\end{aligned}
$$

Note that we can apply the exact same argument to $\omega$ and obtain,

$$
\begin{aligned}
\omega(x)= & \frac{1}{4 \pi} P . V \cdot \int_{|y|<R^{2 / 3}} \sigma(\hat{y})(\omega(x+y)-\omega(x)) \frac{d y}{|y|^{3}} \\
& +\frac{1}{4 \pi} \int_{|y| \geq R^{2 / 3}} \sigma(\hat{y}) \omega(x+y) \frac{d y}{|y|^{3}} \\
\leq & K\|\omega\|_{L^{2}\left(B\left(x_{i}, R^{2 / 3}\right)\right.}+\frac{K}{R}\|\omega\|_{L^{2}\left(\mathbb{R}^{3}\right)}
\end{aligned}
$$

We are now ready to establish bounds on the coupled non-linear terms and start with the most involved, the term involving $\nabla u_{l} \times \nabla b_{l}$, that labelled $X$, as this will illustrate many of the computational steps necessary for the other terms. Here we show that,

$$
\begin{aligned}
& \int_{0}^{T} \int \phi j \cdot \nabla u_{l} \times \nabla b_{l} d x d s \\
& \leq \frac{K_{P}}{\alpha}\left(\frac{1}{2} \sup _{s}\left\|\psi^{\frac{1}{2}} j\right\|_{2}^{2}+\int_{0}^{T} \int \phi|\nabla j|^{2} d x d s\right) \\
& \quad+\frac{K_{E}}{R^{2}} \int_{0}^{T} \int \phi^{2 \rho-1}|j|^{2} d x d s \\
& \quad+\frac{\alpha^{2} K_{e}}{R^{4}} \int_{0}^{T} \int \phi^{4 \rho-3} \frac{|b|^{2}}{2} d x d s
\end{aligned}
$$

and begin by splitting the spatial integral across the regions where $|\nabla u| \geq M$ and its complement. Considering the complement, we see 
that,

$$
\begin{aligned}
& \int_{0}^{T} \int_{|\nabla u| \leq M} \phi j \cdot \nabla u_{l} \times \nabla b_{l} d x d s \\
& \leq \int_{0}^{T} \int_{|\nabla u| \leq M} M\left|\phi^{1 / 2} j\right|\left|\phi^{1 / 2} \nabla b_{l}\right| d x d s \\
& \leq K \frac{M^{2}}{R^{2}} \int_{0}^{T} \int \phi^{2 \rho-1}|j|^{2} d x d s+\frac{1}{R^{2}} \int_{0}^{T} \int \phi^{2 \rho-1}\left|\nabla b_{l}\right|^{2} d x d s . \\
& \leq \frac{1}{\alpha} \int_{0}^{T}\left\|\phi^{\frac{1}{2}} \nabla j\right\|_{2}^{2} d s+\frac{M^{2}}{R^{2}} \int_{0}^{T} \int \phi^{2 \rho-1}|j|^{2} d x d s \\
& \quad+\frac{K}{R^{4}} \int_{0}^{T} \int \phi^{4 \rho-3}|b|^{2} d x d s
\end{aligned}
$$

The second integral above can be expressed in terms of energy and palenstrophy level terms. Indeed, for $k, h$, and $l$ distinct elements of $\{1,2,3\}$, we have that,

$$
\begin{aligned}
\int_{0}^{T} \int \phi^{2 \rho-1}\left(\partial_{i} b_{l}\right)^{2} d x d s \\
=-\int_{0}^{T} \int \phi^{2 \rho-1} b_{l} \partial_{i} \partial_{i} b_{l} d x d s-\int_{0}^{T} \int \partial_{i} \phi^{2 \rho-1} b_{l} \partial_{i} b_{l} d x d s \\
=\int_{0}^{T} \int \phi^{2 \rho-1} b_{l}\left(\partial_{k} j_{h}-\partial_{h} j_{k}\right) d x d s+\frac{1}{2} \int_{0}^{T} \int \partial_{i} \partial_{i} \phi^{2 \rho-1} b_{l}^{2} d x d s \\
\leq 2 \int_{0}^{T} \int \phi^{2 \rho-1}|b||\nabla j| d x d s \\
\quad+\frac{1}{2} \int_{0}^{T} \int\left|\partial_{i} \partial_{i} \phi^{2 \rho-1}\right||b|^{2} d x d s \\
\leq \int_{0}^{T} \int^{\left(2 \alpha^{\frac{1}{2}} \phi^{2 \rho-3 / 2}|b|\right)\left(\alpha^{-\frac{1}{2}} \phi^{1 / 2}|\nabla j|\right) d x d s} \\
\quad+\frac{K}{R^{2}} \int_{0}^{T} \int \phi^{4 \rho-3}|b|^{2} d x d s .
\end{aligned}
$$

Applying Young's inequality to the first term of (2.3.14) leads to a final 
bound for the case when $|\nabla u|<M$, namely,

$$
\begin{aligned}
& \int_{0}^{T} \int_{|\nabla u| \leq M} \phi j \cdot \nabla u_{l} \times \nabla b_{l} d x d s \\
& \leq \frac{1}{\alpha} \int_{0}^{T} \int \phi|\nabla j|^{2} d x d s+\frac{K_{E}}{R^{2}} \int_{0}^{T} \int \phi^{2 \rho-1}|j|^{2} d x d s \\
& \quad+\frac{\alpha K_{e}}{R^{4}} \int_{0}^{T} \int \phi^{4 \rho-3}|b|^{2} d x d s . \\
& \leq \frac{1}{\alpha}\left(\frac{1}{2} \sup _{s}|| \psi^{\frac{1}{2}} j \|_{2}^{2}+\int_{0}^{T} \int \phi|\nabla j|^{2} d x d s\right) \\
& \quad+\frac{K_{E}}{R^{2}} \int_{0}^{T} \int \phi^{2 \rho-1}|j|^{2} d x d s+\frac{\alpha^{2} K_{e}}{R^{4}} \int_{0}^{T} \int \phi^{4 \rho-3} \frac{|b|^{2}}{2} d x d s .
\end{aligned}
$$

Looking now at the regions of high spatial complexity - i.e. where $|\nabla u| \geq M-$ we split into two cases using the decomposition for $\nabla u_{l}$,

$$
\begin{aligned}
& \left|\int_{0}^{T} \int_{|\nabla u| \geq M} \phi j \cdot \nabla u_{l} \times \nabla b_{l} d x d s\right| \\
& \leq \int_{0}^{T} \int_{|\nabla u| \geq M} I_{1}\left|\phi^{1 / 2} \nabla b_{l}\right|\left|\phi^{1 / 2} j\right| d x d s \\
& \quad+\int_{0}^{T} \int_{|\nabla u| \geq M} I_{2}\left|\phi^{1 / 2} \nabla b_{l}\right|\left|\phi^{1 / 2} j\right| d x d s .
\end{aligned}
$$

For the integral involving $I_{1}$, the hybrid geometric/smoothness assumption plus the localization and modulation assumptions will serve to minimize palenstrophy level terms. Applications of Hölder's inequality and the bound (2.3.8) and subsequently the Gagliardo-Nirenberg inequality 
and Young's inequality gives an initial bound,

$$
\begin{aligned}
& \int_{0}^{T} \int_{|\nabla u| \geq M} I_{1}\left|\phi^{1 / 2} \nabla b_{l} \| \phi^{1 / 2} j\right| d x d s \\
& \leq K\left(\int_{0}^{T}\|\omega\|_{L^{2}\left(B\left(x_{i}, R^{2 / 3}\right)\right.}^{2} d s\right)^{\frac{1}{2}}\left(\int_{0}^{T}\left\|\phi^{\frac{1}{2}} \nabla b_{l}\right\|_{6}^{2}\left\|\phi^{\frac{1}{2}} j\right\|_{2}^{2} d s\right)^{\frac{1}{2}} \\
& \leq \frac{1}{\alpha}\left(\frac{1}{2} \sup _{s}\left\|\psi^{\frac{1}{2}} j\right\|_{2}^{2}+K \int_{0}^{T}\left\|\nabla\left(\phi^{\frac{1}{2}} \nabla b_{l}\right)\right\|_{2}^{2} d s\right) \\
& \leq \frac{1}{\alpha}\left(\frac{1}{2} \sup _{s}\left\|\psi^{\frac{1}{2}} j\right\|_{2}^{2}+K \int_{0}^{T}\left\|\nabla \phi^{\frac{1}{2}} \otimes \nabla b_{l}\right\|_{2}^{2} d s\right. \\
& \left.\quad+K \int_{0}^{T}\left\|\phi^{\frac{1}{2}} \nabla \nabla b_{l}\right\|_{2}^{2} d s\right)
\end{aligned}
$$

where the constant $\alpha$ emerges from the localization assumption, (A3). We further decompose the last two terms. For the first, by expanding the integral, using the bound (2.3.13), applying Young's inequality, and employing the properties of our cut-off functions, we obtain the estimate,

$$
\begin{aligned}
\frac{K}{\alpha} \int_{0}^{T}\left\|\nabla \phi^{\frac{1}{2}} \otimes \nabla b_{l}\right\|_{2}^{2} d s= & \frac{K}{\alpha} \int_{0}^{T} \int\left(\partial_{i} \phi^{\frac{1}{2}}\right)^{2}\left(\partial_{j} b_{l}\right)^{2} d x d s \\
\leq & \frac{K}{\alpha R^{2}} \int_{0}^{T} \int \phi^{2 \rho-1}\left(\partial_{j} b_{l}\right)^{2} d x d s \\
\leq & \frac{K}{\alpha R^{2}} \int_{0}^{T} \int \phi^{2 \rho-1}|b||\nabla j| d x d s \\
& +\frac{K}{\alpha R^{2}} \int_{0}^{T} \int\left|\partial_{i} \partial_{i} \phi^{2 \rho-1}\right||b|^{2} d x d s \\
\leq & \frac{1}{\alpha} \int_{0}^{T} \int \phi|\nabla j|^{2} d x d s \\
& +\frac{K}{\alpha R^{4}} \int_{0}^{T} \int \phi^{4 \rho-3}|b|^{2} d x d s .
\end{aligned}
$$

The last term of (2.3.16) can be expressed in a similar fashion as the above but with several additional steps. To begin, basic calculus 
leads to the identity,

$$
\begin{aligned}
\left\|\phi^{\frac{1}{2}} \nabla \nabla b_{l}\right\|_{2}^{2}= & \int \phi\left(\partial_{i} \partial_{j} b_{l}\right)^{2} d x \\
= & \int\left(\partial_{i} \partial_{j} \phi\right)\left(\partial_{j} b_{l}\right)\left(\partial_{i} b_{l}\right) d x+\int \phi\left(\partial_{j} \partial_{j} b_{l}\right)\left(\partial_{i} \partial_{i} b_{l}\right) d x \\
& +\int\left(\partial_{j} \phi\right)\left(\partial_{j} b_{l}\right)\left(\partial_{i} \partial_{i} b_{l}\right) d x+\int\left(\partial_{i} \phi\right)\left(\partial_{j} \partial_{j} b_{l}\right)\left(\partial_{i} b_{l}\right) d x
\end{aligned}
$$

the terms of which we now estimate. For the first, using Young's inequality we see that,

$$
\begin{aligned}
\frac{K}{\alpha}\left|\int\left(\partial_{i} \partial_{j} \phi\right)\left(\partial_{j} b_{l}\right)\left(\partial_{i} b_{l}\right) d x\right| & \leq K \int\left|\partial_{i} \partial_{j} \phi\right|\left|\partial_{j} b_{l}\right|\left|\partial_{i} b_{l}\right| d x \\
& \leq \frac{K}{R^{2}} \int \phi^{2 \rho-1}\left|\partial_{j} b_{l}\right|\left|\partial_{i} b_{l}\right| d x \\
& =\frac{K}{R^{2}} \int\left(\phi^{\rho-1 / 2}\left|\partial_{j} b_{l}\right|\right)\left(\phi^{\rho-1 / 2}\left|\partial_{i} b_{l}\right|\right) d x \\
& \leq \frac{K}{R^{2}} \int \phi^{2 \rho-1}\left|\nabla b_{l}\right|^{2} d x
\end{aligned}
$$

Moving on, since $b$ is divergence free and assuming $k, h$, and $l$ are distinct, we have,

$$
\begin{aligned}
& \frac{K}{\alpha}\left|\int\left(\partial_{j} \phi\right)\left(\partial_{j} b_{l}\right)\left(\partial_{i} \partial_{i} b_{l}\right) d x\right| \\
& =\frac{K}{\alpha} \int\left|\partial_{j} \phi\right|\left|\partial_{j} b_{l}\right|\left|\partial_{h} j_{k}-\partial_{k} j_{h}\right| d x \\
& \leq \frac{K}{\alpha} \int\left|\partial_{j} \phi\right|\left|\partial_{j} b_{l}\right|\left|\partial_{h} j_{k}\right| d x \\
& \leq \int\left(\frac{K}{\alpha^{\frac{1}{2}} R} \phi^{\rho-1 / 2}\left|\partial_{j} b_{l}\right|\right)\left(\frac{1}{\alpha^{\frac{1}{2}}} \phi^{\frac{1}{2}}\left|\partial_{h} j_{k}\right|\right) d x \\
& \leq \frac{K}{\alpha R^{2}} \int \phi^{2 \rho-1}\left|\nabla b_{l}\right|^{2} d x+\frac{1}{2 \alpha} \int \phi|\nabla j|^{2} d x \\
& \leq \frac{K}{R^{4}} \int \phi^{4 \rho-3}|b|^{2} d x+\frac{1}{\alpha} \int \phi|\nabla j|^{2} d x .
\end{aligned}
$$

Using Young's inequality and properties of the cut-off function, the 
remaining term is bounded as,

$$
\begin{aligned}
\frac{K}{\alpha}\left|\int \phi\left(\partial_{j} \partial_{j} b_{l}\right)\left(\partial_{i} \partial_{i} b_{l}\right) d x\right| & \leq \frac{K}{\alpha} \int \phi\left(\partial_{i} \partial_{i} b_{l}\right)^{2} d x+\frac{K}{\alpha} \int \phi\left(\partial_{j} \partial_{j} b_{l}\right)^{2} d x \\
& \leq \frac{K_{P}}{\alpha} \int \phi|\nabla j|^{2} d x
\end{aligned}
$$

Combining the above (and observing $\alpha>1$ ) leads to a final bound for the term involving $I_{1}$, namely,

$$
\begin{aligned}
& \int_{0}^{T} \int_{|\nabla u| \geq M} \phi j \cdot \nabla u_{l} \times \nabla b_{l} d x d s \\
& \leq \frac{K_{P}}{\alpha}\left(\frac{1}{2} \sup _{s}|| \psi^{\frac{1}{2}} j \|_{2}^{2}+\int_{0}^{T} \int \phi|\nabla j|^{2} d x d s\right) \\
& \quad+\frac{\alpha K_{e}}{R^{4}} \int_{0}^{T} \int \phi^{4 \rho-3}|b|^{2} d x d s
\end{aligned}
$$

Turning to the non-singular case of our decomposition of $\left|\nabla u_{l}\right|$, we use $\alpha$ to de-emphasize non-localization-apt palenstrophy level quantities. Its emergence is forced upon an application of Young's inequality with a reciprocal cost to an energy level quantity. Using the direct estimate (2.3.9) on $\left|I_{2}\right|$ and then applying Hölder's inequality, Young's inequality, and the same steps used to obtain and proceed from (2.3.14), we see that,

$$
\begin{aligned}
& \int_{0}^{T} \int_{|\nabla u| \geq M} I_{2}\left|\phi^{1 / 2} \nabla b_{l} \| \phi^{1 / 2} j\right| d x d s \\
& \leq \frac{K}{R}\left(\int_{0}^{T}\|\omega\|_{2}^{2} d s\right)^{\frac{1}{2}}\left(\int_{0}^{T}\left\|\phi^{\frac{1}{2}} j\right\|_{2}^{2}\left\|\phi^{\frac{1}{2}} \nabla b_{l}\right\|_{2}^{2} d s\right)^{\frac{1}{2}} \\
& \leq\left(\frac{1}{\sqrt{\alpha}} \sup _{s}\left\|\psi^{\frac{1}{2}} j\right\|_{2}\right)\left(\frac{\alpha K}{R^{2}} \int_{0}^{T}\left\|\phi^{\frac{1}{2}} \nabla b_{l}\right\|_{2}^{2} d s\right)^{\frac{1}{2}} \\
& \leq \frac{1}{\alpha} \frac{1}{2} \sup _{s}\left\|\psi^{\frac{1}{2}} j\right\|_{2}^{2}+\frac{\alpha K}{R^{2}} \int_{0}^{T}\left\|\phi^{\frac{1}{2}} \nabla b_{l}\right\|_{2}^{2} d s \\
& \leq \frac{1}{\alpha}\left(\frac{1}{2} \sup _{s}\left\|\psi^{\frac{1}{2}} j\right\|_{2}^{2}+\int_{0}^{T} \int \phi|\nabla j|^{2} d x d s\right) \\
& \quad+\frac{\alpha K_{e}}{R^{4}} \int_{0}^{T} \int \phi^{4 \rho-3} \frac{|b|^{2}}{2} d x d s .
\end{aligned}
$$


Combining the bounds (2.3.15), (2.3.17), and (2.3.18) establishes the bound (2.3.11) and concludes our discussion of the term involving $\nabla u_{l} \times \nabla b_{l}$.

Bounds for the remaining four critical order nonlinear terms, $N_{1}^{\omega}$, $N_{2}^{\omega}, N_{1}^{j}$, and $N_{2}^{j}$, are now attended. The processes for estimating $N_{1}^{\omega}$ and $N_{2}^{j}$ are identical up to labeling. We only illustrate the latter. Splitting the space integral depending on the size of $|\nabla u|$ we have, when $|\nabla u|<M$, that,

$$
N_{2}^{j}=\int_{0}^{T} \int_{|\nabla u|<M}(j \cdot \nabla) u \cdot \phi j d x d s \leq M \int_{0}^{T}\left\|\phi^{\frac{1}{2}} j\right\|_{2}^{2} d s .
$$

When $|\nabla u| \geq M$, noting by direct comparison that $|j| \leq \sqrt{5}|\nabla b|$, we bound the quantity,

$$
\int_{0}^{T} \int_{|\nabla u| \geq M}|\nabla u|\left|\phi^{\frac{1}{2}} \sqrt{5} \nabla b \| \phi^{\frac{1}{2}} j\right| d x d s,
$$

in an identical fashion to the term originating from the Lorentz force. We thus obtain that,

$$
\begin{aligned}
N_{2}^{j}= & \int_{0}^{T} \int(j \cdot \nabla) u \cdot \phi j d x d s \\
\leq & \frac{K_{P}}{\alpha}\left(\frac{1}{2} \sup _{s}|| \psi^{\frac{1}{2}} j \|_{2}^{2}+\int_{0}^{T} \int \phi|\nabla j|^{2} d x d s\right) \\
& +\frac{K_{E}}{R^{2}} \int_{0}^{T} \int \phi^{2 \rho-1}|j|^{2} d x d s+\frac{\alpha^{2} K_{e}}{R^{4}} \int_{0}^{T} \int \phi^{4 \rho-3} \frac{|b|^{2}}{2} d x d s
\end{aligned}
$$

and,

$$
\begin{aligned}
N_{1}^{\omega}= & \int_{0}^{T} \int(\omega \cdot \nabla) u \cdot \phi \omega d x d s \\
\leq & \frac{K_{P}}{\alpha}\left(\frac{1}{2} \sup _{s}\left\|\psi^{\frac{1}{2}} \omega\right\|_{2}^{2}+\int_{0}^{T} \int \phi|\nabla \omega|^{2} d x d s\right) \\
& +\frac{K_{E}}{R^{2}} \int_{0}^{T} \int \phi^{2 \rho-1}|\omega|^{2} d x d s+\frac{\alpha^{2} K_{e}}{R^{4}} \int_{0}^{T} \int \phi^{4 \rho-3} \frac{|u|^{2}}{2} d x d s
\end{aligned}
$$

The terms $N_{1}^{j}$ and $N_{2}^{\omega}$ also enjoy formally identical bounding procedures. The spatial integrals are still split into regions depending on 
$|\nabla u|$. When $|\nabla u|<M$ we have the point-wise estimate $|\omega| \leq 5^{1 / 2} M$ and we obtain a scaled version of prior bounds. When $|\nabla u| \geq M$ we use the kinematic estimate, (2.3.10), on $|\omega|$. The same familiar argument now shows that $N_{1}^{j}$ and $N_{2}^{\omega}$ are both bounded by the right hand side of (2.3.19).

To summarize,

$$
\begin{aligned}
N:=N_{1}^{\omega}+N_{2}^{\omega}+N_{1}^{j}+N_{2}^{j} \leq & \frac{K_{P}}{\alpha}\left(\frac{1}{2} \sup _{s}\left(\left\|\psi^{\frac{1}{2}} \omega\right\|_{2}^{2}+\left\|\psi^{\frac{1}{2}} j\right\|_{2}^{2}\right)\right. \\
& \left.+\int_{0}^{T} \int \phi\left(|\nabla \omega|^{2}+|\nabla j|^{2}\right) d x d s\right) \\
& +\frac{K_{E}}{R^{2}} \int_{0}^{T} \int \phi^{2 \rho-1}\left(|\omega|^{2}+|j|^{2}\right) d x d s \\
& +\frac{\alpha^{2} K_{e}}{R^{4}} \int_{0}^{T} \int \phi^{4 \rho-3} \frac{|u|^{2}+|b|^{2}}{2} d x d s .
\end{aligned}
$$

Examining $L^{\omega}$ and $L^{j}$ we note that upon integrating by parts and summing a cancellation occurs leaving us with,

$$
L:=L^{\omega}+L^{j}=\int_{0}^{T} \int(j \cdot \omega)(\nabla \phi \cdot b) d x d s
$$

Although the above term is lower order and can be bounded in a more efficient way than what transpires below, we choose the less direct approach to limit problem specific dependencies of the parameter $\beta$. We again split the spatial integral to obtain on one hand that,

$$
\begin{aligned}
& \int_{0}^{T} \int_{|\nabla u| \leq M}(j \cdot \omega)(\nabla \phi \cdot b) d x d s \\
& \leq \sqrt{5} M \frac{1}{R} \int_{0}^{T}\left\|\phi^{\frac{1}{2}} b\right\|_{2}\left\|\phi^{\rho-1 / 2} j\right\|_{2} d s \\
& \leq \frac{K_{E}}{R^{2}} \int_{0}^{T} \int \phi^{2 \rho-1}|j|^{2} d x d s+\frac{\alpha^{2} K_{e}}{R^{4}} \int_{0}^{T} \int \phi^{4 \rho-3}|b|^{2} d x d s
\end{aligned}
$$

and, on the other hand, using the kinematic decomposition of $\omega$ as a 
singular integral, that,

$$
\begin{aligned}
& \int_{0}^{T} \int_{|\nabla u| \geq M} \int_{|y| \geq R^{2 / 3}} \sigma(\hat{y}) \omega(x+y) \frac{d y}{|y|^{3}}|j||\nabla \phi \cdot b| d x d s \\
& \leq \frac{1}{\alpha} \frac{1}{2} \sup _{s}\left\|\psi^{\frac{1}{2}} j\right\|_{2}^{2} \\
& \quad+\frac{\alpha^{2} K_{e}}{R^{4}} \int_{0}^{T} \int \phi^{4 \rho-3}|b|^{2} d x d s
\end{aligned}
$$

and,

$$
\begin{aligned}
& \int_{0}^{T} \int_{|\nabla u| \geq M} \int_{|y|<R^{2 / 3}} \sigma(\hat{y}) \omega(x+y) \frac{d y}{|y|^{3}}|j||\nabla \phi \cdot b| d x d s \\
& \leq \frac{1}{\alpha}\left(\int_{0}^{T}\left(\left\|\phi^{\frac{1}{2}} j\right\|_{2}\left\||\nabla \phi|^{\frac{1}{2}} b\right\|_{2}\right)^{2} d x d s\right)^{\frac{1}{2}} \\
& \leq \frac{1}{\alpha}\left(\frac{1}{2} \sup _{s}\left\|\psi^{\frac{1}{2}} j\right\|_{2}^{2}+\int_{0}^{T}\left\|\phi^{\frac{1}{2}} \nabla j\right\|_{2}^{2} d x d s\right) \\
& +\frac{\alpha^{2} K_{e}}{R^{4}} \int_{0}^{T} \int \phi^{4 \rho-3}|b|^{2} d x d s
\end{aligned}
$$

Combining the above we conclude that $L$ is also bounded by the quantity given in (2.3.19).

\subsubsection{Proof of Theorem 2.3.1}

This section contains the proof of Theorem 2.3.1. We work in the context of an arbitrary $\left(K_{1}, K_{2}\right)$-cover at scale $R,\left\{B\left(x_{i}, R\right)\right\}_{i=1}^{n}$, of the integral domain $B\left(0, R_{0}\right)$ where $R<R_{0}<1$ and assume the premises of Theorem 2.3.1 hold.

First we establish bounds for averages associated to an arbitrary cover element centered at $x_{i}$ (note that $x_{0}$ was arbitrary in the previous subsection and the bounds were independent of $x_{0}$; our subscripts here indicate localization around $x_{i}$ at scale $R$ ), i.e. for,

$$
\begin{aligned}
\int_{0}^{T} \Phi_{x_{i}, R} d s= & \int \frac{1}{2}|\omega(x, T)|^{2} \psi_{i}(x) d x+\int_{0}^{T} \int|\nabla \omega|^{2} \phi_{i} d x d s \\
& +\int \frac{1}{2}|j(x, T)|^{2} \psi_{i}(x) d x+\int_{0}^{T} \int|\nabla j|^{2} \phi_{i} d x d s \\
& +H_{i}+N_{i}+L_{i}+X_{i} .
\end{aligned}
$$


Observe that,

$$
\begin{aligned}
H_{i} & +N_{i}+L_{i}+X_{i} \\
\leq & \frac{K_{P}}{\alpha}\left(\frac{1}{2}\left(\sup _{s}\left\|\psi_{i}^{\frac{1}{2}} j\right\|_{L^{2}\left(B\left(x_{i}, 2 R\right)\right)}+\sup _{s}\left\|\psi_{i}^{\frac{1}{2}} \omega\right\|_{L^{2}\left(B\left(x_{i}, 2 R\right)\right)}\right)\right. \\
& \left.+\int_{0}^{T} \int \phi_{i}\left(|\nabla j|^{2}+|\nabla \omega|^{2}\right) d x d s\right) \\
& +\frac{K_{E}}{R^{2}} \int_{0}^{T} \int \phi_{i}^{2 \rho-1}\left(|\omega|^{2}+|j|^{2}\right) d x d s \\
& +\frac{\alpha^{2} K_{e}}{R^{4}} \int_{0}^{T} \int \phi_{i}^{4 \rho-3}\left(\frac{|b|^{2}}{2}+\frac{|u|^{2}}{2}\right) d x d s .
\end{aligned}
$$

The properties of $\left(K_{1}, K_{2}\right)$-covers (see (2.2.3)) allow us, upon taking ensemble averages and applying the modulation part of (A3), to pass to a lower bound involving only integral scale quantities,

$$
\left\langle\frac{1}{T} \int_{0}^{T} \frac{1}{R^{3}} \Phi_{x_{i}, R} d s\right\rangle_{R} \geq \frac{1}{K_{1}} P_{0}-K_{2} \frac{K_{P}}{\alpha} P_{0}-\frac{K_{2} K_{E}}{R^{2}} E_{0}-\frac{\alpha^{2} K_{2} K_{e}}{R^{4}} e_{0}
$$

At this point we specify the value for $\alpha$,

$$
\alpha=4 K_{P} K_{*}^{2},
$$

and recall that,

$$
K_{*} \geq \max \left\{\left(K_{1} K_{2}\right)^{1 / 2}, 3 K_{2} / 4, K_{1}\right\}
$$

Consequently,

$$
K_{2} \frac{K_{P}}{\alpha} \leq \frac{1}{4 K_{1}}
$$

and, therefore,

$$
\left\langle\frac{1}{T} \int_{0}^{T} \frac{1}{R^{3}} \Phi_{x_{i}, R} d s\right\rangle_{R} \geq \frac{3}{4 K_{1}} P_{0}-\frac{K_{2} K_{E}}{R^{2}} E_{0}-\frac{\alpha^{2} K_{2} K_{e}}{R^{4}} e_{0} .
$$

A consequence of (A2) is that,

$$
\frac{\alpha^{2} K_{2} K_{e}}{R^{4}} e_{0} \leq \beta^{4} \alpha^{2} K_{2} K_{e} P_{0},
$$


and,

$$
\frac{K_{2} K_{E}}{R^{2}} E_{0} \leq \beta^{2} K_{2} K_{E} P_{0}
$$

Therefore, noting $0<\beta<1$,

$$
\left\langle\frac{1}{T} \int_{0}^{T} \frac{1}{R^{3}} \Phi_{x_{i}, R} d s\right\rangle_{R} \geq \frac{3}{4 K_{1}} P_{0}-\beta^{2} K_{2}\left(K_{E}+\alpha^{2} K_{e}\right) P_{0} .
$$

The parameter $\beta$ which modifies our Kraichnan-type scale is now chosen to be small enough that,

$$
\left.2 \beta^{2} K_{1} K_{2}\left(K_{E}+\alpha^{2} K_{e}\right)\right)<1
$$

Then,

$$
\left\langle\frac{1}{T} \int_{0}^{T} \frac{1}{R^{3}} \Phi_{x_{i}, R} d s\right\rangle_{R} \geq \frac{1}{4 K_{1}} P_{0} \geq \frac{1}{4 K_{*}} P_{0} .
$$

Establishing the upper bound is, by properties of $\left(K_{1}, K_{2}\right)$-covers, immediate because we chose $K_{*}$ so that $4 K_{*} \geq 3 K_{2}$. Indeed,

$$
\left\langle\frac{1}{T} \int_{0}^{T} \frac{1}{R^{3}} \Phi_{x_{i}, R} d s\right\rangle_{R} \leq K_{2} P_{0}+2 P_{0} \leq 4 K_{*} P_{0} .
$$

Having established that,

$$
\frac{1}{4 K_{*}} P_{0} \leq\langle\Phi\rangle_{R} \leq 4 K_{*} P_{0}
$$

the proof is complete.

\subsubsection{Locality of the enstrophy flux}

As mentioned previously, we can immediately deduce locality of the flux from Theorem 2.3.1. Flux locality, in the context of turbulence phenomenology, refers to the fact that the transfer takes place primarily between comparable scales. This is realized in terms of the flux by requiring that the time averaged flux at scale $R$ is well-correlated only with the time averaged fluxes at comparable scales. While flux locality is phenomenologically accepted in the hydrodynamic case, there 
has been some controversy about the locality in plasma turbulence (cf. $[2,3]$ for a locality result on the energy level, as well as a discussion and references on the topic). The present result establishes that the combined enstrophy flux is local.

Define time averaged local magnetic and kinetic enstrophy fluxes associated to the cover element around the point $x_{i}$ as,

$$
\begin{aligned}
\Psi_{x_{i}, R}^{\omega} & =\frac{1}{T} \int_{0}^{T} \int \frac{1}{2}|\omega|^{2}\left(u \cdot \nabla \phi_{i}\right) d x d s=R^{3} \Phi_{x_{i}, R}^{\omega}, \\
\Psi_{x_{i}, R}^{j} & =\frac{1}{T} \int_{0}^{T} \int \frac{1}{2}|j|^{2}\left(u \cdot \nabla \phi_{i}\right) d x d s=R^{3} \Phi_{x_{i}, R}^{j},
\end{aligned}
$$

and, correspondingly, the combined enstrophy flux as $\Psi_{x_{i}, R}=\Psi_{x_{i}, R}^{\omega}+$ $\Psi_{x_{i}, R}^{j}$.

Further, define the ensemble average over a $\left(K_{1}, K_{2}\right)$-cover of the time-averaged combined flux to be,

$$
\langle\Psi\rangle_{R}=\frac{1}{n} \sum_{i=1}^{n} \Psi_{x_{i}, R}=R^{3}\langle\Phi\rangle_{R}
$$

Using the clear relationships between the spatio-temporal and ensemble averaged terms and the time and ensemble averaged terms, one can use the bounds established in Theorem 2.3.1 to directly verify the following theorem (for which the proof is omitted).

Theorem 2.3.2. Let $u$ and $b$ satisfy the assumptions of Theorem 2.3.1 and let $R$ and $r$ be two scales in the range $\sigma_{0} / \beta \leq r, R \leq R_{0}$. Then,

$$
\frac{1}{16 K_{*}^{2}}\left(\frac{r}{R}\right)^{3} \leq \frac{\langle\Psi\rangle_{r}}{\langle\Psi\rangle_{R}} \leq 16 K_{*}^{2}\left(\frac{r}{R}\right)^{3} .
$$

Remark 2.3.5. Note that along the dyadic scale $-r=2^{k} R$ - the locality propagates exponentially.

\subsection{On the temperature variance cascade in SQG turbulence}

In this section we study the dynamical influence of inertial forces on the temperature variance of solutions to the surface quasi-geostrophic 
(SQG) model and its generalizations. In particular, we are interested in the inertial effect of a strongly rotating, stratified fluid $u$ on a surface temperature (or buoyancy) $\theta$ where the fluid's streamfunction, $\Psi$, is independent of the vertical coordinate. The evolution of $\theta$ is governed by the SQG equations which have the form,

$$
\begin{cases}\left(\partial_{t}+u \cdot \nabla+\kappa \Lambda^{\alpha}\right) \theta=0 & \text { in } \mathbb{R}^{2} \times(0, \infty) \\ u=\left(-\partial_{2} \Psi, \partial_{1} \Psi\right), \nabla \cdot u=0 & \text { in } \mathbb{R}^{2} \times(0, \infty) \\ \theta(\cdot, 0)=\theta_{0} & \text { in } \mathbb{R}^{2},\end{cases}
$$

where $0<\alpha \leq 2$ and $\kappa \geq 0$ (which we subsequently take to be 1 ) are parameters, the streamfunction and $\theta$ are related by $\Lambda \Psi=-\theta$, and $\Lambda^{\alpha}$ is defined as a pseudo-differential operator by,

$$
\left(\Lambda^{\alpha} f\right)^{\wedge}(\xi)=|\xi|^{\alpha} f^{\wedge}(\xi)
$$

for $\xi \in \mathbb{R}^{2}$. We will work with $\theta_{0} \in L^{2}\left(\mathbb{R}^{2}\right)$ taking $\theta$ to be a weak solution to (2.4.1) noting that the global-in-time existence of such solutions is well known for all values of $\alpha$ in $(0,2]$.

The SQG equations were introduced in the context of the quasigeostrophic equations (QG), a geophysically relevant 3D model for the displacement of a rotating, stratified fluid from a solid body rotation, to rectify the fact that the QG description of atmospheric and oceanic processes breaks down in regions near the fluid's boundaries such as the troposphere and ocean surface. Essentially, there are various dynamical regimes in the fluid media which demand more nuanced descriptions than that given by QG. For surface proximate flow, this is achieved by assuming the potential vorticity of a QG fluid is uniform. This assumption allows one to derive the SQG equations from QG. These models are also mathematically interesting as, due to the fact that strong stratification makes the systems behave in a quasi-lower dimensional fashion, they provide a potentially more friendly setting (compared to the 3D Navier-Stokes equations or 3D Euler equations) to study the problem of global regularity and, additionally, they serve as a testing ground for turbulence theories (cf. [33, 37, 67]).

A spectral theory of SQG turbulence was initially studied by Blumen in [11] where he derives Kolmogorov-Kraichnan type scaling laws and associated inertial ranges for the inviscid regime (see also [67]). In SQG there are two ideally conserved quantities, the surface temperature variance and the depth-integrated total energy, the densities for 
these quantities are $|\theta|^{2}$ and $|u|^{2}$, which behave in an analogous fashion to 2D fluid enstrophy and energy in that they exhibit direct and inverse cascades respectively. Recent numerical work in [36] supports the picture of intermittently distributed, thinning temperature assemblages for $\alpha \in[0,2]$. Mathematically, the inverse energy cascade in QG turbulence has been studied from a Littlewood-Paley perspective (cf. [33]). The main goal of this section is to present a rigorous affirmation of the existence of the temperature variance cascade and the scale-locality of the temperature variance flux across an inertial range under conditions which are consistent with qualitative properties of SQG turbulence.

The mathematical methodology used is an adaptation of that developed by Dascaliuc and Grujić to study cascades and flux locality in hydrodynamic turbulence $[40,43]$ and extended to study related properties in plasma turbulence [16]. The adaptation is necessary because fractional diffusion complicates our ability to study dynamical properties of the flow via a localized temperature variance equation (or inequality) as, on $\mathbb{R}^{2}$, no dominant, a priori positive dissipative quantity is immediately apparent. To overcome this, we incorporate an operator which extends scalar functions of $\mathbb{R}^{2}$ to $\mathbb{R}^{2} \times(0, \infty)$. The fractional Laplacian of a function on $\mathbb{R}^{2}$ is then recovered via the trace of the normal derivative of the extension. This approach has been applied to obtain regularity results for the critical case in [22] and certain supercritical scenarios in [39].

\subsubsection{Methodology}

In this section we describe the ensemble averaging process which constitutes the framework on which turbulent cascades will be studied. Our methodology is an adaptation of the statistical apparatus of Dascaliuc and Grujić originally developed to study hydrodynamic turbulence (cf. [40]). At its core is a reformulation of the content of Section 2.2.1 but taken over a $2 \mathrm{D}$ macro-scale domain $B\left(x_{0}, R_{0}\right)$, the open ball of radius $R_{0}$ centered at $x_{0}$. Multi-scale, statistical comparisons will be achieved using 2D $\left(K_{1}, K_{2}\right)$-covers which are presently defined.

Definition 2.4.1. Let $K_{1}, K_{2} \in \mathbb{N}$ and $0<R \leq R_{0}$. The cover of the macro-scale domain $B\left(x_{0}, R_{0}\right)$ by the $n$ (open) balls $\left\{B\left(x_{i}, R\right)\right\}_{i=1}^{n}$ is a 
$\left(K_{1}, K_{2}\right)$-cover at scale $R$ if,

$$
\left(\frac{R_{0}}{R}\right)^{2} \leq n \leq K_{1}\left(\frac{R_{0}}{R}\right)^{2}
$$

and, for any $x \in B\left(x_{0}, R_{0}\right), x$ is contained in at most $K_{2}$ cover elements.

Localization is achieved via refined cut-off functions. For a cover element centered at $x_{i}$ of scale $R$, let $\phi_{i}(x, t)=\eta(t) \psi(x)$ where $\eta \in$ $C_{0}^{\infty}(0, \infty)$ and $\psi \in C_{0}^{\infty}\left(\mathbb{R}^{2}\right)$ take values in $[0,1]$ and satisfy,

$$
\eta=0 \text { on }(0, T / 3) \cup(5 T / 3, \infty), \eta=1 \text { on }[2 T / 3,4 T / 3], \frac{\left|\partial_{t} \eta\right|}{\eta^{2 \delta-1}} \leq \frac{C_{0}}{T},
$$

and,

$$
\psi=1 \text { on } B\left(x_{i}, R\right), \frac{\left|\partial_{i} \psi\right|}{\psi^{\delta}} \leq \frac{C_{0}}{R}, \frac{\left|\partial_{i} \partial_{j} \psi\right|}{\psi^{2 \delta-1}} \leq \frac{C_{0}}{R^{2}},
$$

where $1 / 2<\delta<1$ is fixed. We require also that $2 T \geq R_{0}^{\alpha}$ where $\alpha$ reflects the fractional exponent in (2.4.1).

The macro-scale cut-off function, $\phi_{0}$, is a fixed, refined cut-off function for the ball $B\left(x_{0}, R_{0}\right)$ which is supported on $B\left(x_{0}, 2 R_{0}\right)$ having spatial and temporal components $\psi_{0}$ and $\eta_{0}$.

Comparisons between sub-macro-scale quantities and macro-scale quantities will be accommodated by imposing several additional conditions on $\phi_{i}$ when $x_{i}$ lies near the boundary of $B\left(x_{0}, R_{0}\right)$. If $B\left(x_{i}, R\right) \subset$ $B\left(x_{0}, R_{0}\right)$ we assume $\psi \leq \psi_{0}$. Alternatively, when $B\left(x_{i}, R\right) \not \subset B\left(x_{0}, R_{0}\right)$, let $l(x, y)$ denote the collection of points on the line through $x$ and $y$ and define the sets,

$$
\begin{aligned}
S_{0}= & B\left(x_{i}, R\right) \cap B\left(x_{0}, R_{0}\right), \\
S_{1}= & \left\{x: R_{0} \leq|x|<2 R_{0} \text { and } \emptyset \neq\left(l\left(x, x_{0}\right) \cap \partial B\left(x_{i}, R\right) \cap B\left(x_{0}, R_{0}\right)^{c}\right)\right\}, \\
S_{2}= & \left(\left\{x: R_{0} \leq|x|<2 R_{0}, \emptyset \neq\left(l\left(x, x_{0}\right) \cap \partial B\left(x_{i}, 2 R\right) \cap B\left(x_{0}, R_{0}\right)^{c}\right)\right\}\right. \\
& \left.\cup B\left(x_{i}, 2 R\right)\right) \cap\left(S_{0} \cup S_{1}\right)^{c} .
\end{aligned}
$$


Then, assume that $\psi$ satisfies (2.4.3), $\psi=1$ on $S_{0}, \psi=\psi_{0}$ on $S_{1}$, and $\operatorname{supp} \psi=S_{2}$. The above conditions ensure that $\phi \leq \phi_{0}$ and that $\psi$ can be constructed to have an inwardly oriented gradient field.

Extensions into a third spatial dimension will be necessary when deriving a localized temperature variance equality. To accommodate this, fix a vertical scale $R^{*}$ and let $\psi^{*} \in C^{\infty}([0, \infty))$ take the value 1 on $\left[0, R^{*}\right]$ and decrease to 0 across $\left[R^{*}, R^{*}+R_{0}\right)$, in a fashion mirroring the (spatial) gradient restrictions imposed on $\phi_{0}$. Then, the vertical extensions of our refined cut-off functions are just $\phi_{0}^{*}(x, z, t)=\phi_{0}(x, t) \psi^{*}(z)$ and $\phi_{i}^{*}(x, z, t)=\phi_{i}(x, t) \psi^{*}(z)$. Note that the property $\phi_{i}^{*} \leq \phi_{0}^{*}$ is trivially preserved.

The statistical element of the methodology is carefully described in [40] and Section 2.2.1, although some additional remarks are necessary for our present work. Let $g$ be a physical density and denote its localized surface-time average over a cover element at scale $R$ around $x_{i}$ by,

$$
G_{i}=\frac{1}{T} \int_{0}^{2 T} \frac{1}{R^{2}} \int_{B\left(x_{i}, 2 R\right)} g(x, t) \phi_{i}(x, t) d x,
$$

and let $\langle G\rangle_{R}$ denote the arithmetic mean - our version of ensemble averages - over localized averages associated with cover elements,

$$
\langle G\rangle_{R}=\frac{1}{n} \sum_{i=1}^{n} G_{i} .
$$

Assume that $g^{*}$ is an extension of $g$ in the $z$-direction. Analogous quantities involving the vertical extensions of cover elements are,

$$
G_{i}^{*}=\frac{1}{T} \int_{0}^{2 T} \frac{1}{R^{2}} \int_{B\left(x_{i}, 2 R\right)}\left[\int_{0}^{R^{*}+R_{0}} g^{*}(x, z, t) \phi_{i}^{*}(x, z, t) d z\right] d x d t .
$$

Note that no normalization is carried out in the $z$-direction. This is consistent with the fact that the extended cut-off functions all have the same vertical scale.

We extract statistical information about $g$ at the scale $R$ by studying the collection of all ensemble averages at the given scale. In particular, for an a priori sign varying density, if all covers taken at scale $R$ admit ensemble averages which are mutually comparable and positive, e.g.,

$$
\frac{1}{C} K \leq\langle G\rangle_{R} \leq C K,
$$


for positive, cover independent constants $C$ and $K$, then this density is essentially positive (i.e. positive in a statistical sense) at scale $R$.

\subsubsection{The localized temperature variance flux}

Recall that the temperature variance flux through the boundary of a region $B$ is,

$$
\int_{\partial B} \frac{1}{2} \theta^{2} u \cdot \hat{n} d s=\int_{B}(u \cdot \nabla \theta) \theta d x
$$

where $\hat{n}$ is the outward normal vector. The refined cut-off functions were constructed so that the gradient field $\nabla \phi_{i}$ was oriented roughly toward the center of the ball $B\left(x_{i}, R\right)$. This leads to the following realization of the localized temperature variance flux into $B\left(x_{i}, R\right)$,

$$
\int_{\mathbb{R}^{2}} \frac{1}{2} \theta^{2}\left(\nabla \phi_{i} \cdot u\right) d x=-\int_{\mathbb{R}^{2}}(u \cdot \nabla \theta) \theta \phi_{i} d x,
$$

which is a consequence of the fact that $u$ is divergence free. An initial formula for the surface-time integrated average flux into $B\left(x_{i}, R\right)$ now follows from (2.4.1),

$$
\begin{aligned}
F_{i} & :=\int_{0}^{2 T} \int_{\mathbb{R}^{2}} \frac{1}{2} \theta^{2}\left(\nabla \phi_{i} \cdot u\right) d x d t \\
& =\int_{0}^{2 T} \int \phi_{i} \theta \Lambda^{\alpha} \theta d x d t-\int_{0}^{2 T} \int \frac{1}{2} \theta^{2}\left(\partial_{t} \phi_{i}\right) d x d t
\end{aligned}
$$

The methodology of Dascaliuc and Grujić (cf. [40]) depends crucially on localized energy estimates which are available in the context of suitable weak solutions for 3D NSE (cf. [20]). Considering the nonlocality introduced by $\Lambda^{\alpha}$, these local estimates are not directly accessible for solutions to SQG when $\alpha<2$, and, consequently, an indirect approach using an extension operator into a third spatial dimension is necessary. Properties of the extension are detailed in [21]; it has previously been applied to the critical and supercritical formulations of SQG in [22] and [39].

The desired extension operator, $L$, is defined in [21] via the initial value problem,

$$
\begin{cases}\nabla \cdot\left(z^{b} \nabla L(f)\right)(x, z)=0 & \text { for }(x, z) \in \mathbb{R}^{2} \times(0, \infty) \\ L(f)(x, 0)=f(x) & \text { for } x \in \mathbb{R}^{2}\end{cases}
$$


where $b=1-\alpha$. The solution $L(f)$ can be represented with a Poissonlike formula and satisfies,

$$
\Lambda^{\alpha} f=\lim _{z \rightarrow 0}\left(-z^{b} \partial_{z}(L f)\right) .
$$

In the critical case this reduces to,

$$
(\Lambda f)(x)=\partial_{\nu}(L f)(x) \text { for } x \in \mathbb{R}^{2} .
$$

Let $\theta$ and $u$ be solutions to (2.4.1) which are smooth on $B\left(x_{0}, 2 R_{0}\right) \times$ $(0,2 T)$. The extension of $\theta$ in the positive $z$-direction is denoted,

$$
\theta^{*}(x, z, t)=L(\theta(\cdot, t))(x, z) .
$$

Our formula for the time-integrated localized flux is obtained similarly to the local energy estimates derived in [22] and [39]. Multiplying (2.4.1) by $\theta \phi_{i}$ where $\phi_{i}$ is a refined cut-off function associated with the ball $B\left(x_{i}, R\right)$ the extension of which in the positive $z$-direction is $\phi_{i}^{*}$, as well as using the properties of $L$, we infer,

$$
\begin{aligned}
F_{i}= & \int_{0}^{2 T} \int_{\mathbb{R}^{2}} \int_{0}^{\infty} \partial_{z}\left(\phi_{i}^{*} \theta^{*} z^{b} \partial_{z} \theta^{*}\right) d z d x d t-\int_{0}^{2 T} \int \frac{1}{2} \theta^{2}\left(\partial_{t} \phi_{i}\right) d x d t \\
= & \int_{0}^{2 T} \int_{\mathbb{R}^{2}} \int_{0}^{\infty} \phi_{i}^{*}\left|\nabla \theta^{*}\right|^{2} z^{b} d z d x d t \\
& +\int_{0}^{2 T} \int_{\mathbb{R}^{2}} \int_{0}^{\infty} \nabla \phi_{i}^{*} \cdot \nabla \theta^{*} z^{b} \theta^{*} d z d x d t \\
& -\int_{0}^{2 T} \int \frac{1}{2} \theta^{2}\left(\partial_{t} \phi_{i}\right) d x d t .
\end{aligned}
$$

\subsubsection{The temperature variance cascade}

Before stating the main result, we identify certain macro-scale averages taken over the time interval $[0,2 T]$ and the ball $B\left(x_{0}, 2 R_{0}\right)$. These are,

$$
\begin{aligned}
\vartheta_{0} & =\frac{1}{T} \int_{0}^{2 T} \frac{1}{R_{0}^{2}} \int \frac{1}{2} \theta^{2} \phi_{0}^{2 \delta-1} d x d t \\
\vartheta_{0}^{*} & =\frac{1}{T} \int_{0}^{2 T} \frac{1}{R_{0}^{2}} \int\left[\int_{0}^{\infty} z^{b} \frac{1}{2} \theta^{* 2} \phi_{0}^{* 2 \delta-1} d z\right] d x d t \\
\varepsilon_{0}^{*} & =\frac{1}{T} \int_{0}^{2 T} \frac{1}{R_{0}^{2}} \int\left[\int_{0}^{\infty} z^{b}\left|\nabla \theta^{*}\right|^{2} \phi_{0}^{*} d z\right] d x d t
\end{aligned}
$$


and have dimensions $l^{2-2 \alpha}, l^{4-3 \alpha}$, and $l^{2-3 \alpha}$ respectively. These dimensions reflect the natural scaling associated with SQG - namely, $\theta_{l}=l^{\alpha-1} \theta\left(l x, l^{\alpha} t\right)$ - and are respected by the following length scale which determines the lower limit of physical scales over which the variance cascade will be shown to persist,

$$
\sigma_{0}=\max \left\{\left(\frac{\vartheta_{0}}{\varepsilon_{0}^{*}}\right)^{1 / \alpha},\left(\frac{\vartheta_{0}^{*}}{\varepsilon_{0}^{*}}\right)^{1 / 2}\right\} .
$$

The main result concerning the temperature variance cascade follows.

Theorem 2.4.2. Fix $K_{1}$ and $K_{2}$ and let

$$
\beta=\min \left\{\left(8 C_{0} K_{1} K_{2}\right)^{-1 / 2},\left(8 C_{0} K_{1} K_{2}\right)^{-1 / \alpha}\right\},
$$

noting that $C_{0}$ is fixed in the definition of our refined cut-off functions. If,

$$
\sigma_{0}<\beta R_{0}
$$

then, for all $R$ satisfying $\frac{1}{\beta} \sigma_{0} \leq R \leq R_{0}$, any $\left(K_{1}, K_{2}\right)$-cover taken at scale $R$ yields an ensemble average satisfying,

$$
\frac{1}{4 K_{1}} \varepsilon_{0}^{*} \leq\langle F\rangle_{R} \leq 4 K_{2} \varepsilon_{0}^{*}
$$

Proof. Invoking the definition of $\left(K_{1}, K_{2}\right)$-covers and the properties of our refined cut-off functions (the fixed constant $C_{0}$ is, in particular, introduced in that context), several relationships are immediate regarding the terms in the formula for $F_{i}$ and the associated ensemble averages. First,

$$
A_{1}:=\left|\frac{1}{n} \sum_{i=1}^{n} \frac{1}{T} \int_{0}^{2 T} \frac{1}{R^{2}} \int_{\mathbb{R}^{2}}\left(\partial_{t} \phi_{i}\right) \frac{\theta^{2}}{2} d x d t\right| \leq \frac{C_{0} K_{2}}{R^{\alpha}} \vartheta_{0} .
$$

Second, using the properties of refined cut-off functions and Young's inequality, we see that,

$$
\begin{aligned}
& \left|\int_{0}^{2 T} \int_{\mathbb{R}^{2}} \int_{0}^{\infty} \nabla \phi_{i}^{*} \cdot \nabla \theta^{*} z^{b} \theta^{*} d z d x d t\right| \\
& \leq \frac{1}{2} \int_{0}^{2 T} \int_{\mathbb{R}^{2}} \int_{0}^{\infty} \phi_{i}^{*}\left|\nabla \theta^{*}\right|^{2} z^{b} d z d x d t \\
& \quad+\frac{C}{R^{2}} \int_{0}^{2 T} \int_{\mathbb{R}^{2}} \int_{0}^{\infty} \frac{1}{2} \theta^{* 2} \phi_{i}^{* 2 \delta-1} z^{b} d z d x d t
\end{aligned}
$$


Ensemble averaging the last term above gives,

$$
A_{2}:=\frac{C}{R^{2}} \frac{1}{n} \sum_{i=1}^{n} \int_{0}^{2 T} \int_{\mathbb{R}^{2}} \int_{0}^{\infty} \frac{1}{2} \theta^{* 2} \phi_{i}^{* 2 \delta-1} z^{b} d z d x d t \leq \frac{C_{0} K_{2}}{R^{2}} \vartheta_{0}^{*} .
$$

As the involved integrands are positive, direct comparison and the definition of $\left(K_{1}, K_{2}\right)$-covers yields the following interpolative estimate,

$$
\frac{1}{K_{1}} \varepsilon_{0}^{*} \leq \frac{1}{n} \sum_{i=1}^{n} \frac{1}{T} \int_{0}^{2 T} \frac{1}{R^{2}} \iint_{0}^{\infty} z^{b}\left|\nabla \theta^{*}\right|^{2} \phi_{i}^{*} d z d x d t \leq K_{2} \varepsilon_{0}^{*}
$$

Returning to our formula for $F_{i}$, taking the ensemble average and using the above facts reveals that,

$$
\begin{aligned}
\langle F\rangle_{R} & \leq \frac{3}{2} \frac{1}{n} \sum_{i=1}^{n} \frac{1}{T} \int_{0}^{2 T} \frac{1}{R^{2}} \iint_{0}^{\infty} z^{b}\left|\nabla \theta^{*}\right|^{2} \phi_{i}^{*} d z d x d t+A_{1}+A_{2} \\
& \leq 2 K_{2} \varepsilon_{0}^{*}+\frac{C_{0} K_{2}}{R^{2}} \vartheta_{0}^{*}+\frac{C_{0} K_{2}}{R^{\alpha}} \vartheta_{0},
\end{aligned}
$$

and,

$$
\begin{aligned}
\langle F\rangle_{R} & \geq \frac{1}{2} \frac{1}{n} \sum_{i=1}^{n} \frac{1}{T} \int_{0}^{2 T} \frac{1}{R^{2}} \iint_{0}^{\infty} z^{b}\left|\nabla \theta^{*}\right|^{2} \phi_{i}^{*} d z d x d t-A_{1}-A_{2} \\
& \geq \frac{1}{2 K_{1}} \varepsilon_{0}^{*}-\frac{C_{0} K_{2}}{R^{2}} \vartheta_{0}^{*}-\frac{C_{0} K_{2}}{R^{\alpha}} \vartheta_{0} .
\end{aligned}
$$

Recalling the definition of $\beta$ and the assumption that $\sigma_{0} / \beta \leq R \leq R_{0}$, we conclude our proof as we have shown that,

$$
\frac{1}{4 K_{1}} \varepsilon_{0}^{*} \leq\langle F\rangle_{R} \leq 4 K_{2} \varepsilon_{0}^{*}
$$

Scale-locality of the temperature variance flux, i.e. that the average flux at a scale $R$ lying in the inertial range is most strongly correlated with the average fluxes at nearby scales, follows directly from Theorem 2.4 .2 and, moreover, this correlation propagates exponentially along the dyadic scale (this is expected in SQG turbulence where large scale 
strain plays less of a role than in hydrodynamic turbulence, cf. [92]). We adopt the notation,

$$
G_{i}=\frac{1}{T} \int_{0}^{2 T} \int \frac{1}{2} \theta^{2} u \cdot \nabla \phi_{i} d x d t=R^{2} F_{i},
$$

for the time averaged localized temperature variance flux into the ball $B\left(x_{i}, R\right)$ and $\langle G\rangle_{R}$ for the associated ensemble average over a $\left(K_{1}, K_{2}\right)$ cover taken at scale $R$. The following corollary affirms the scale-locality of the temperature variance flux across the range of scales on which the cascade persists. The proof is omitted.

Corollary 2.4.3. If the premises of Theorem 2.4.2 are satisfied and $\frac{1}{\beta} \sigma_{0} \leq r, R \leq R_{0}$, then,

$$
\frac{1}{16 K_{1} K_{2}}\left(\frac{r}{R}\right)^{2} \leq \frac{\langle G\rangle_{r}}{\langle G\rangle_{R}} \leq 16 K_{1} K_{2}\left(\frac{r}{R}\right)^{2} .
$$

To see the exponential propagation along the dyadic scale, observe that,

$$
\frac{1}{16 K_{1} K_{2}} 2^{2 k} \leq \frac{\langle G\rangle_{r}}{\langle G\rangle_{R}} \leq 16 K_{1} K_{2} 2^{2 k}
$$

where $r=2^{k} R$ for some integer $k$. 


\section{Chapter 3}

\section{Uniform analyticity radii and anisotropic diffusion}

\subsection{Introduction}

The uniform analyticity radius associated with a fluid's velocity field has been connected to small scales of spatial complexity by the observation that analytic functions have Fourier transforms which fall off exponentially at high wavenumbers. The implication is that the behavior at small physical scales is dampened so long as a solution is analytic and this motivates a connection between the uniform analyticity radii of a fluid and the dissipative scale - i.e. the scale at which viscous forces dominate inertial effects and inertial range transport dynamics break down as energy is lost in the form of heat. A careful description of this is given in [55] (see, in particular, Chapter 6.3.2) and there has subsequently been a mathematical interest in providing sharp lower bounds for this length scale directly from the governing equations. A pioneering work in this area is due to C. Foias and R. Temam (cf. [54]) using Gevrey space techniques. This approach has recently been revisited using modern Fourier analysis techniques (cf. $[10,4]$ ) and also applied to study the decay of the analyticity radii of solutions to 3D Euler with real analytic initial data (cf. [79, 78, 80]). An alternative approach was developed in $[62,63,76]$ using $L^{p}$ space techniques $(3<p<\infty)$ and, later, extended to the case of mild solutions with initial data in $L^{\infty}\left(\mathbb{R}^{3}\right)$ (cf. [64]; we contain an extension of this argument to 3D MHD in Section 3.2).

Existing approaches for solutions of to NSE require that the forcing 
term is itself spatially analytic and, moreover, has a uniform analyticity radius $\lambda_{f}$, i.e. it is the restriction of a function that is analytic on the domain $\left\{x+i y \in \mathbb{C}^{3}:|y|<\lambda_{f}\right\}$. Because the forcing has a local influence on the evolution of a flow it is natural to investigate whether or not the dependence of the velocity field's analyticity radius on the analyticity radius of the forcing term can be localized. An affirmative answer can be formulated (see Section 3.4) by adapting a technique developed in [63] to estimate the analyticity radius of a non-linear heat equation defined on a bounded domain. The contrast between the work of [63] and our own result lies in the fact that the non-linear contributions are considerably more complicated in NSE and the approximation scheme constructed in [63], when adapted to solutions of 3D NSE, introduces non-linear terms which are not depleted by incompressibility. Although we work with a similar scheme our estimates are carried out using mild solutions in a fashion motivated by [72]. The benefit here lies in the approaches elegance as well as illustrating how Kato's method can be extended to non-linear perturbations of heat equations possessing second order linear terms.

The uniform analyticity radius has also been connected to the regularity of a solution to 3D NSE (cf. [61]). In particular, the analyticity properties of a solution can be connected to a physically and numerically apparent anisotropic diffusion which leads to one-dimensional sparseness of the region of high spatial complexity in such a way to prevent the formation of a singularity. Essentially, if the transverse length scales of the super-level sets of the vorticity decay at a faster rate than the uniform analyticity radius then a regularity criteria specified in [61] is triggered. In 3D MHD turbulence, a strong anisotropic effect is also apparent in the evolution of current sheets [8]. The second result of this chapter establishes a similar regularity criteria for solutions to 3D MHD and is contained in Section 3.3.

\subsection{Sharp lower bounds on the analyticity radii of solutions to $3 \mathrm{D}$ MHD with $L^{\infty}$ initial data}

We are interested in the 3D MHD system with bounded initial data and its analytic extension into $\mathbb{C}^{3}$. The $\mathbb{R}^{3}$ system is as stated in prior 
sections but we presently substitute the labels $U$ and $B$ for $u$ and $b$ since the latter will be associated with complex extensions of the former fields throughout this chapter.

Let $\lambda>0$ and recall that the fundamental solution of the homogeneous heat equations - i.e. $\partial_{t} f-\lambda \Delta f=0$ - is given (for $t>0$ ) by,

$$
G_{\lambda}(x, t)=\frac{1}{(2 \pi \lambda t)^{D / 2}} e^{\frac{-|x|^{2}}{4 \lambda t}}=G(x, \lambda t)
$$

Using the Gaussian kernel we have the following definition of mild solutions to MHD.

Definition 3.2.1. Let $U_{0}, B_{0} \in L^{\infty}\left(\mathbb{R}^{D}\right)$, both divergence free. The functions $U, B \in C\left((0, T), L^{\infty}\left(\mathbb{R}^{D}\right)\right)$ comprise a mild solution to $M H D$ on the time interval $[0, T)$ if, for every $(x, t) \in \mathbb{R}^{D} \times(0, T)$, they satisfy,

$$
\begin{aligned}
U_{k}(x, t)= & \int_{\mathbb{R}^{D}} G_{\nu}(x-w, t) U_{0 k}(w) d w \\
& -\int_{0}^{t} \int_{\mathbb{R}^{D}} \partial_{j} G_{\nu}(x-w, t-s) U_{j}(w, s) U_{k}(w, s) d w d s \\
& +\int_{0}^{t} \int_{\mathbb{R}^{D}} \partial_{j} G_{\nu}(x-w, t-s) B_{j}(w, s) B_{k}(w, s) d w d s \\
& -\int_{0}^{t} \int_{\mathbb{R}^{D}} \nabla G_{\nu}(x-w, t-s) \Pi(w, s) d w d s,
\end{aligned}
$$

and,

$$
\begin{aligned}
B_{k}(x, t)= & \int_{\mathbb{R}^{D}} G_{\mu}(x-w, t) B_{0 k}(w) d w \\
& -\int_{0}^{t} \int_{\mathbb{R}^{D}} \partial_{j} G_{\mu}(x-w, t) U_{j}(w, s) B_{k}(w, s) d w d s \\
& +\int_{0}^{t} \int_{\mathbb{R}^{D}} \partial_{j} G_{\mu}(x-w, t) B_{j}(w, s) U_{k}(w, s) d w d s
\end{aligned}
$$

where,

$$
\Delta \Pi=-\partial_{j} \partial_{k}\left(U_{j} U_{k}-B_{j} B_{k}\right)
$$


We will extract information about a mild solution via a standard iterative argument. Consider the recursively defined scheme,

$$
\begin{aligned}
& U^{(0)}=\Pi^{(0)}=B^{(0)}=0 \\
& \partial_{t} U^{(n)}-\nu \Delta U^{(n)}=-U^{(n-1)} \cdot \nabla U^{(n-1)}+B^{(n-1)} \cdot \nabla B^{(n-1)}-\nabla \Pi^{(n-1)} \\
& \partial_{t} B^{(n)}-\mu \Delta B^{(n)}=-U^{(n-1)} \cdot \nabla B^{(n-1)}+B^{(n-1)} \cdot \nabla U^{(n-1)} \\
& U^{(n)}(x, 0)=U_{0}(x) ; \quad B^{(n)}(x, 0)=B_{0}(x) \\
& \Delta \Pi^{(n)}=-\partial_{j} \partial_{k}\left(U_{j}^{(n)} U_{k}^{(n)}-B_{j}^{(n)} B_{k}^{(n)}\right) .
\end{aligned}
$$

When $n=0$ the approximating fields are obtained by solving homogeneous heat equations and Poisson equations and, for subsequent values of $n$, are solutions to inhomogeneous heat or Poisson equations and existence is thus guaranteed via classical results which also ensure the smoothness properties of previous iterates are inherited. We will additionally show that certain uniform bounds are satisfied for all values of $n$ and for this we need several lemmas. Note that, up to the incorporation of a constant $\lambda$, these are identical to their counterparts in [77] and employ facts from $[101,103]$. Proofs are consequently omitted.

Lemma 3.2.2. If $f \in L^{\infty}$ then there exists a unique solution, $\pi \in$ $B M O$, to the problem,

$$
-\Delta \pi=\partial_{j} \partial_{k} f
$$

Furthermore,

$$
\|\pi\|_{B M O} \leq C\|f\|_{\infty}
$$

Lemma 3.2.3. For $T>0$ and $f_{j} \in L^{\infty}(0, T, B M O)$ where $j=$ $1, \ldots, D$, we have the inequality,

$$
\left|\int_{0}^{t} \int_{\mathbb{R}^{D}} \partial_{j} G_{\lambda}(x-y, t-s) f_{j}(y, s) d y d s\right| \leq C_{\lambda} \sqrt{T}\|f\|_{L^{\infty}(0, T, B M O)},
$$

where $C_{\lambda}$ is a constant depending on $\lambda$ and $D$.

We turn now to an inductive argument which we use to establish uniform $L^{\infty}\left(\mathbb{R}^{3} \times(0, T)\right)$ estimates for an appropriate time $T>0$. Hypothesize that $U^{(n-1)}, B^{(n-1)}$, and $\Pi^{(n-1)}$ all satisfy the scheme distributionally and that the first two vector fields are in $L^{\infty}\left(\mathbb{R}^{3} \times(0, T)\right)$. Then, $U^{(n)}$ and $B^{(n)}$ exist and, moreover, can be expressed via Duhamel's procedure while the existence of $\Pi^{(n)}$ follows from Lemma 3.2.2. More can be said about these solutions. 
Lemma 3.2.4. Given $U_{0}, B_{0} \in L^{\infty}$ there exists $T_{1}>0$ so that if $T \leq T_{1}$ then, for all $n \in \mathbb{N}$ and all pairs $(x, t) \in \mathbb{R}^{D} \times[0, T)$, we have,

$$
\begin{aligned}
& \left\|U^{(n)}\right\|_{L^{\infty}\left([0, T) \times \mathbb{R}^{D}\right)}+\left\|B^{(n)}\right\|_{L^{\infty}\left([0, T) \times \mathbb{R}^{D}\right)} \\
& \leq 2 C_{1}\left(\left\|U_{0}\right\|_{L^{\infty}\left([0, T) \times \mathbb{R}^{D}\right)}+\left\|B_{0}\right\|_{L^{\infty}\left([0, T) \times \mathbb{R}^{D}\right)}\right),
\end{aligned}
$$

where $C_{1}$ is independent of $n$ and depends on $\mu, \nu$, and $D$, and,

$$
T_{1}=\frac{1}{4\left(\left\|U_{0}\right\|_{L^{\infty}\left(\mathbb{R}^{D}\right)}+\left\|B_{0}\right\|_{L^{\infty}\left(\mathbb{R}^{D}\right)}\right)^{2}} .
$$

Proof. Using the formulas for $U^{(n)}$ and $B^{(n)}$ obtained via Duhamel's procedure, an integration by parts yields,

$$
\begin{aligned}
U^{(n)}(x, t)= & \int_{\mathbb{R}^{D}} G_{\nu}(x-w, t) U_{0}(x) d w \\
& -\int_{0}^{t} \int_{\mathbb{R}^{D}} \partial_{j} G_{\nu}(x-w, t-s) U_{j}^{(n-1)}(w, s) U^{(n-1)}(w, s) d w d s \\
& +\int_{0}^{t} \int_{\mathbb{R}^{D}} \partial_{j} G_{\nu}(x-w, t-s) B_{j}^{(n-1)}(w, s) B^{(n-1)}(w, s) d w d s \\
& -\int_{0}^{t} \int_{\mathbb{R}^{D}} \nabla G_{\nu}(x-w, t-s) \Pi^{(n-1)}(w, s) d w d s
\end{aligned}
$$

and,

$$
\begin{aligned}
B^{(n)}(x, t)= & \int_{\mathbb{R}^{D}} G_{\mu}(x-w, t) B_{0}(x) d w \\
& -\int_{0}^{t} \int_{\mathbb{R}^{D}} \partial_{j} G_{\mu}(x-w, t-s) U_{j}^{(n-1)}(w, s) B^{(n-1)}(w, s) d w d s \\
& +\int_{0}^{t} \int_{\mathbb{R}^{D}} \partial_{j} G_{\mu}(x-w, t-s) B_{j}^{(n-1)}(w, s) U^{(n-1)}(w, s) d w d s .
\end{aligned}
$$

Applying Lemma 3.2.3 to each summand above (and noting that the products of approximates from the scheme appearing in the integrands are in $B M O$ since $\left.L^{\infty} \subset B M O\right)$ grants that,

$$
\begin{aligned}
\left|U^{(n)}\right| \leq & C_{\nu}|| U_{0}\left\|_{L^{\infty}\left(\mathbb{R}^{D}\right)}+C_{\nu} \sqrt{T}\right\| U^{(n-1)} \|_{L^{\infty}\left((0, T) \times \mathbb{R}^{D}\right)}^{2} \\
& +C_{\nu} \sqrt{T}\left\|B^{(n-1)}\right\|_{L^{\infty}\left((0, T) \times \mathbb{R}^{D}\right)}^{2}+C_{\nu} \sqrt{T}\left\|\Pi^{(n-1)}\right\|_{L^{\infty}((0, T), B M O)},
\end{aligned}
$$


and,

$$
\begin{aligned}
\left|B^{(n)}\right| \leq & C_{\mu}|| B_{0} \|_{L^{\infty}\left(\mathbb{R}^{D}\right)} \\
& +2 C_{\mu} \sqrt{T}\left\|U^{(n-1)}\right\|_{L^{\infty}\left((0, T) \times \mathbb{R}^{D}\right)}\left\|B^{(n-1)}\right\|_{L^{\infty}\left((0, T) \times \mathbb{R}^{D}\right)} .
\end{aligned}
$$

Also, in light of Lemma 3.2.2 we have that,

$$
\begin{aligned}
& \left\|\Pi^{(n-1)}\right\|_{L^{\infty}((0, T), B M O)} \\
& \leq C\left(\left\|U^{(n-1)}\right\|_{L^{\infty}\left((0, T) \times \mathbb{R}^{D}\right)}^{2}+\left\|B^{(n-1)}\right\|_{L^{\infty}\left((0, T) \times \mathbb{R}^{D}\right)}^{2}\right),
\end{aligned}
$$

where $C$ is the constant appearing in Lemma 3.2.2. Letting $C_{1}$ be appropriately large we obtain the estimates,

$$
\begin{aligned}
\left|U^{(n)}\right|+\left|B^{(n)}\right| \leq & C_{1}\left(\left\|U_{0}\right\|_{L^{\infty}\left(\mathbb{R}^{D}\right)}+\left\|B_{0}\right\|_{L^{\infty}\left(\mathbb{R}^{D}\right)}\right) \\
& +C_{1} \sqrt{T}\left(\left\|U^{(n-1)}\right\|_{L^{\infty}\left((0, T) \times \mathbb{R}^{D}\right)}^{2}\right. \\
& +2\left\|U^{(n-1)}\right\|_{L^{\infty}\left((0, T) \times \mathbb{R}^{D}\right)}\left\|B^{(n-1)}\right\|_{L^{\infty}\left((0, T) \times \mathbb{R}^{D}\right)} \\
& \left.+\left\|B^{(n-1)}\right\|_{L^{\infty}\left((0, T) \times \mathbb{R}^{D}\right)}^{2}\right),
\end{aligned}
$$

which factors to give a bound independent of $n, x$, and $t$, i.e.,

$$
\begin{aligned}
& \left|U^{(n)}\right|+\left|B^{(n)}\right| \\
& \leq C_{1}\left(\left\|U_{0}\right\|_{L^{\infty}\left(\mathbb{R}^{D}\right)}+\left\|B_{0}\right\|_{L^{\infty}\left(\mathbb{R}^{D}\right)}\right) \\
& \quad+C_{1} \sqrt{T}\left(\left\|U^{(n-1)}\right\|_{L^{\infty}\left((0, T) \times \mathbb{R}^{D}\right)}+\left\|B^{(n-1)}\right\|_{L^{\infty}\left((0, T) \times \mathbb{R}^{D}\right)}\right)^{2} .
\end{aligned}
$$

Setting,

$$
T_{1}=\frac{1}{4\left(\left\|U_{0}\right\|_{L^{\infty}\left(\mathbb{R}^{D}\right)}+\left\|B_{0}\right\|_{L^{\infty}\left(\mathbb{R}^{D}\right)}\right)^{2}},
$$

and assuming that $0<T \leq T_{1}$ we obtain for $n=1$ that,

$$
\left|U^{(1)}\right|+\left|B^{(1)}\right| \leq 2 C_{1}\left(|| U_{0}\left\|_{L^{\infty}\left(\mathbb{R}^{D}\right)}+\right\| B_{0} \|_{L^{\infty}\left(\mathbb{R}^{D}\right)}\right),
$$

and, by induction, this extends to all $n \in \mathbb{N}$, i.e. we have,

$$
\left|U^{(n)}\right|+\left|B^{(n)}\right| \leq 2 C_{1}\left(|| U_{0}\left\|_{L^{\infty}\left(\mathbb{R}^{D}\right)}+\right\| B_{0} \|_{L^{\infty}\left(\mathbb{R}^{D}\right)}\right) .
$$

The existence of mild solutions as limits of approximation scheme elements follows from a typical contractivity argument. 
Theorem 3.2.5. Given $U_{0}, B_{0} \in L^{\infty}\left(\mathbb{R}^{D}\right)$ there exists $T_{2}>0$ so that, for $T<T_{2}$, a mild solution to MHD exists on $[0, T]$ with,

$$
T_{2}=\frac{1}{\left(4 C_{1} C_{2}\left(\left\|U_{0}\right\|_{L^{\infty}\left(\mathbb{R}^{D}\right)}+\left\|B_{0}\right\|_{L^{\infty}\left(\mathbb{R}^{D}\right)}\right)\right)^{2}},
$$

where $C_{2}$ is a constant depending on $\mu, \nu$, and D. Furthermore, the mild solution obtained in this fashion is unique.

Proof. We assume that $U_{0}, B_{0} \in L^{\infty}\left(\mathbb{R}^{D}\right)$. Our first step will be to appropriately restrict $T$ in order to establish the inequality,

$$
\begin{aligned}
& \left\|U^{(n+1)}-U^{(n)}\right\|_{L^{\infty}\left((0, T) \times \mathbb{R}^{D}\right)}+\left\|B^{(n+1)}-B^{(n)}\right\|_{L^{\infty}\left((0, T) \times \mathbb{R}^{D}\right)} \\
& \leq \alpha\left(\left\|U^{(n)}-U^{(n-1)}\right\|_{L^{\infty}\left((0, T) \times \mathbb{R}^{D}\right)}+\left\|B^{(n)}-B^{(n-1)}\right\|_{L^{\infty}\left((0, T) \times \mathbb{R}^{D}\right)}\right),
\end{aligned}
$$

for some $\alpha \in(0,1)$. Let $\mathcal{U}^{(n+1)}=U^{(n+1)}-U^{(n)}$ and $\mathcal{B}^{(n+1)}=B^{(n+1)}-$ $B^{(n)}$. By applying Duhamel's principle and an integration by parts we obtain formulas for $\mathcal{U}^{(n+1)}$ and $\mathcal{B}^{(n+1)}$. The bounding procedure from Lemma 3.2.4 ultimately leads to the estimate,

$$
\begin{aligned}
& \left|\mathcal{U}^{(n+1)}\right|+\left|\mathcal{B}^{(n+1)}\right| \\
& \leq C \sqrt{T}|| \mathcal{U}^{(n)} \|_{L^{\infty}\left((0, T) \times \mathbb{R}^{D}\right)} \\
& \quad \cdot\left(\left\|U^{(n)}\right\|_{L^{\infty}\left((0, T) \times \mathbb{R}^{D}\right)}+\left\|B^{(n)}\right\|_{L^{\infty}\left((0, T) \times \mathbb{R}^{D}\right)}\right. \\
& \left.\quad+\left\|U^{(n-1)}\right\|_{L^{\infty}\left((0, T) \times \mathbb{R}^{D}\right)}+\left\|B^{(n-1)}\right\|_{L^{\infty}\left((0, T) \times \mathbb{R}^{D}\right)}\right) \\
& +C \sqrt{T}\left\|\mathcal{B}^{(n)}\right\|_{L^{\infty}\left((0, T) \times \mathbb{R}^{D}\right)} \\
& \quad \cdot\left(\left\|U^{(n)}\right\|_{L^{\infty}\left((0, T) \times \mathbb{R}^{D}\right)}+\left\|B^{(n)}\right\|_{L^{\infty}\left((0, T) \times \mathbb{R}^{D}\right)}\right. \\
& \left.\quad+\left\|U^{(n-1)}\right\|_{L^{\infty}\left((0, T) \times \mathbb{R}^{D}\right)}+\left\|B^{(n-1)}\right\|_{L^{\infty}\left((0, T) \times \mathbb{R}^{D}\right)}\right) \\
& \leq C \\
& \quad \sqrt{T} 4 C_{1}\left(\left\|U_{0}\right\|_{L^{\infty}\left((0, T) \times \mathbb{R}^{D}\right)}+\left\|B_{0}\right\|_{L^{\infty}\left((0, T) \times \mathbb{R}^{D}\right)}\right) \\
& \quad \cdot\left(\left\|\mathcal{U}^{(n)}\right\|_{L^{\infty}\left((0, T) \times \mathbb{R}^{D}\right)}+\left\|\mathcal{B}^{(n)}\right\|_{L^{\infty}\left((0, T) \times \mathbb{R}^{D}\right)}\right) .
\end{aligned}
$$

Set $C_{2}$ to be the value of the constant $C$ as it is appears above,

$$
T_{2}=\frac{1}{\left(4 C_{1} C_{2}\left(\left\|U_{0}\right\|_{L^{\infty}\left(\mathbb{R}^{3}\right)}+\left\|B_{0}\right\|_{L^{\infty}\left(\mathbb{R}^{3}\right)}\right)\right)^{2}} .
$$


Assuming $T=\alpha T_{2}$ where $\alpha \in(0,1)$, we see that,

$$
\begin{aligned}
& \left\|\mathcal{U}^{(n+1)}\right\|_{L^{\infty}\left((0, T) \times \mathbb{R}^{D}\right)}+\left\|\mathcal{B}^{(n+1)}\right\|_{L^{\infty}\left((0, T) \times \mathbb{R}^{D}\right)} \\
& \leq \alpha\left(\left\|\mathcal{U}^{(n)}\right\|_{L^{\infty}\left((0, T) \times \mathbb{R}^{D}\right)}+\left\|\mathcal{B}^{(n)}\right\|_{L^{\infty}\left((0, T) \times \mathbb{R}^{D}\right)}\right) .
\end{aligned}
$$

Consequently we obtain a bound which diminishes as $n$ escapes, namely,

$$
\begin{aligned}
& \left\|\mathcal{U}^{(n)}\right\|_{L^{\infty}\left((0, T) \times \mathbb{R}^{D}\right)}+\left\|\mathcal{B}^{(n)}\right\|_{L^{\infty}\left((0, T) \times \mathbb{R}^{D}\right)} \\
& \leq \alpha^{n-1}\left(\left\|\mathcal{U}^{(1)}\right\|_{L^{\infty}\left((0, T) \times \mathbb{R}^{D}\right)}+\left\|\mathcal{B}^{(1)}\right\|_{L^{\infty}\left((0, T) \times \mathbb{R}^{D}\right)}\right),
\end{aligned}
$$

and a simple convergence argument applies. Noting that $\alpha^{n-1}$ vanishes as $n \rightarrow \infty$, the above bound ensures that both $\left\{U^{(n)}\right\}$ and $\left\{B^{(n)}\right\}$ are pointwise Cauchy and so we obtain pointwise limits $U$ and $B$. The dominated convergence theorem (the dominator is the respective Gaussian kernel, scaled) then verifies that $U$ and $B$ are indeed mild solutions.

Uniqueness follows from the fact that for two mild solutions $U, B$ and $\tilde{U}, \tilde{B}$, we have,

$$
\begin{aligned}
& \|U-\tilde{U}\|_{\infty}+\|B-\tilde{B}\|_{\infty} \\
& \leq 4 C \sqrt{\tilde{T}}\left(\|U-\tilde{U}\|_{\infty}+\|B-\tilde{B}\|_{\infty}\right) \\
& \quad \cdot\left(\|U\|_{\infty}+\|\tilde{U}\|_{\infty}+\|B\|_{\infty}+\|\tilde{B}\|_{\infty}\right) .
\end{aligned}
$$

Then, taking $\tilde{T}$ small enough so that,

$$
4 C \sqrt{\tilde{T}}\left(\|U\|_{\infty}+\|\tilde{U}\|_{\infty}+\|B\|_{\infty}+\|\tilde{B}\|_{\infty}\right)<1,
$$

we obtain $U=\tilde{U}$ and $B=\tilde{B}$ on $[0, \tilde{T}]$. This argument is iterated to obtain the conclusion across the entire time interval $[0, T]$.

Slightly more can be said and we include a corollary which will be used later to minimize the geometric relevance of the magnetic field in our regularity result.

Corollary 3.2.6. Suppose that $U$ and $B$ are the mild solutions obtained above to $M H D$ on the time interval $[0, T)$ (so, $U_{0}, B_{0} \in L^{\infty}\left(\mathbb{R}^{D}\right)$ and $\left.T<T_{2}\right)$. Then, there exists $T_{3}>0$ so that

$$
\|B\|_{L^{\infty}\left(\left(0, T_{3}\right) \times \mathbb{R}^{D}\right)} \leq 2 C_{3}\left\|B_{0}\right\|_{L^{\infty}\left(\mathbb{R}^{D}\right)}
$$

where $C_{3}$ is a constant depending on $D$ and $\mu$. 
Proof. In the proof of Lemma 3.2.4 we saw the bound,

$$
\begin{aligned}
\left|B^{(n)}\right| \leq & C_{\mu}|| B_{0} \|_{L^{\infty}\left(\mathbb{R}^{D}\right)} \\
& +2 C_{\mu} \sqrt{T}|| U^{(n-1)}\left\|_{L^{\infty}\left((0, T) \times \mathbb{R}^{D}\right)}|| B^{(n-1)}\right\|_{L^{\infty}\left((0, T) \times \mathbb{R}^{D}\right)} .
\end{aligned}
$$

Set

$$
T_{3}=\min \left\{T, \frac{1}{16 C_{\mu}^{2}\|U\|_{L^{\infty}\left((0, T) \times \mathbb{R}^{D}\right)}^{2}}\right\} .
$$

Then, for any $(t, x) \in\left[0, T_{3}\right) \times \mathbb{R}^{D}$,

$$
\begin{aligned}
\left|B^{(n)}(t, x)\right| \leq & C_{\mu}\left\|B_{0}\right\|_{L^{\infty}\left(\mathbb{R}^{D}\right)} \\
& +\frac{\left\|U^{(n-1)}\right\|_{L^{\infty}\left(\left(0, T_{3}\right) \times \mathbb{R}^{D}\right)}}{2\|U\|_{L^{\infty}\left(\left(0, T_{3}\right) \times \mathbb{R}^{D}\right)}}\left\|B^{(n-1)}\right\|_{L^{\infty}\left(\left(0, T_{3}\right) \times \mathbb{R}^{D}\right)} .
\end{aligned}
$$

Taking limits and setting $C_{3}=C_{\mu}$ we see,

$$
\|B\|_{L^{\infty}\left(\left(0, T_{3}\right) \times \mathbb{R}^{D}\right)} \leq C_{3}\left\|B_{0}\right\|_{L^{\infty}\left(\mathbb{R}^{D}\right)}+\frac{1}{2}\|B\|_{L^{\infty}\left(\left(0, T_{3}\right) \times \mathbb{R}^{D}\right)} .
$$

Having established needed properties of the real-variable system we proceed to adapt the arguments of [63] and [64] from the case of NSE in $D$ dimensions to that of 3D MHD. Essential to later work will be a uniform bound on the analytic extensions of mild solutions to a certain complex domain. We restrict our attention to highlighting this result while appealing to the existing literature to fill in technical omissions.

Recalling the approximation scheme for the real variable discussion, let $u^{(n)}+i v^{(n)}, b^{(n)}+i c^{(n)}$, and $\pi^{(n)}+i \rho^{(n)}$ be the analytic extensions of $U^{(n)}, B^{(n)}$ and $\Pi^{(n)}$ respectively. Real analyticity of the $n$-th approximation is a consequence of real analyticity of solutions of the heat and Poisson equations. Substituting these extensions into the scheme and subsequently isolating real and imaginary parts reveals that the analytic 
extensions of the real-variable scheme satisfy the evolution equations,

$$
\begin{aligned}
\partial_{t} u^{(n)}-\nu \Delta u^{(n)}= & -\left(u^{(n-1)} \cdot \nabla\right) u^{(n-1)}+\left(v^{(n-1)} \cdot \nabla\right) v^{(n-1)} \\
& +\left(b^{(n-1)} \cdot \nabla\right) b^{(n-1)}-\left(c^{(n-1)} \cdot \nabla\right) c^{(n-1)}-\nabla \pi^{(n-1)} \\
\partial_{t} v^{(n)}-\nu \Delta v^{(n)}= & -\left(u^{(n-1)} \cdot \nabla\right) v^{(n-1)}-\left(v^{(n-1)} \cdot \nabla\right) u^{(n-1)} \\
& +\left(b^{(n-1)} \cdot \nabla\right) c^{(n-1)}+\left(c^{(n-1)} \cdot \nabla\right) b^{(n-1)}-\nabla \rho^{(n-1)} \\
\partial_{t} b^{(n)}-\mu \Delta b^{(n)}= & -\left(u^{(n-1)} \cdot \nabla\right) b^{(n-1)}+\left(v^{(n-1)} \cdot \nabla\right) c^{(n-1)} \\
& +\left(b^{(n-1)} \cdot \nabla\right) u^{(n-1)}-\left(c^{(n-1)} \cdot \nabla\right) v^{(n-1)} \\
\partial_{t} c^{(n)}-\mu \Delta c^{(n)}= & -\left(v^{(n-1)} \cdot \nabla\right) b^{(n-1)}-\left(u^{(n-1)} \cdot \nabla\right) c^{(n-1)} \\
& +\left(b^{(n-1)} \cdot \nabla\right) v^{(n-1)}+\left(c^{(n-1)} \cdot \nabla\right) u^{(n-1)}
\end{aligned}
$$

as well as the kinematic equations,

$$
\begin{aligned}
\Delta \pi^{(n)} & =-\partial_{j} \partial_{k}\left(u_{j}^{(n)} u_{k}^{(n)}-v_{j}^{(n)} v_{k}^{(n)}\right)+\partial_{j} \partial_{k}\left(b_{j}^{(n)} b_{k}^{(n)}-c_{j}^{(n)} c_{k}^{(n)}\right) \\
\Delta \rho^{(n)} & =-\partial_{j} \partial_{k}\left(u_{j}^{(n)} v_{k}^{(n)}+u_{k}^{(n)} v_{j}^{(n)}\right)+\partial_{j} \partial_{k}\left(b_{j}^{(n)} c_{k}^{(n)}+b_{k}^{(n)} c_{j}^{(n)}\right) .
\end{aligned}
$$

Letting $y=\alpha t$ with $\alpha \in \mathbb{R}^{n}$ and $t \geq 0$ allows us to determine a sharp lower bound for the analyticity radius in terms of $t$. Substituting these terms into the approximation scheme, omitting for brevity the terms associated with the total pressure which are just solutions of Poisson equations analogous to the real variable scheme, we obtain that,

$$
\begin{aligned}
\partial_{t} u^{(n)}-\nu \Delta u^{(n)}= & \alpha_{j}\left(\partial_{j} v^{(n)}\right)-\left(u^{(n-1)} \cdot \nabla\right) u^{(n-1)}+\left(v^{(n-1)} \cdot \nabla\right) v^{(n-1)} \\
& -\left(b^{(n-1)} \cdot \nabla\right) b^{(n-1)}+\left(c^{(n-1)} \cdot \nabla\right) c^{(n-1)}+\nabla \pi^{(n-1)} \\
\partial_{t} v^{(n)}-\nu \Delta v^{(n)}= & -\alpha_{j}\left(\partial_{j} u^{(n)}\right)-\left(u^{(n-1)} \cdot \nabla\right) v^{(n-1)}-\left(v^{(n-1)} \cdot \nabla\right) u^{(n-1)} \\
& +\left(b^{(n-1)} \cdot \nabla\right) c^{(n-1)}+\left(c^{(n-1)} \cdot \nabla\right) b^{(n-1)}+\nabla \rho^{(n-1)} \\
\partial_{t} b^{(n)}-\mu \Delta b^{(n)}= & -\alpha_{j}\left(\partial_{j} c^{(n)}\right)-\left(u^{(n-1)} \cdot \nabla\right) b^{(n-1)}+\left(v^{(n-1)} \cdot \nabla\right) c^{(n-1)} \\
& +\left(b^{(n-1)} \cdot \nabla\right) u^{(n-1)}-\left(c^{(n-1)} \cdot \nabla\right) v^{(n-1)} \\
\partial_{t} c^{(n)}-\mu \Delta c^{(n)}= & -\alpha_{j}\left(\partial_{j} b^{(n)}\right)-\left(v^{(n-1)} \cdot \nabla\right) b^{(n-1)}-\left(u^{(n-1)} \cdot \nabla\right) c^{(n-1)} \\
& +\left(b^{(n-1)} \cdot \nabla\right) v^{(n-1)}+\left(c^{(n-1)} \cdot \nabla\right) u^{(n-1)} \\
u^{(n)}(x, 0,0)= & U_{0}(x) \\
b^{(n)}(x, 0,0)= & B_{0}(x) \\
v^{(n)}(x, 0,0)= & c^{(n)}(x, 0,0)=0
\end{aligned}
$$


Note that these systems evolve from the initial data $U_{0}$ and $B_{0}$ and are thus meaningfully defined even when these fields are not realanalytic. Bounds on the sum of the $L^{\infty}\left([0, T) \times \mathbb{R}^{n}\right)$ norms of $u^{(n)}$, $v^{(n)}, b^{(n)}$, and $c^{(n)}$ for appropriate values of $T$ (denote by $F_{n}$ the set $\left.\left\{u^{(n)}, v^{(n)}, b^{(n)}, c^{(n)}\right\}\right)$ are obtained in a familiar fashion. Duhamel's Principle and a subsequent integration by parts yields formulas for each approximate in $F_{n}$. These approximates can then be bounded using the methods seen in the proof of Lemma 3.2.4. Combining these bounds ultimately leads to,

$$
\begin{aligned}
\sum_{f \in F_{n}}\|f\|_{L^{\infty}\left((0, T) \times \mathbb{R}^{D}\right)} \leq & C\left(\left\|U_{0}\right\|_{L^{\infty}\left(\mathbb{R}^{D}\right)}+\left\|B_{0}\right\|_{L^{\infty}\left(\mathbb{R}^{D}\right)}\right) \\
& +C|\alpha| \sqrt{t}\left(\sum_{f \in F_{n}}\|f\|_{L^{\infty}\left((0, T) \times \mathbb{R}^{D}\right)}\right) \\
& +C \sqrt{T}\left(\sum_{f \in F_{n-1}}\|f\|_{L^{\infty}\left((0, T) \times \mathbb{R}^{D}\right)}\right)^{2} .
\end{aligned}
$$

Let $C_{4}$ heretofore denote twice the value of the constant appearing above. Provided $C_{4}|\alpha| \sqrt{t} \leq 1$ (that is, $|y| \leq \frac{1}{2 C_{4}} \sqrt{t}$ ), we have,

$$
\begin{aligned}
\sum_{f \in F_{n}}\|f\|_{L^{\infty}\left((0, T) \times \mathbb{R}^{D}\right)} \leq & C_{4}\left(\left\|U_{0}\right\|_{L^{\infty}\left(\mathbb{R}^{D}\right)}+\left\|B_{0}\right\|_{L^{\infty}\left(\mathbb{R}^{D}\right)}\right) \\
& +C_{4} \sqrt{T}\left(\sum_{f \in F_{n-1}}\|f\|_{L^{\infty}\left((0, T) \times \mathbb{R}^{D}\right)}\right)^{2} .
\end{aligned}
$$

A consequence of the construction of $F_{0}$ is that,

$$
\sum_{f \in F_{1}}\|f\|_{L^{\infty}\left((0, T) \times \mathbb{R}^{D}\right)} \leq C_{4}\left(\left\|U_{0}\right\|_{L^{\infty}\left(\mathbb{R}^{n}\right)}+\left\|B_{0}\right\|_{L^{\infty}\left(\mathbb{R}^{D}\right)}\right) .
$$

Set,

$$
T_{4}=\frac{1}{16 c_{4}^{4}\left(\left\|U_{0}\right\|_{L^{\infty}\left(\mathbb{R}^{D}\right)}+\left\|B_{0}\right\|_{L^{\infty}\left(\mathbb{R}^{D}\right)}\right)^{2}} .
$$

If $T \leq T_{4}$ we have, proceeding inductively,

$$
\sum_{f \in F_{n}}\|f\|_{L^{\infty}\left(\left(0, T_{4}\right) \times \mathbb{R}^{D}\right)} \leq 2 C_{4}\left(\left\|U_{0}\right\|_{L^{\infty}\left(\mathbb{R}^{D}\right)}+\left\|B_{0}\right\|_{L^{\infty}\left(\mathbb{R}^{D}\right)}\right) .
$$


Setting $\rho(t)=\frac{\sqrt{t}}{2 C_{4}}$ we conclude that for $t \in\left(0, T_{4}\right)$ the sequence of analytic extensions of $U^{(n)}+B^{(n)}$ is uniformly bounded over

$$
D_{t}=\left\{x+i y \in \mathbb{C}^{D}:|y| \leq \rho(t)\right\}
$$

We will eventually desire an improvement of the above bound which leaves $D_{t}$ unchanged. By paying a price on the size of the time interval, we can scale the bound by a factor of $\beta$ for $\beta \in(1 / 2,1]$. The price is to restrict the length of the time interval to be less than $T_{\beta}$ where,

$$
T_{\beta}=\frac{(2 \beta-1)^{2}}{\beta^{4}} T_{4}
$$

Assuming such a restriction, for $n=1$ we have,

$$
\begin{aligned}
\sum_{f \in F_{n}}\|f\|_{L^{\infty}\left((0, T) \times \mathbb{R}^{D}\right)} & \leq C_{4}\left(\left\|U_{0}\right\|_{L^{\infty}\left(\mathbb{R}^{D}\right)}+\left\|B_{0}\right\|_{L^{\infty}\left(\mathbb{R}^{D}\right)}\right) \\
& \leq 2 \beta C_{4}\left(\left\|U_{0}\right\|_{L^{\infty}\left(\mathbb{R}^{D}\right)}+\left\|B_{0}\right\|_{L^{\infty}\left(\mathbb{R}^{D}\right)}\right)
\end{aligned}
$$

and, for subsequent $n$, induction yields,

$$
\sum_{f \in F_{n}}\|f\|_{L^{\infty}\left((0, T) \times \mathbb{R}^{D}\right)} \leq 2 \beta C_{4}\left(\left\|U_{0}\right\|_{L^{\infty}\left(\mathbb{R}^{D}\right)}+\left\|B_{0}\right\|_{L^{\infty}\left(\mathbb{R}^{D}\right)}\right) .
$$

We summarize the preceding discussion in a lemma.

Lemma 3.2.7. Given $U_{0}$ and $B_{0}$ in $L^{\infty}\left(\mathbb{R}^{D}\right)$ there exists a universal constant $C_{4}$ so that, for any $n \in \mathbb{N}$ and $\beta \in(1 / 2,1]$ and for the time,

$$
T_{\beta}=\frac{(2 \beta-1)^{2}}{\beta^{4}} T_{4},
$$

we have,

$$
\begin{aligned}
& \left\|u^{(n)}+i v^{(n)}\right\|_{L^{\infty}(\Omega)}+\left\|b^{(n)}+i c^{(n)}\right\|_{L^{\infty}(\Omega)} \\
& \leq 2 \beta C_{4}\left(\left\|U_{0}\right\|_{L^{\infty}\left(\mathbb{R}^{D}\right)}+\left\|B_{0}\right\|_{L^{\infty}\left(\mathbb{R}^{D}\right)}\right)
\end{aligned}
$$

where,

$$
\Omega=\left\{(x+i y, t) \in \mathbb{C}^{D} \times(0, T):|y| \leq \frac{\sqrt{t}}{2 C_{4}}\right\},
$$

and $0<T \leq T_{\beta}$. 
Convergence of the analytic extensions of the approximations of the mild solutions to the corresponding to analytic extensions of the mild solutions is not attended. It turns out that these limits are also the unique mild solutions to the (spatially) analytic MHD equations, but we forgo a detailed proof of this as it is methodically redundant to existing work (e.g. [62, 64]).

Theorem 3.2.8. Let $U_{0}$ and $B_{0}$ be in $L^{\infty}\left(\mathbb{R}^{D}\right)$ and let $U$ and $B$ be the (unique) real variable solution to $M H D$ on the time interval $\left[0, T^{*}\right)$ where $T^{*}<T_{2}$. Then, for any $0<T<\min \left\{T^{*}, T_{4}\right\}$, and for any $t \in[0, T), U(\cdot, t)$ and $B(\cdot, t)$ have analytic extensions for which the domains of analyticity include $D_{t}$, denote these by $u+i v$ and $b+i c$. Furthermore, for $\beta \in(1 / 2,1]$, there exists $T_{\beta}^{*}=\min \left\{T^{*}, T_{\beta}\right\}$ so that the following bound holds,

$$
\|u+i v\|_{L^{\infty}(\Omega)}+\|b+i c\|_{L^{\infty}(\Omega)} \leq 2 \beta C_{4}\left(\left\|U_{0}\right\|_{L^{\infty}\left(\mathbb{R}^{D}\right)}+\left\|B_{0}\right\|_{L^{\infty}\left(\mathbb{R}^{D}\right)}\right),
$$

where,

$$
\Omega=\left\{(x+i y, t) \in \mathbb{C}^{D} \times\left(0, T_{\beta}^{*}\right):|y| \leq \frac{\sqrt{t}}{2 C_{4}}\right\} .
$$

Proof. Because at each $t$ the approximating functions in the analytic scheme converge on a set containing an accumulation point, namely $\mathbb{R}^{D}$, Vitali's theorem grants that they converge to analytic functions $u+i v$ and $b+i c$. Then, since these agree with $U$ and $B$ on $\mathbb{R}^{D}$, they constitute analytic extensions of $U$ and $B$. The bound follows immediately from the bounds on the approximations.

\subsection{A geometric measure-type regularity criteria for $3 \mathrm{D}$ MHD}

In a recent paper by Z. Grujić (see [61]), a local geometric measure-type condition is shown to prevent finite time singularity formation (with respect to the supremum norm) in NSE with initial data in $L^{\infty}\left(\mathbb{R}^{3}\right)$. The proof utilizes a relatively recent solution (due to Solynin, cf. [99]) to a generalization of the classical Beurling's problem which is concerned with estimating the harmonic measure at the origin and with respect to the unit disk of a closed subset of $[-1,1]$. In the context of 
[61], sharp lower bounds on the uniform radius of spatial analyticity for mild solutions to NSE with initial data in $L^{\infty}\left(\mathbb{R}^{3}\right)$, along with a sparseness condition near the endpoint of a finite length regular time interval $[0, T)$ reduce to a situation aptly estimated via Solynin's result and the harmonic measure maximum principle. This allows one to conclude that $T$ is not a singular time and, thus, the solution extends smoothly past $T$.

In this section we adapt the methods of [61] to the case of $3 \mathrm{D}$ MHD to establish several regularity criteria involving local anisotropic diffusion. Central to these is a sparseness requirement which assumes a bound on the ratio between the (spatial, Lebesgue) measure of the region of intense behavior on some interval centered at the point $x_{0}$ and the length of that interval. More specifically, for a vector field $F$ over $\mathbb{R}^{n}$, we assume for each point $x_{0}$ that there exists a unit vector, $d\left(x_{0}\right)$, and and a magnitude (less than a uniform radius of analyticity), $r\left(x_{0}\right)$, so that

$$
\frac{\left|\Omega_{F} \cap\left(x_{0}-r d, x_{0}+r d\right)\right|}{2 r} \leq \delta,
$$

for some $\delta \in(0,1)$ where $\Omega_{F}$ is the set of points where $|F|$ is above some threshold.

For 3D MHD, sparseness assumptions are natural based on the observationally and numerically motivated theories of turbulence due to the inherent anisotropy evident therein. In particular, in the regime of strong turbulence, the magnetic and velocity fields undergo dynamic alignment and the regions of high spatial complexity - current sheets - are quasi-2D intermittently distributed structures (c.f. [8] and [12]). Thus, it is reasonable to expect that, at least at points where spatial complexity is pronounced, both the velocity field and the magnetic field are sparse in the plane perpendicular direction.

Because the equations for MHD and NSE are formally similar, the mathematical theory of MHD is richly informed by that of NSE (c.f. [96] for the fundamentals). Past work has been done to adapt key regularity results from NSE to MHD. The utilization of coherence introduced in [34] and improved in [5] has been adapted in the case of ideal MHD in [107] and for non-ideal MHD in [65]. Mild solutions for MHD have been studied in the context of $B M O^{-1}$ (c.f. [85]) where the regularity result of Koch-Tataru are extended. Real analyticity of (weak) solutions of 3D MHD has been treated for initial data in $H^{1}$ in [108]; this work 
is also notable as it establishes an analogue of the Beale-Kato-Majda result in the $L^{\infty}$ context (see [109] for an extension to $L^{1}(0, T ; B M O)$ ).

\subsubsection{Requisite material}

Definition 3.3.1. Let $x_{0}$ be a point in $\mathbb{R}^{3}, r>0, S$ an open subset of $\mathbb{R}^{3}$ and $\delta \in(0,1)$. The set $S$ is linearly $\delta$-sparse around $x_{0}$ at scale $r$ if there exists a unit vector $d$ in $S^{2}$ such that,

$$
\frac{\left|S \cap\left(x_{0}-r d, x_{0}+r d\right)\right|}{2 r} \leq \delta
$$

We will be interested in sparseness of super-level sets. For a function $f(x, t)$, a time $t$, and a threshold $M$, a super-level set is defined to be,

$$
\Omega_{f}(t, M)=\left\{x \in \mathbb{R}^{D}:|f(x, t)|>M\right\}
$$

There is a significant amount of freedom in choosing how to relate various parameters and achieve the desired regularity outcome. We begin with a very simple case, Theorem 3.3.3, which most closely mirrors [61]. Here, the sparseness is imposed singly on the intersection of superlevel sets of $U$ and $B$. Consequently, the (local) direction in which $U$ and $B$ are sparse must agree. This is reasonable in the context of the above discussion regarding MHD turbulence. It is, however, formally restrictive and subsequent results are presented to reveal where added subtlety can be achieved. The proof of this result will illustrate Grujić's argument and expedite discussion of later results.

The remaining two theorems achieve the same result as Theorem 3.3.3 but under relaxed assumptions. Theorem 3.3.4 assumes sparseness on each field but with greater independence than in Theorem 3.3.3. A technical parameter specifies a relationship between the thresholds of the superlevel sets for $U$ and $B$ on which these sparseness assumptions are made. Both the direction of sparseness and the scale are, however, independent. Theorem 3.3.5 exploits the linearity of the magnetic field in the induction equation to eliminate this sparseness condition. A cost is here paid by demanding the supremum norm of the magnetic field is suitably bounded (in a fashion dependent on how much we improved the uniform bound) by a scaling of the supremum norm of a single velocity profile. 
The only non-classical result from the theory of harmonic measures which is of interest to us is due to Solynin [99]. It is included for convenience.

Theorem 3.3.2. (Solynin [99]). Let $K$ be a closed subset of $[-1,1]$ such that $|K|=2 \gamma$ for some $0<\gamma<1$. Suppose further that $0 \in \mathbb{D} \backslash K$. Then,

$$
\omega(0, \mathbb{D}, K) \geq \omega\left(0, \mathbb{D}, K_{\gamma}\right)=\frac{2}{\pi} \arcsin \frac{1-(1-\gamma)^{2}}{1+(1-\gamma)^{2}},
$$

where $K_{\gamma}=[-1,-1+\gamma] \cup[1-\gamma, 1]$.

Other necessary results can be found in $[1,89]$ and are also listed in [61].

\subsubsection{Regularity criteria}

Note that heretofore $T_{4}=T_{4}\left(t_{0}\right)$ and $C_{4}=C_{4}\left(t_{0}\right)$ are determined in the context of Theorem 3.2.8 with initial data $U\left(t_{0}\right)$ and $B\left(t_{0}\right)$ for some time $t_{0}$. These mild solutions are, up to a shift in the time variable, just the restrictions of the original solutions to the time interval $\left[t_{0}, t_{0}+T_{4}\right)$. Also, we assume $C_{4} \geq 1$ and observe that, if this is not the case, we can re-determine constants and time interval lengths to reflect the constant $\max \left\{1, C_{4}\right\}$.

Theorem 3.3.3. Suppose $U_{0}, B_{0} \in L^{\infty}$ and consider the corresponding mild solution comprised of $U$ and $B$ defined on an interval of regularity $[0, T)$. Let $\delta \in(0,1), h=h(\delta)=\frac{2}{\pi} \arcsin \frac{1-\delta^{2}}{1+\delta^{2}}, \alpha=\alpha(\delta) \geq \frac{1-h}{h}$ satisfying $\frac{1}{2} \geq \frac{1}{2^{1 / h}\left(2 C_{4}\right)^{\alpha}}$. Assume there exists $\epsilon>0$ so that for any $t_{0} \in(T-\epsilon, T)$, either

1. $t_{0}+T_{4}>T$, or,

2. there exists a time $t=t\left(t_{0}\right) \in\left[t_{0}+T_{4} / 4, t_{0}+T_{4}\right]$ so that, for any $x_{0} \in \mathbb{R}^{3}$, with,

$$
M=\frac{1}{2^{1 / h}\left(2 C_{4}\right)^{\alpha}}\left(\left\|U\left(t_{0}\right)\right\|_{\infty}+\left\|B\left(t_{0}\right)\right\|_{\infty}\right),
$$

and,

$$
S=\Omega_{U}(M, t) \cap \Omega_{B}(M, t),
$$

there exists $r$ with $0<r<\rho\left(T_{4} / 4\right)$ such that $S$ is linearly $\delta$-sparse around $x_{0}$ at scale $r$ in some direction $d$. 
Then, $T$ is not a singular time.

It will be clear from the following proof that (2) needs only hold at finitely many times in $(T-\epsilon, T)$ provided these are suitably spaced.

Proof. In the case of (1) we are done as the solutions with initial data take at $t_{0}$ are uniformly bounded on the interval $\left(t_{0}, t_{0}+T_{4}\right)$ which contains $T$.

In the case of (2) we apply an iterative argument which ultimately reduces to case (1). Our main task is to establish that for any $t_{0}$ there exists a time $t\left(t_{0}\right)$ so that, for all $x_{0} \in \mathbb{R}^{3}$,

$$
\left|U\left(x_{0}, t\right)\right|+\left|B\left(x_{0}, t\right)\right| \leq A,
$$

where $A=\left\|U\left(t_{0}\right)\right\|_{\infty}+\left\|B\left(t_{0}\right)\right\|_{\infty}$. Consequently, the procedure can be repeated with $t$ replacing $t_{0}$ and, as each iteration moves the initial time closer to $T$ by a non-vanishing length, case (1) will eventually be achieved.

To begin, let $t_{0} \in(T-\epsilon, T)$ and $x_{0} \in \mathbb{R}^{3}$ be fixed. Let $t=t\left(t_{0}\right)$ be as in the theorem and, therefore, by the sparseness assumption, there exists a length $r<\rho\left(T_{4} / 4\right)$ and a direction vector $d$ so that,

$$
\frac{S \cap\left(x_{0}-r d, x_{0}+r d\right) \mid}{2 r} \leq \delta .
$$

Observing the the MHD system is rotationally and translationally invariant, let $Q$ denote the transformation (rotation and translation) taking $x_{0}$ to 0 and directing $d$ to be parallel to the first coordinate vector, $e_{1}$. Let $U_{x_{0}, Q}$ and $B_{x_{0}, Q}$ comprise a mild solution to the transformed MHD with initial data taken at $t_{0}$.

By Theorem 3.2.8, on $\left(0, T_{4}\right), U_{x_{0}, Q}$ and $B_{x_{0}, Q}$ have analytic extensions satisfying the uniform bound,

$$
|u+i v|+|b+i c| \leq 2 C_{4} A,
$$

where the bounded terms are the appropriate analytic extensions. Focussing on the extension of the first spatial coordinate axis, hereafter called just the real axis, we see the domains of analyticity contain the disk centred at 0 of radius $r, D_{r}$ because $r<\rho\left(T_{4} / 4\right) \leq \rho(t)$. 
Let $K$ be the complement in $[-r, r]$ of $Q$ applied to $S \cap\left(x_{0}-r d, x_{0}+\right.$ $r d)$. If $0 \in K$ then,

$$
\left|U_{x_{0}, Q}(0, t)\right| \leq M \leq \frac{1}{2} A,
$$

and, as the same bound holds on $\left|B_{x_{0}, Q}(0, t)\right|$, we are done. If $0 \notin K$ we turn to the theory of harmonic measures.

To apply the harmonic measure maximum principle (c.f. [1] pg. 39) observe that, uniformly in $D_{r}$ we have,

$$
|u+i v| \leq 2 C_{4} A,
$$

while uniformly in $K$ we have,

$$
|u+i v| \leq M
$$

and, consequently,

$$
\left|U_{x_{0}, Q}(0, t)\right| \leq\left(\frac{A}{2^{1 / h}\left(2 C_{4}\right)^{\alpha}}\right)^{\omega\left(0, D_{r}, K\right)}\left(2 C_{4} A\right)^{1-\omega\left(0, D_{r}, K\right)} .
$$

The sparseness assumption entails that $|K| \geq 2 r(1-\delta)$. Letting $\frac{1}{r} K=\{z \in \mathbb{C}: r z \in K\}$, we obtain $\left|\frac{1}{r} K\right| \geq 2(1-\delta)$. Applying Theorem 3.3.2 with $\gamma=1-\delta$ to a subset $K^{\prime} \subset K$ where $\left|K^{\prime}\right|=2(1-\delta)$ yields (noting that harmonic measure increases with $K$ ),

$\omega\left(0, D_{1}, \frac{1}{r} K\right) \geq \omega\left(0, D_{1}, \frac{1}{r} K^{\prime}\right) \geq \omega\left(0, D_{1}, K_{\gamma}\right)=\frac{2}{\pi} \arcsin \frac{1-(\delta)^{2}}{1+(\delta)^{2}}=h$.

Since harmonic measure is invariant under conformal mappings, it is invariant under the mapping $z \mapsto r z$. The previous inequality then implies that,

$$
\omega\left(0, D_{r}, K\right) \geq h .
$$

Combining our bounds (noting $C_{4}>1$ and $M \leq 2 C_{4} A$ ) we have,

$$
\left|U_{x_{0}, Q}(0, t)\right| \leq\left(\frac{A}{2^{1 / h}\left(2 C_{4}\right)^{\alpha}}\right)^{h}\left(2 C_{4} A\right)^{1-h} \leq \frac{1}{2} A .
$$

And, undoing our transformation $Q$,

$$
\left|U\left(x_{0}, t\right)\right| \leq \frac{1}{2} A .
$$


Proceeding identically results in the same bound for $\left|B\left(x_{0}, t\right)\right|$ which gives the conclusion for $x_{0}$, namely,

$$
\left|U\left(x_{0}, t\right)\right|+\left|B\left(x_{0}, t\right)\right| \leq\left\|U\left(t_{0}\right)\right\|_{\infty}+\left\|B\left(t_{0}\right)\right\|_{\infty} .
$$

As our selection of $x_{0}$ was arbitrary this holds uniformly and the iterative argument outlined at the onset of the proof allows us to conclude that $T$ is not a singular time.

Theorem 3.3.4. Suppose $U_{0}, B_{0} \in L^{\infty}$ and consider the corresponding mild solution comprised of $U$ and $B$ defined on an interval of regularity $[0, T)$. Let $\delta \in(0,1), h=h(\delta)=\frac{2}{\pi} \arcsin \frac{1-\delta^{2}}{1+\delta^{2}}, \alpha=\alpha(\delta) \geq \frac{1-h}{h}$, and $\gamma \in(0,1)$.

Assume there exists $\epsilon>0$ so that for any $t_{0} \in(T-\epsilon, T)$, either

1. $t_{0}+T_{4}>T$, or,

2. there exists a time $t=t\left(t_{0}\right) \in\left[t_{0}+T_{4} / 4, t_{0}+T_{4}\right]$ so that, for any $x_{0} \in \mathbb{R}^{3}$, there exist $r_{U}$ and $r_{B}$ so that following sparseness conditions are met:

- $\Omega_{U}\left(t, M_{U}\right)$ is linearly $\delta$-sparse around $x_{0}$ at scale $r_{U}$ where $0<r_{U} \leq \rho\left(T_{4} / 4\right)$ and $M_{U}=\frac{\gamma}{\left(2 C_{4}\right)^{\alpha}}\left(\left\|U\left(t_{0}\right)\right\|_{\infty}+\left\|B\left(t_{0}\right)\right\|_{\infty}\right)$, and,

- $\Omega_{B}\left(t, M_{B}\right)$ is linearly $\delta$-sparse around $x_{0}$ at scale $r_{B}$ where $0<r_{B} \leq \rho\left(T_{4} / 4\right)$ and $M_{B}=\frac{\left(1-\gamma^{h}\right)^{1 / h}}{\left(2 C_{4}\right)^{\alpha}}\left(\left\|U\left(t_{0}\right)\right\|_{\infty}+\left\|B\left(t_{0}\right)\right\|_{\infty}\right)$.

Then, $T$ is not a singular time.

Proof. The same iterative argument seen in the proof of Theorem 3.3.3 can be applied once we obtain for all $x_{0}$ the bounds,

$$
\left|U\left(x_{0}, t\right)\right| \leq \gamma^{h}\left(\left\|U\left(t_{0}\right)\right\|_{\infty}+\left\|B\left(t_{0}\right)\right\|_{\infty}\right),
$$

and,

$$
\left|B\left(x_{0}, t\right)\right| \leq\left(1-\gamma^{h}\right)\left(\left\|U\left(t_{0}\right)\right\|_{\infty}+\left\|B\left(t_{0}\right)\right\|_{\infty}\right) .
$$

This is shown by cases depending on the inclusion of $x_{0}$ in the relevant super-level sets. In the case where $x_{0} \in \Omega_{U}\left(t, M_{U}\right)$, an identical 
argument to that in the previous proof leads us to the estimate,

$$
\begin{aligned}
\left|U_{x_{0}, Q}(0, t)\right| \leq & \left.\frac{\gamma}{\left(2 C_{4}\right)^{\alpha}}\left(\left\|U\left(t_{0}\right)\right\|_{\infty}+\left\|B\left(t_{0}\right)\right\|_{\infty}\right)\right)^{\omega\left(0, D_{r_{U}}, K\right)} \\
& \cdot\left(2 C_{4}\left(\left\|U\left(t_{0}\right)\right\|_{\infty}+\left\|B\left(t_{0}\right)\right\|_{\infty}\right)\right)^{1-\omega\left(0, D_{r_{U}}, K\right)} .
\end{aligned}
$$

Since $1 \geq \omega\left(0, D_{r_{U}}, K_{U}\right)>h>0$ and $\gamma \in(0,1), \gamma^{h} \geq \gamma^{\omega\left(0, D_{r_{U}}, K_{U}\right)}$. The desired bound follows. The case for $x_{0} \in \Omega_{B}\left(t, M_{B}\right)$ is identical up to labeling.

If $x_{0} \notin \Omega_{U}\left(t, M_{U}\right)$ then we have,

$$
\left|U\left(x_{0}, t\right)\right| \leq \frac{\gamma}{\left(2 C_{4}\right)^{\alpha}}\left(\left\|U\left(t_{0}\right)\right\|_{\infty}+\left\|B\left(t_{0}\right)\right\|_{\infty}\right) .
$$

Since $h, \gamma \in(0,1), \alpha>0$, and $2 C_{4}>1$, we clearly see that,

$$
\frac{\gamma}{\left(2 C_{4}\right)^{\alpha}} \leq \gamma^{h}
$$

which leads to the desired bound.

If $x_{0} \notin \Omega_{B}\left(t, M_{B}\right)$ then we similarly have,

$$
\left|B\left(x_{0}, t\right)\right| \leq \frac{\left(1-\gamma^{h}\right)^{1 / h}}{\left(2 C_{4}\right)^{\alpha}}\left(\left\|U\left(t_{0}\right)\right\|_{\infty}+\left\|B\left(t_{0}\right)\right\|_{\infty}\right),
$$

and, observing that,

$$
\frac{\left(1-\gamma^{h}\right)^{1 / h}}{\left(2 C_{4}\right)^{\alpha}} \leq\left(1-\gamma^{h}\right)
$$

the desired estimate follows.

As we have dealt with each case we conclude that the initially stated bounds are valid and, iterating, we see that $T$ is not a singular time.

Theorem 3.3.5. Suppose $U_{0}, B_{0} \in L^{\infty}$ and consider the corresponding mild solution comprised of $U$ and $B$ defined on an interval of regularity $[0, T)$. Let $\delta \in(0,1), h=h(\delta)=\frac{2}{\pi} \arcsin \frac{1-\delta^{2}}{1+\delta^{2}}, \alpha=\alpha(\delta) \geq \frac{1-h}{h}$. Additionally, let $\beta \in(1 / 2,1)$ satisfy $\beta^{1-h} \geq 1 /\left(2 C_{4}\right)^{\alpha}$.

Assume there exists $\epsilon>0$ and a collection of times $t_{0}, t_{1}, \ldots, t_{k} \in$ $(T-\epsilon, T)$, so that 
1. $t_{k}+T_{4}>T$,

2. $t_{i+1} \in\left[t_{i}+T_{\beta}\left(t_{i}\right) / 4, t_{i}+T_{\beta}\left(t_{i}\right)\right]$, and,

3. the following two criteria are met:

- for each $i$ and for any $x_{0}$ there exists $r$ so that $\Omega_{U}\left(t_{i+1}, M\right)$ is linearly $\delta$-sparse around $x_{0}$ at scale $r$ where $0<r \leq \rho\left(T_{\beta} / 4\right)$ and $M=\frac{1}{\left(2 C_{4}\right)^{\alpha}}\left(\left\|U\left(t_{i}\right)\right\|_{\infty}+\left\|B\left(t_{i}\right)\right\|_{\infty}\right)$, and,

- $2 C_{3}\left\|B\left(t_{0}\right)\right\|_{\infty} \leq\left(1-\beta^{1-h}\right)\left(\left\|U\left(t_{0}\right)\right\|_{\infty}+\left\|B\left(t_{0}\right)\right\|_{\infty}\right)$.

Then, $T$ is not a singular time.

In the above we specified a particular time at which to begin our iterative argument in order to not trivialize the condition on the magnetic field. Note that in the context of the above theorem $\beta$ and $T_{\beta}$ reference Theorem 3.2.8. Also, when $C_{4} \geq 1$ we have $\beta^{1-h} \geq 1 /\left(2 C_{4}\right)^{\alpha}$.

Proof. We obtain for all $x_{0}$ the bounds,

$$
\left|U\left(x_{0}, t_{1}\right)\right| \leq \beta^{h}\left(\left\|U\left(t_{0}\right)\right\|_{\infty}+\left\|B\left(t_{0}\right)\right\|_{\infty}\right),
$$

and,

$$
\left|B\left(x_{0}, t_{1}\right)\right| \leq\left(1-\beta^{h}\right)\left(\left\|U\left(t_{0}\right)\right\|_{\infty}+\left\|B\left(t_{0}\right)\right\|_{\infty}\right) .
$$

Applying the now familiar argument with the modification that the disk on which we are taking $U_{x_{0}, t_{1}}$ to be analytic is that on which the bound $\left|U_{x_{0}, t_{1}}\right| \leq 2 \beta\left(\left\|U\left(t_{0}\right)\right\|_{\infty}+\left\|B\left(t_{0}\right)\right\|_{\infty}\right)$ holds (see Lemma 3.2.7), yields the desired bound on $U$, namely,

$$
\left|U\left(x_{0}, t_{1}\right)\right| \leq \beta^{1-h}\left(\left\|U\left(t_{0}\right)\right\|_{\infty}+\left\|B\left(t_{0}\right)\right\|_{\infty}\right) .
$$

A complementary estimate (noting $\left.T_{3} \geq T_{\beta}\right)$ on $\left|B\left(x_{0}, t_{1}\right)\right|$ follows from the second assumption and Corollary 3.2.6. These grant that,

$$
\left|B\left(x_{0}, t_{1}\right)\right| \leq 2 C_{3}\left\|B\left(t_{0}\right)\right\|_{\infty} \leq\left(1-\beta^{1-h}\right)\left(\left\|B\left(t_{0}\right)\right\|_{\infty}+\left\|U\left(t_{0}\right)\right\|_{\infty}\right) .
$$

Combining these bounds leads to,

$$
\left|U\left(x_{0}, t_{1}\right)\right|+\left|B\left(x_{0}, t_{1}\right)\right| \leq\left\|U\left(t_{0}\right)\right\|_{\infty}+\left\|B\left(t_{0}\right)\right\|_{\infty} .
$$

Establishing identical relationships for $t_{i+1}$ and $t_{i}$ follows in the exact same manner and the iterative argument then grants that $T$ is not a singular time. 


\subsection{Local estimates for the analyticity ra- dius of solutions of the Navier-Stokes equations with locally analytic forcing}

We return to the subject of analyticity radii and are interested in whether or not local lower bounds can be determined for flows subjected to real-analytic forcing which have a possibly non-uniform analyticity radius. To provide context, let $f$ denote the forcing term from $3 \mathrm{D}$ NSE, $\lambda_{f}(x, t)$ the radius of spatial analyticity of $f$ at $(x, t)$, and let,

$$
\lambda_{f, T}(x)=\inf _{t \in(0, T]} \lambda_{f}(x, t) .
$$

Fix a point $x_{*} \in \mathbb{R}^{3}$ and specify the length $r_{*}=\lambda_{f, T}\left(x_{*}\right) / 2$. By $B_{*}$ denote the ball of radius $r_{*}$ centered at $x_{*}$. Then, if $x \in 2 B_{*}$ it follows that $\lambda_{f, T}(x) \geq 2 r_{*}-\left|x-x_{*}\right|$, and, consequently, $f(x, t)$ is the restriction to $\mathbb{R}^{3}$ of a function $F(x, y, t)+i G(x, y, t)$ which is defined and spatially analytic on the domain,

$$
\Omega_{f, T}\left(x_{*}\right)=\left\{x+i y \in \mathbb{C}^{3}: x \in 2 B_{*},|y|<2 r_{*}-\left|x-x_{*}\right|\right\},
$$

provided $t \in(0, T]$.

A non-negative test function $\psi$ can be constructed which is supported on $2 B_{*}$, evaluates to 1 on $B_{*}$, is radially non-increasing in $\left|x-x_{*}\right|$, and additionally satisfies the estimates,

$$
|\psi(x)| \leq \frac{2 r_{*}-\left|x-x_{*}\right|}{r_{*}}
$$

and,

$$
\|\nabla \psi\|_{\infty} \leq \frac{C}{r^{*}}
$$

Let $\alpha \in \mathbb{R}^{3}$ satisfy $(x, \alpha \psi(x) t, t)$ is in the domain of analyticity of $F+i G$ provided $t \leq r_{*}^{2}$; denote the set of all permissible vectors $\alpha$ by $S_{f}$. Let $F_{\alpha}(x, t)=F(x, \alpha \psi(x) t, t)$ and $G_{\alpha}(x, t)=G(x, \alpha \psi(x) t, t)$.

Theorem 3.4.1. Assume that $u_{0}$ is a divergence free vector field in the Sobolev space $W^{1, q}\left(\mathbb{R}^{3}\right)$ for some $q>3$ and that $f$ agrees with the 
restriction of the analytic function $F+i G$ to $2 B_{*}$. If for some positive time $T$ we have,

$$
M_{f}(T):=\sup _{\alpha \in S_{f}}\left(\sup _{0<\tau<T}\left\|F_{\alpha}(\tau)\right\|_{L^{q}\left(\mathbb{R}^{3}\right)}+\sup _{0<\tau<T}\left\|G_{\alpha}(\tau)\right\|_{L^{q}\left(\mathbb{R}^{3}\right)}\right)<\infty,
$$

and, for some $r>2 q /(q-3)$,

$$
M_{f}^{\prime}(T):=\sup _{\alpha \in S_{f}}\left[\left(\int_{0}^{T}\left\|\nabla F_{\alpha}(\tau)\right\|_{L^{q}}^{r} d t\right)^{\frac{1}{r}}+\left(\int_{0}^{T}\left\|\nabla G_{\alpha}(\tau)\right\|_{L^{q}}^{r} d t\right)^{\frac{1}{r}}\right],
$$

is finite, then there exists a positive time $T_{1}$ occurring prior to $T$ so that $3 D$ NSE has a solution $u \in C\left(\left[0, T_{1}\right), L^{q}\left(\mathbb{R}^{3}\right)\right)$ which, at any time $t \in\left(0, T_{1}\right)$, agrees with the restriction to $B_{*}$ of a function $u(x, y, t)+$ $i v(x, y, t)$ which is analytic in the region,

$$
\Omega_{*}(t)=\left\{x+i y \in \mathbb{C}^{3}: x \in B_{*},|y|<\frac{\sqrt{t}}{4 C_{0}}\right\},
$$

for a universal constant $C_{0}$.

Remark 3.4.1. The time $T_{1}$ will be obtained in terms of quantities involving $\left\|u_{0}\right\|_{W^{1, q}}$ as well as $M_{f}\left(T_{1}\right)$ and $M_{f}^{\prime}\left(T_{1}\right)$ (see in particular (3.4.20) and (3.4.30)). The proof, which is contained in Section 3.4.3, additionally establishes that,

$$
\sup _{0<t<T_{1}}\left\|U_{\alpha}(t)\right\|_{L^{q}}+\sup _{0<t<T_{1}}\left\|V_{\alpha}(t)\right\|_{L^{q}} \leq C\left\|u_{0}\right\|_{q}
$$

and,

$$
t^{\frac{r-2}{2 r}}\left[\left(\int_{0}^{t}\left\|\nabla U_{\alpha}(\tau)\right\|_{L^{q}}^{r} d \tau\right)^{\frac{1}{r}}+\left(\int_{0}^{t}\left\|\nabla V_{\alpha}(\tau)\right\|_{L^{q}}^{r} d \tau\right)^{\frac{1}{r}}\right] \leq C\left\|u_{0}\right\|_{q},
$$

for $0<t<T_{1}$. The exponent $r$ is introduced for technical reasons.

We prove Theorem 3.4.1 by studying complex perturbations of elements of a classical approximation scheme for 3D NSE. The scheme is constructed by setting $u^{(0)}=p^{(0)}=0$ and, for $n \geq 1$, defining $u^{(n)}$ by successively solving the system,

$$
\begin{cases}\partial_{t} u^{(n)}-\Delta u^{(n)}=-u^{(n-1)} \cdot \nabla u^{(n-1)}-\nabla p^{(n-1)}+f & \text { in } \mathbb{R}^{3} \times(0, T) \\ \nabla \cdot u^{(n)}=0 & \text { in } \mathbb{R}^{3} \times(0, T) \\ \Delta p^{(n-1)}=-\partial_{i} \partial_{j}\left(u_{i}^{(n-1)} u_{j}^{(n-1)}\right) & \text { in } \mathbb{R}^{3} \times(0, T) \\ u^{(n)}(\cdot, 0)=u_{0}(\cdot) & \text { in } \mathbb{R}^{3} .\end{cases}
$$


This scheme has been studied extensively (cf. [72]) and its convergence to a solution of 3D NSE in $C\left([0, T) ; L^{q}\left(\mathbb{R}^{3}\right)\right)$ is known for appropriately small values of $T$.

Let $\alpha \in S_{f}$ so that $(x, \alpha \psi(x) t, t)$ is in the domain of analyticity of $F+i G$ provided $t \leq r_{*}^{2}$. The smoothing properties of the heat equation and the analyticity of $f$ imply that $u^{(n)}$ and $p^{(n)}$ are themselves restrictions to $\mathbb{R}^{3}$ of functions $U^{(n)}+i V^{(n)}$ and $P^{(n)}+i \Pi^{(n)}$ which are defined (at least) on $\mathbb{R}^{3} \cup \Omega_{f, T}\left(x_{*}\right)$, are analytic on $\Omega_{f, T}\left(x_{*}\right)$ and, at points in $\Omega_{f, T}\left(x_{*}\right) \times(0, T)$, satisfy,

$$
\begin{aligned}
& \partial_{t} U^{(n)}-\Delta U^{(n)}=-U^{(n-1)} \cdot \nabla U^{(n-1)}+V^{(n-1)} \cdot \nabla V^{(n-1)}-\nabla P^{(n-1)}+F \\
& \partial_{t} V^{(n)}-\Delta V^{(n)}=-U^{(n-1)} \cdot \nabla V^{(n-1)}-V^{(n-1)} \cdot \nabla U^{(n-1)}-\nabla \Pi^{(n-1)}+G \\
& \Delta P^{(n-1)}=-\partial_{i} \partial_{j}\left(U_{i}^{(n-1)} U_{j}^{(n-1)}-V_{i}^{(n-1)} V_{j}^{(n-1)}\right) \\
& \Delta \Pi^{(n-1)}=-2 \partial_{i} \partial_{j}\left(U_{i}^{(n-1)} V_{j}^{(n-1)}\right) \\
& \nabla \cdot U^{(n)}=0 \text { and } \nabla \cdot V^{(n)}=0
\end{aligned}
$$

Note that $(x, \alpha t \psi(x), t)$ is in the domain of analyticity of $U^{(n)}+i V^{(n)}$ and $P^{(n)}+i \Pi^{(n)}$. Let $U_{\alpha}^{(n)}(x, t)=U^{(n)}(x, \alpha t \psi(x), t)$ with analogous definitions holding for $V_{\alpha}^{(n)}, P_{\alpha}^{(n)}$, and $\Pi_{\alpha}^{(n)}$ and the forcing terms. We presently derive evolution equations for $U_{\alpha}^{(n)}$ and $V_{\alpha}^{(n)}$ and kinematic equations for $P_{\alpha}^{(n)}$ and $\Pi_{\alpha}^{(n)}$.

Since the Cauchy-Riemann system,

$$
\begin{cases}\partial_{i} U_{\alpha}^{(n)}=\partial_{i}^{*} V_{\alpha}^{(n)}, & \partial_{i}^{*} U_{\alpha}^{(n)}=-\partial_{i} V_{\alpha}^{(n)}, \\ \partial_{i} P_{\alpha}^{(n)}=\partial_{i}^{*} \Pi_{\alpha}^{(n)}, & \partial_{i}^{*} P_{\alpha}^{(n)}=-\partial_{i} \Pi_{\alpha}^{(n)},\end{cases}
$$

is satisfied on $\Omega_{f, T}\left(x_{*}\right)$ (note that $\partial_{i}^{*}$ denotes the partial derivative in the $i$-th complex variable), we see that time derivatives of $U_{\alpha}^{(n)}$ and $V_{\alpha}^{(n)}$ satisfy,

$$
\begin{aligned}
& \partial_{t} U_{\alpha}^{(n)}(x, t)=\left(\partial_{t} U^{(n)}\right)(x, \alpha t \psi(x), t)-\alpha_{l} \psi(x)\left(\partial_{l} V^{(n)}\right)(x, \alpha t \psi(x), t) \\
& \partial_{t} V_{\alpha}^{(n)}(x, t)=\left(\partial_{t} V^{(n)}\right)(x, \alpha t \psi(x), t)+\alpha_{l} \psi(x)\left(\partial_{l} U^{(n)}\right)(x, \alpha t \psi(x), t),
\end{aligned}
$$

and that spatial derivatives satisfy,

$$
\begin{aligned}
& \partial_{j} U_{\alpha}^{(n)}(x, t)=\partial_{j} U^{(n)}(x, \alpha t \psi(x), t)-\alpha_{l} t \partial_{j} \psi(x)\left(\partial_{l} V^{(n)}\right)(x, \alpha t \psi(x), t) \\
& \partial_{j} V_{\alpha}^{(n)}(x, t)=\partial_{j} V^{(n)}(x, \alpha t \psi(x), t)+\alpha_{l} t \partial_{j} \psi(x)\left(\partial_{l} U^{(n)}\right)(x, \alpha t \psi(x), t) .
\end{aligned}
$$


Provided,

$$
|\alpha| t \leq \frac{r_{*}}{C_{0}}
$$

where $C_{0}$ is a sufficiently large positive constant the value of which will be further refined as the proof develops, we obtain algebraically that,

$$
\begin{aligned}
& \left(\partial_{j} U^{(n)}\right)(x, \alpha t \psi(x), t)=b_{j k}^{11} \partial_{k} U_{\alpha}^{(n)}+b_{j k}^{12} \partial_{k} V_{\alpha}^{(n)} \\
& \left(\partial_{j} V^{(n)}\right)(x, \alpha t \psi(x), t)=b_{j k}^{21} \partial_{k} U_{\alpha}^{(n)}+b_{j k}^{22} \partial_{k} V_{\alpha}^{(n)},
\end{aligned}
$$

where the functions $b_{j k}^{l m}$ are smooth, non-negative, bounded, and have gradients supported in $2 B_{*}$. Extending this reasoning to second derivatives yields,

$$
\begin{aligned}
\left(\partial_{i} \partial_{j} U^{(n)}\right)(x, \alpha t \psi(x), t)= & b_{k l}^{11} \partial_{l}\left(b_{j m}^{11} \partial_{m} U_{\alpha}^{(n)}(x, t)+b_{j m}^{12} \partial_{m} V_{\alpha}^{(n)}(x, t)\right) \\
& +b_{k l}^{12} \partial_{l}\left(b_{j m}^{21} \partial_{m} U_{\alpha}^{(n)}(x, t)+b_{j m}^{22} \partial_{m} V_{\alpha}^{(n)}(x, t)\right) \\
\left(\partial_{i} \partial_{j} V^{(n)}\right)(x, \alpha t \psi(x), t)= & b_{k l}^{21} \partial_{l}\left(b_{j m}^{11} \partial_{m} U_{\alpha}^{(n)}(x, t)+b_{j m}^{12} \partial_{m} V_{\alpha}^{(n)}(x, t)\right) \\
& +b_{k l}^{22} \partial_{l}\left(b_{j m}^{21} \partial_{m} U_{\alpha}^{(n)}(x, t)+b_{j m}^{22} \partial_{m} V_{\alpha}^{(n)}(x, t)\right) .
\end{aligned}
$$

Again using the Cauchy-Riemann system as well as repeated applications of the chain rule, we obtain a useful formula for the Laplacians of $U^{(n)}$ and $V^{(n)}$,

$$
\begin{aligned}
\left(\Delta U^{(n)}\right)(x, \alpha t \psi(x), t)= & \Delta U_{\alpha}^{(n)}(x, t) \\
& +\partial_{l}\left(a_{l k}^{11} \partial_{k} U_{\alpha}^{(n)}(x, t)\right)+\partial_{l}\left(a_{l k}^{12} \partial_{k} V_{\alpha}^{(n)}(x, t)\right) \\
& +\tilde{c}_{l}^{11} \partial_{l} U_{\alpha}^{(n)}(x, t)+\tilde{c}_{l}^{12} \partial_{l} V_{\alpha}^{(n)}(x, t), \\
\left(\Delta V^{(n)}\right)(x, \alpha t \psi(x), t)= & \Delta V_{\alpha}^{(n)}(x, t) \\
& +\partial_{l}\left(a_{l k}^{21} \partial_{k} U_{\alpha}^{(n)}(x, t)\right)+\partial_{l}\left(a_{l k}^{22} \partial_{k} V_{\alpha}^{(n)}(x, t)\right), \\
& +\tilde{c}_{l}^{21} \partial_{l} U_{\alpha}^{(n)}(x, t)+\tilde{c}_{l}^{22} \partial_{l} V_{\alpha}^{(n)}(x, t),
\end{aligned}
$$

where the coefficient functions are supported on $2 B_{*}$ and satisfy,

$$
\left\|a_{j k}^{l m}\right\|_{\infty} \lesssim|\alpha| t|\nabla \psi|
$$


and,

$$
\left\|\tilde{c}_{j}^{l m}\right\|_{\infty} \lesssim\left\|\nabla a_{j k}^{l m}\right\|_{\infty} \lesssim \frac{|\alpha| t|\nabla \psi|}{r_{*}}
$$

The smallness condition (3.4.1) thus ensures that,

$$
\left\|a_{j k}^{l m}\right\|_{\infty} \leq \frac{C}{C_{0}}
$$

and,

$$
\left\|\tilde{c}_{j}^{l m}\right\|_{\infty} \leq \frac{C}{r_{*} C_{0}}
$$

The representations involving spatial derivatives hold for any conjugate pair analytic on $\Omega_{f, T}\left(x_{*}\right)$; in particular, they hold for $P^{(n)}, P_{\alpha}^{(n)}$, $\Pi^{(n)}$, and $\Pi_{\alpha}^{(n)}$. They also lead to evolution equations for $U_{\alpha}^{(n)}$ and $V_{\alpha}^{(n)}$, namely,

$$
\begin{aligned}
\partial_{t} & U_{\alpha}^{(n)}-\Delta U_{\alpha}^{(n)} \\
= & -U_{\alpha, l}^{(n-1)}\left[b_{l k}^{11} \partial_{k} U_{\alpha}^{(n-1)}+b_{l k}^{12} \partial_{k} V_{\alpha}^{(n-1)}\right] \\
& +V_{\alpha, l}^{(n-1)}\left[b_{l k}^{21} \partial_{k} U_{\alpha}^{(n-1)}+b_{l k}^{22} \partial_{k} V_{\alpha}^{(n-1)}\right] \\
& -b_{l k}^{11} \partial_{k} P_{\alpha}^{(n-1)}-b_{l k}^{12} \partial_{k} \Pi_{\alpha}^{(n-1)}+\alpha_{l} c_{l m}^{11} \partial_{m} U_{\alpha}^{(n)}+\alpha_{l} c_{l m}^{12} \partial_{m} V_{\alpha}^{(n)} \\
& +\partial_{l}\left(a_{l k}^{11} \partial_{k} U_{\alpha}^{(n)}\right)+\partial_{l}\left(a_{l k}^{12} \partial_{k} V_{\alpha}^{(n)}\right)+\tilde{c}_{l}^{11} \partial_{l} U_{\alpha}^{(n)}+\tilde{c}_{l}^{12} \partial_{l} V_{\alpha}^{(n)}+F_{\alpha},
\end{aligned}
$$

and,

$$
\begin{aligned}
\partial_{t} & V_{\alpha}^{(n)}-\Delta V_{\alpha}^{(n)} \\
= & -V_{\alpha, l}^{(n-1)}\left[b_{l k}^{11} \partial_{k} U_{\alpha}^{(n-1)}+b_{l k}^{12} \partial_{k} V_{\alpha}^{(n-1)}\right] \\
& -U_{\alpha, l}^{(n-1)}\left[b_{l k}^{21} \partial_{k} U_{\alpha}^{(n-1)}+b_{l k}^{22} \partial_{k} V_{\alpha}^{(n-1)}\right] \\
& -b_{l k}^{21} \partial_{k} P_{\alpha}^{(n-1)}-b_{l k}^{22} \partial_{k} \Pi_{\alpha}^{(n-1)}+\alpha_{l} c_{l m}^{21} \partial_{m} U_{\alpha}^{(n)}+\alpha_{l} c_{l m}^{22} \partial_{m} V_{\alpha}^{(n)} \\
& +\partial_{l}\left(a_{l k}^{21} \partial_{k} U_{\alpha}^{(n)}\right)+\partial_{l}\left(a_{l k}^{22} \partial_{k} V_{\alpha}^{(n)}\right)+\tilde{c}_{l}^{21} \partial_{l} U_{\alpha}^{(n)}+\tilde{c}_{l}^{22} \partial_{l} V_{\alpha}^{(n)}+G_{\alpha},
\end{aligned}
$$

where $c_{l m}^{i j}$ are smooth, bounded, and supported in $2 B_{*}$, and kinematic 
equations for $P_{\alpha}^{(n)}$ and $\Pi_{\alpha}^{(n)}$, namely,

$$
\begin{aligned}
-\Delta P_{\alpha}^{(n)}= & \partial_{l}\left(a_{l k}^{11} \partial_{k} P_{\alpha}^{(n)}\right)+\partial_{l}\left(a_{l k}^{12} \partial_{k} \Pi_{\alpha}^{(n)}\right)+\tilde{c}_{l}^{11} \partial_{l} P_{\alpha}^{(n)}+\tilde{c}_{l}^{12} \partial_{l} \Pi_{\alpha}^{(n)} \\
& +b_{i k}^{11} \partial_{k}\left(b_{j l}^{11} \partial_{l}\left(U_{\alpha, i}^{(n)} U_{\alpha, j}^{(n)}-V_{\alpha, i}^{(n)} V_{\alpha, j}^{(n)}\right)+b_{j l}^{12} \partial_{l}\left(2 U_{\alpha, i}^{(n)} V_{\alpha, j}^{(n)}\right)\right) \\
& +b_{i k}^{12} \partial_{k}\left(b_{j l}^{21} \partial_{l}\left(U_{\alpha, i}^{(n)} U_{\alpha, j}^{(n)}-V_{\alpha, i}^{(n)} V_{\alpha, j}^{(n)}\right)+b_{j l}^{22} \partial_{l}\left(2 U_{\alpha, i}^{(n)} V_{\alpha, j}^{(n)}\right)\right)
\end{aligned}
$$

and,

$$
\begin{aligned}
-\Delta \Pi_{\alpha}^{(n)}= & \left.\partial_{l}\left(a_{l k}^{21} \partial_{k} P_{\alpha}^{(n)}\right)+\partial_{l}\left(a_{l k}^{22} \partial_{k} \Pi_{\alpha}^{(n)}\right)+\tilde{c}_{l}^{21} \partial_{l} P_{\alpha}^{(n)}\right)+\tilde{c}_{l}^{22} \partial_{l} \Pi_{\alpha}^{(n)} \\
& +b_{i k}^{21} \partial_{k}\left(b_{j l}^{11} \partial_{l}\left(U_{\alpha, i}^{(n)} U_{\alpha, j}^{(n)}-V_{\alpha, i}^{(n)} V_{\alpha, j}^{(n)}\right)+b_{j l}^{12} \partial_{l}\left(2 U_{\alpha, i}^{(n)} V_{\alpha, j}^{(n)}\right)\right) \\
& +b_{i k}^{12} \partial_{k}\left(b_{j l}^{21} \partial_{l}\left(U_{\alpha, i}^{(n)} U_{\alpha, j}^{(n)}-V_{\alpha, i}^{(n)} V_{\alpha, j}^{(n)}\right)+b_{j l}^{12} \partial_{l}\left(2 U_{\alpha, i}^{(n)} V_{\alpha, j}^{(n)}\right)\right)
\end{aligned}
$$

In the remainder of this section we carry out an inductive argument which is motivated by the mild solution approach taken in [72]. Several modifications are needed to accommodate the various terms appearing in (3.4.4)-(3.4.7). The first is our handling of the pressure; this is done directly using the Calderon-Zygmund theory in place of involving the Leray projector and is addressed in subsection 3.4.1. The systems (3.4.4) and (3.4.5) include linear second order terms as well as nonlinear terms with no structural cancellation due to incompressibility. To accommodate these the $L^{r}\left(0, T ; L^{q}\left(\mathbb{R}^{3}\right)\right)$ norms of spatial gradients need to be considered where $r$ is a value greater but close to 2 ; this contrasts Kato's work wherein controlling the $L^{\infty}\left(0, T ; L^{q}\left(\mathbb{R}^{3}\right)\right)$ norms sufficed. These estimates are carried out in subsection 3.4.2 and constitute the bulk of the work required to prove Theorem 3.4.1. The proof itself is contained in subsection 3.4.3.

\subsubsection{Estimates in $L^{s}$ for $P_{\alpha}^{(n)}(t)$ and $\Pi_{\alpha}^{(n)}(t)$}

In this subsection we provide bounds for the $L^{s}$ norms of $P_{\alpha}^{(n)}(t)$ and $\Pi_{\alpha}^{(n)}(t)$ based on the elliptic systems (3.4.6) and (3.4.7). We first expand 
$P_{\alpha}^{(n)}(x, t)$ in terms of singular integral operators,

$$
\begin{aligned}
& P_{\alpha}^{(n)}(x, t)=\int_{\mathbb{R}^{3}} \frac{1}{|x-y|} \Delta P_{\alpha}^{(n)}(y, t) d y \\
& =\int_{\mathbb{R}^{3}}\left[\partial_{l}\left(a_{l k}^{11} \partial_{k} P_{\alpha}^{(n)}+\left(a_{l k}^{12} \partial_{k} \Pi_{\alpha}^{(n)}\right)+\tilde{c}_{l}^{11} \partial_{l} P_{\alpha}^{(n)}+\tilde{c}_{l}^{12} \partial_{l} \Pi_{\alpha}^{(n)}\right] \frac{d y}{|x-y|}\right. \\
& +\int_{\mathbb{R}^{3}} b_{i k}^{11} \partial_{k}\left(b_{j l}^{11} \partial_{l}\left(U_{\alpha, i}^{(n)} U_{\alpha, j}^{(n)}-V_{\alpha, i}^{(n)} V_{\alpha, j}^{(n)}\right)+b_{j l}^{12} \partial_{l}\left(2 U_{\alpha, i}^{(n)} V_{\alpha, j}^{(n)}\right)\right) \frac{d y}{|x-y|} \\
& +\int_{\mathbb{R}^{3}} b_{i k}^{12} \partial_{k}\left(b_{j l}^{21} \partial_{l}\left(U_{\alpha, i}^{(n)} U_{\alpha, j}^{(n)}-V_{\alpha, i}^{(n)} V_{\alpha, j}^{(n)}\right)+b_{j l}^{22} \partial_{l}\left(2 U_{\alpha, i}^{(n)} V_{\alpha, j}^{(n)}\right)\right) \frac{d y}{|x-y|} .
\end{aligned}
$$

As $t$ is fixed, it is suppressed throughout the remainder of this subsection. Label the first line of the last term of the above equation by $I$ and the remaining two lines by $J$. Integrating by parts, $I$ can be written so that no derivatives fall on $P_{\alpha}^{(n)}$ and $\Pi_{\alpha}^{(n)}$,

$$
\begin{aligned}
I= & \int_{\mathbb{R}^{3}} \partial_{k} \partial_{l}\left(\frac{1}{|x-y|}\right)\left[a_{l k}^{11} P_{\alpha}^{(n)}+a_{l k}^{12} \Pi_{\alpha}^{(n)}\right](y) d y \\
& +\int_{\mathbb{R}^{3}} \partial_{l}\left(\frac{1}{|x-y|}\right)\left[\left(\partial_{k} a_{l k}^{11}\right) P_{\alpha}^{(n)}+\left(\partial_{k} a_{l k}^{12}\right) \Pi_{\alpha}^{(n)}\right](y) d y \\
= & I_{1}+I_{2} .
\end{aligned}
$$

The singular integral kernel of $I_{1}$ is of $C Z$-type and we obtain for any $r \in(1, \infty)$ that,

$$
\begin{aligned}
\left\|I_{1}\right\|_{L^{r}} & \leq C_{r}\left(\left\|a_{l k}^{11} P_{\alpha}^{(n)}\right\|_{L^{r}}+\left\|a_{l k}^{12} \Pi_{\alpha}^{(n)}\right\|_{L^{r}}\right) \\
& \leq C_{r}\left\|a_{l k}^{i j}\right\|_{L^{\infty}}\left(\left\|P_{\alpha}^{(n)}\right\|_{L^{r}}+\left\|\Pi_{\alpha}^{(n)}\right\|_{L^{r}}\right) \\
& \leq \frac{C|\alpha| t}{r_{*}}\left(\left\|P_{\alpha}^{(n)}\right\|_{L^{s}}+\left\|\Pi_{\alpha}^{(n)}\right\|_{L^{s}}\right) .
\end{aligned}
$$

For our purposes $s=q / 2>3 / 2$ and, upon investigating the proof of Theorem 3 contained in Section 4 of Chapter II of [101], we can bound $C_{s}$ independently of $s$. Then, provided $|\alpha|$ is sufficiently small, we obtain,

$$
\left\|I_{1}\right\|_{L^{s}} \leq \frac{1}{4}\left(\left\|P_{\alpha}^{(n)}\right\|_{L^{s}}+\left\|\Pi_{\alpha}^{(n)}\right\|_{L^{s}}\right) .
$$

For $I_{2}$ we observe that, since the support of $a_{l j}^{i j}$ is a subset of $2 B_{*}$ and, in the worst case, its gradients have magnitudes comparable to 
$r_{*}^{-1}$, and, again, this is compensated for by making $C_{0}$ large and $|\alpha| t$ correspondingly small to obtain,

$$
\left\|I_{2}\right\|_{L^{s}} \leq \frac{1}{4}\left(\left\|P_{\alpha}^{(n)}\right\|_{L^{s}}+\left\|\Pi_{\alpha}^{(n)}\right\|_{L^{s}}\right)
$$

For $J$, we similarly apply integration by parts to obtain a formula wherein all derivatives fall on the coefficient functions, the singular kernels, or a combination of the two. When all derivatives fall on the singular kernel we obtain via the CZ theory a bound in terms of the $L^{2 s}$ norms of $U_{\alpha}^{(n)}$ and $V_{\alpha}^{(n)}$. In cases where a derivative falls on the coefficient functions the fact that $\nabla b_{i j}^{k l}$ is supported in $2 B_{*}$ allows us to obtain similar bounds.

Letting $s=q / 2$ we ultimately conclude that,

$$
\left\|P_{\alpha}^{(n)}\right\|_{L^{q / 2}} \leq C\left(\left\|U_{\alpha}^{(n)}\right\|_{L^{q}}^{2}+\left\|V_{\alpha}^{(n)}\right\|_{L^{q}}^{2}\right),
$$

and, based on a procedurally identical argument, that,

$$
\left\|\Pi_{\alpha}^{(n)}\right\|_{L^{q / 2}} \leq C\left(\left\|U_{\alpha}^{(n)}\right\|_{L^{q}}^{2}+\left\|V_{\alpha}^{(n)}\right\|_{L^{q}}^{2}\right) .
$$

Noting that the formulas for $P_{\alpha}^{(n)}$ and $\Pi_{\alpha}^{(n)}$ are of convolution type, a similar argument holds for gradients. This ultimately yields,

$$
\left\|\nabla P_{\alpha}^{(n)}\right\|_{L^{q / 2}} \leq C\left(\left\|U_{\alpha}^{(n)}\right\|_{L^{q}}+\left\|V_{\alpha}^{(n)}\right\|_{L^{q}}\right)\left(\left\|\nabla U_{\alpha}^{(n)}\right\|_{L^{q}}+\left\|\nabla V_{\alpha}^{(n)}\right\|_{L^{q}}\right),
$$

and,

$$
\left\|\nabla \Pi_{\alpha}^{(n)}\right\|_{L^{q / 2}} \leq C\left(\left\|U_{\alpha}^{(n)}\right\|_{L^{q}}+\left\|V_{\alpha}^{(n)}\right\|_{L^{q}}\right)\left(\left\|\nabla U_{\alpha}^{(n)}\right\|_{L^{q}}+\left\|\nabla V_{\alpha}^{(n)}\right\|_{L^{q}}\right) .
$$

\subsubsection{Inductive estimates in $L^{q}$ for $U_{\alpha}^{(n)}$ and $V_{\alpha}^{(n)}$}

This subsection is dedicated to showing that on some time interval $\left[0, T_{1}\right]$ and for sufficiently small values of $|\alpha|$, the $L^{q}$ norms of $U_{\alpha}^{(n)}$ and $V_{\alpha}^{(n)}$ are bounded solely in terms of the initial data and the complexified forcing. To save space, when formally similar estimates apply across a variety of terms we omit the details in all but a single illustrative case. For ease of reading we break our treatment into a base case when $n=1$ and an inductive step for general $n \in \mathbb{N}$. 
Base case: Applying Duhamel's formula in the context of (3.4.4) allows us to express $U_{\alpha}^{(1)}$ in integral form as,

$$
\begin{aligned}
U_{\alpha}^{(1)}(t)= & e^{t \Delta} u_{0}+\int_{0}^{t} e^{(t-\tau) \Delta} F_{\alpha}(\tau) d \tau \\
& +\int_{0}^{t} e^{(t-\tau) \Delta}\left[\alpha_{l} c_{l m}^{11} \partial_{m} U_{\alpha}^{(1)}+\alpha_{l} c_{l m}^{12} \partial_{m} V_{\alpha}^{(1)}\right](\tau) d \tau \\
& +\int_{0}^{t} e^{(t-\tau) \Delta}\left[\partial_{l}\left(a_{l k}^{11} \partial_{k} U_{\alpha}^{(1)}\right)+\partial_{l}\left(a_{l k}^{12} \partial_{k} V_{\alpha}^{(1)}\right)\right](\tau) d \tau \\
& +\int_{0}^{t} e^{(t-\tau) \Delta}\left[+\tilde{c}_{l}^{11} \partial_{l} U_{\alpha}^{(1)}+\tilde{c}_{l}^{12} \partial_{l} V_{\alpha}^{(1)}\right](\tau) d \tau .
\end{aligned}
$$

Consider a fixed time $t$ and apply the $L^{q}$ norm throughout the integral equation. Then, the terms involving the initial data and $F_{\alpha}$ satisfy,

$$
\left\|e^{t \Delta} u_{0}\right\|_{L^{q}} \leq C\left\|u_{0}\right\|_{L^{q}}
$$

and,

$$
\left\|\int_{0}^{t} e^{(t-\tau) \Delta} F_{\alpha}(\tau) d \tau\right\|_{L^{q}} \leq C T \sup _{0<\tau<T}\left\|F_{\alpha}(\tau)\right\|_{L^{q}}
$$

while the remaining terms are bounded via classical techniques (see, for instance, inequalities (2.3) through (2.5') in [72]). For the first order terms we apply Young's convolution inequality and Hölder's inequality. Assuming $T^{1 / 2} \leq r_{*}$ this leads to estimates along the lines of,

$$
\left\|\int_{0}^{t} e^{(t-\tau) \Delta} \alpha_{l} c_{l m}^{11} \partial_{m} U_{\alpha}^{(1)}(\tau) d \tau\right\|_{L^{q}} \leq C|\alpha| T^{\frac{r-1}{r}}\left(\int_{0}^{T}\left\|\nabla U_{\alpha}^{(1)}(t)\right\|_{L^{q}}^{r} d t\right)^{\frac{1}{r}},
$$

where $r>2$ is a fixed exponent (see the next inequality).

For the second order terms we move the derivative to the Gaussian kernel (this also introduces a lower order term but, as this ultimately satisfies the same estimate, we exclude it from consideration). Again using the bounds from [72] and further stipulating that,

$$
r>\frac{2 q}{q-3}
$$

we obtain,

$$
\left\|\int_{0}^{t} \partial_{l} e^{(t-\tau) \Delta} a_{l k}^{11} \partial_{k} U_{\alpha}^{(1)}(\tau) d \tau\right\|_{L^{q}} \leq C|\alpha| T^{\frac{r-1}{r}}\left(\int_{0}^{T}\left\|\nabla U_{\alpha}^{(1)}(t)\right\|_{L^{q}}^{r} d t\right)^{\frac{1}{r}}
$$


Taken together with similar estimates for $V_{\alpha}^{(1)}$ we obtain an initial $L^{q}$ estimate,

$$
\begin{aligned}
& \sup _{0<t<T}\left\|U_{\alpha}^{(1)}(t)\right\|_{L^{q}}+\sup _{0<t<T}\left\|V_{\alpha}^{(1)}(t)\right\|_{L^{q}} \\
& \leq C\left\|u_{0}\right\|_{L^{q}}+C T\left(\sup _{0<\tau<T}\left\|F_{\alpha}(\tau)\right\|_{L^{q}}+\sup _{0<\tau<T}\left\|G_{\alpha}(\tau)\right\|_{L^{q}}\right) \\
& +C|\alpha| T^{\frac{r-1}{r}}\left[\left(\int_{0}^{T}\left\|\nabla U_{\alpha}^{(1)}(t)\right\|_{L^{q}}^{r} d t\right)^{\frac{1}{r}}+\left(\int_{0}^{T}\left\|\nabla V_{\alpha}^{(1)}(t)\right\|_{L^{q}}^{r} d t\right)^{\frac{1}{r}}\right] .
\end{aligned}
$$

We estimate the time-integrated quantities appearing in (3.4.12) by applying a spatial gradient across the integral equation for $U_{\alpha}^{(1)}(t)$ noting that, since $e^{t \Delta}$ is a convolution operator, this extra gradient can be put on the Gaussian kernel when appropriate. For the terms involving the initial data and forcing we have,

$$
\left(\int_{0}^{T}\left\|e^{t \Delta} \nabla u_{0}\right\|_{L^{q}}^{r} d t\right)^{\frac{1}{r}} \leq C T^{1 / r}\left\|\nabla u_{0}\right\|_{L^{q}}
$$

and,

$$
\begin{aligned}
& \left(\int_{0}^{T}\left\|\int_{0}^{t} e^{(t-\tau) \Delta} \nabla F_{\alpha}(\tau) d \tau\right\|_{L^{q}}^{r} d t\right)^{\frac{1}{r}} \\
& \leq C T\left(\int_{0}^{T}\left\|\nabla F_{\alpha}(\tau)\right\|_{L^{q}}^{r} d t\right)^{\frac{1}{r}} .
\end{aligned}
$$

The first order terms all satisfy the estimate,

$$
\begin{aligned}
& \left(\int_{0}^{T}\left\|\int_{0}^{t} \nabla e^{(t-\tau) \Delta} \alpha_{l} c_{l m}^{11} \partial_{m} U_{\alpha}^{(1)}(\tau) d \tau\right\|_{L^{q}}^{r} d t\right)^{\frac{1}{r}} \\
& \leq C|\alpha| T^{1 / r} \sup _{0<t<T}\left\|U_{\alpha}^{(1)}(t)\right\|_{L^{q}}
\end{aligned}
$$

which can be verified using the standard estimates involving the heat kernel as in [72]. The second order terms are more interesting as the singular kernel in the time variable becomes critical when applying the weak Young's inequality. To get around this we apply the maximal $L^{r}-L^{q}$-regularity of the heat kernel as discussed in [84]. Indeed, based 
on (3.4.2), we obtain,

$$
\begin{aligned}
& \left(\int_{0}^{T}\left\|\int_{0}^{t} \nabla \partial_{l} e^{(t-\tau) \Delta} a_{l k}^{11} \partial_{k} U_{\alpha}^{(1)}(\tau) d \tau\right\|_{L^{q}}^{r} d t\right)^{\frac{1}{r}} \\
& \leq C|\alpha| T^{1 / 2}\left(\int_{0}^{T}\left\|\partial_{k} U_{\alpha}^{(1)}(t)\right\|_{L^{q}}^{r} d t\right)^{\frac{1}{r}}
\end{aligned}
$$

where we have appealed to (3.4.2) and the stipulation $T \leq r_{*}^{2}$.

Combining estimates (3.4.13) through (3.4.16) and noting similar results are valid for $V_{\alpha}^{(1)}$, we see that,

$$
\begin{aligned}
& \left(\int_{0}^{T}\left\|\nabla U_{\alpha}^{(1)}(t)\right\|_{L^{q}}^{r} d t\right)^{\frac{1}{r}}+\left(\int_{0}^{T}\left\|\nabla V_{\alpha}^{(1)}(t)\right\|_{L^{q}}^{r} d t\right)^{\frac{1}{r}} \\
& \leq C T^{1 / r}\left\|\nabla u_{0}\right\|_{L^{q}} \\
& \quad+C T\left[\left(\int_{0}^{T}\left\|\nabla F_{\alpha}(\tau)\right\|_{L^{q}}^{r} d t\right)^{\frac{1}{r}}+\left(\int_{0}^{T}\left\|\nabla G_{\alpha}(\tau)\right\|_{L^{q}}^{r} d t\right)^{\frac{1}{r}}\right] \\
& \quad+C|\alpha| T^{1 / r}\left[\sup _{0<\tau<T}\left\|U_{\alpha}^{(1)}\right\|_{L^{q}}+\sup _{0<\tau<T}\left\|V_{\alpha}^{(1)}\right\|_{L^{q}}\right] \\
& \quad+C|\alpha| T^{1 / 2}\left[\left(\int_{0}^{T}\left\|\nabla U_{\alpha}^{(1)}(t)\right\|_{L^{q}}^{r} d t\right)^{\frac{1}{r}}+\left(\int_{0}^{T}\left\|\nabla V_{\alpha}^{(1)}(t)\right\|_{L^{q}}^{r} d t\right)^{\frac{1}{r}}\right] .
\end{aligned}
$$

By restricting $|\alpha|$ to ensure that,

$$
|\alpha| T^{1 / 2} \leq \frac{1}{2 C_{0}},
$$

we can absorb the last term above into the left hand side of the inequality. Returning to (3.4.12) this leads to an improved estimate for the base case,

$$
\begin{aligned}
& \sup _{0<t<T}\left\|U_{\alpha}^{(1)}(t)\right\|_{L^{q}}+\sup _{0<t<T}\left\|V_{\alpha}^{(1)}(t)\right\|_{L^{q}} \\
& \leq C\left\|u_{0}\right\|_{L^{q}}+C|\alpha| T\left\|\nabla u_{0}\right\|_{L^{q}} \\
& +C T\left(\sup _{0<\tau<T}\left\|F_{\alpha}(\tau)\right\|_{L^{q}}+\sup _{0<\tau<T}\left\|G_{\alpha}(\tau)\right\|_{L^{q}}\right) \\
& +C|\alpha| T^{\frac{2 r-1}{r}}\left[\left(\int_{0}^{T}\left\|\nabla F_{\alpha}(\tau)\right\|_{L^{q}}^{r} d t\right)^{\frac{1}{r}}+\left(\int_{0}^{T}\left\|\nabla G_{\alpha}(\tau)\right\|_{L^{q}}^{r} d t\right)^{\frac{1}{r}}\right] \\
& +C|\alpha|^{2} T\left[\sup _{0<\tau<T}\left\|U_{\alpha}^{(1)}\right\|_{L^{q}}+\sup _{0<\tau<T}\left\|V_{\alpha}^{(1)}\right\|_{L^{q}}\right]
\end{aligned}
$$


the last term of which can be absorbed into the left hand side in virtue of (3.4.17). Using the labels,

$$
\begin{aligned}
& M_{0}=C\left\|u_{0}\right\|_{L^{q}}, \\
& M_{0}^{\prime}=C\left\|\nabla u_{0}\right\|_{L^{q}}, \\
& M_{f}=C \sup _{\alpha \in S_{f}}\left[\sup _{0<\tau<T_{0}}\left\|F_{\alpha}(\tau)\right\|_{L^{q}}+\sup _{0<\tau<T_{0}}\left\|G_{\alpha}(\tau)\right\|_{L^{q}}\right], \\
& M_{f}^{\prime}=C \sup _{\alpha \in S_{f}}\left[\left(\int_{0}^{T}\left\|\nabla F_{\alpha}(\tau)\right\|_{L^{q}}^{r} d t\right)^{\frac{1}{r}}+\left(\int_{0}^{T}\left\|\nabla G_{\alpha}(\tau)\right\|_{L^{q}}^{r} d t\right)^{\frac{1}{r}}\right],
\end{aligned}
$$

the previous estimate can be reformulated as,

$$
\begin{aligned}
& \sup _{0<t<T}\left\|U_{\alpha}^{(1)}(t)\right\|_{L^{q}}+\sup _{0<t<T}\left\|V_{\alpha}^{(1)}(t)\right\|_{L^{q}} \\
& \leq M_{0}+T^{1 / 2} M_{0}^{\prime}+T M_{f}+T^{(3 r-2) /(2 r)} M_{f}^{\prime} .
\end{aligned}
$$

Thus, fixing a time $T_{0}$ so that,

$$
T_{0} \leq \min \left\{r_{*}^{2},\left(\frac{M_{0}}{M_{0}^{\prime}}\right)^{2},\left(\frac{M_{0}}{M_{f}}\right),\left(\frac{M_{0}}{M_{f}^{\prime}}\right)^{\frac{2 r}{3 r-2}}\right\},
$$

guarantees that for $T \leq T_{0}$ we have,

$$
\sup _{0<t<T}\left\|U_{\alpha}^{(1)}(t)\right\|_{L^{q}}+\sup _{0<t<T}\left\|V_{\alpha}^{(1)}(t)\right\|_{L^{q}} \leq 4 M_{0},
$$

provided $\alpha$ is chosen in accordance with (3.4.17). This also leads to,

$$
\begin{aligned}
& \left(\int_{0}^{T}\left\|\nabla U_{\alpha}^{(1)}(t)\right\|_{L^{q}}^{r} d t\right)^{\frac{1}{r}}+\left(\int_{0}^{T}\left\|\nabla V_{\alpha}^{(1)}(t)\right\|_{L^{q}}^{r} d t\right)^{\frac{1}{r}} \\
& \leq T^{1 / r} M_{0}^{\prime}+T M_{f}^{\prime}+4 C|\alpha| T^{1 / r} M_{0},
\end{aligned}
$$

from which, considering (3.4.20), we obtain,

$$
\begin{aligned}
& T^{(r-2) /(2 r)}\left[\left(\int_{0}^{T}\left\|\nabla U_{\alpha}^{(1)}(t)\right\|_{L^{q}}^{r} d t\right)^{\frac{1}{r}}+\left(\int_{0}^{T}\left\|\nabla V_{\alpha}^{(1)}(t)\right\|_{L^{q}}^{r} d t\right)_{(3.4 .}^{\frac{1}{r}}\right] \\
& \leq M_{0}+T^{1 / 2} M_{0}^{\prime}+T^{(3 r-2) /(2 r)} M_{f}^{\prime} \\
& \leq 4 M_{0} .
\end{aligned}
$$


Inductive step: The foundation for the inductive argument reflects inequalities (3.4.21) and (3.4.23). Indeed, we aim to show that there exists a time $T_{1}$ in $\left(0, T_{0}\right]$ so that if, for all $T \in\left(0, T_{1}\right)$ and some $n \in \mathbb{N}$, the inequalities,

$$
\sup _{0<t<T}\left\|U_{\alpha}^{(n)}(t)\right\|_{L^{q}}+\sup _{0<t<T}\left\|V_{\alpha}^{(n)}(t)\right\|_{L^{q}} \leq 4 M_{0},
$$

and,

$$
\begin{aligned}
& T^{\frac{r-2}{2 r}}\left[\left(\int_{0}^{T}\left\|\nabla U_{\alpha}^{(n)}(t)\right\|_{L^{q}}^{r} d t\right)^{\frac{1}{r}}+\left(\int_{0}^{T}\left\|\nabla V_{\alpha}^{(n)}(t)\right\|_{L^{q}}^{r} d t\right)^{\frac{1}{r}}\right] \\
& \leq 4 M_{0},
\end{aligned}
$$

are satisfied, then so are the inequalities,

$$
\sup _{0<t<T}\left\|U_{\alpha}^{(n+1)}(t)\right\|_{L^{q}}+\sup _{0<t<T}\left\|V_{\alpha}^{(n+1)}(t)\right\|_{L^{q}} \leq 4 M_{0},
$$

and,

$$
\begin{aligned}
& T^{\frac{r-2}{2 r}}\left[\left(\int_{0}^{T}\left\|\nabla U_{\alpha}^{(n+1)}(t)\right\|_{L^{q}}^{r} d t\right)^{\frac{1}{r}}+\left(\int_{0}^{T}\left\|\nabla V_{\alpha}^{(n+1)}(t)\right\|_{L^{q}}^{r} d t\right)^{\frac{1}{r}}\right] \\
& \leq 4 M_{0},
\end{aligned}
$$

provided $|\alpha|$ is appropriately controlled in terms of $T$ and $r_{*}$.

Our starting point is, again, Duhamel's principle which leads us to 
the integral formula,

$$
\begin{aligned}
U_{\alpha}^{(n+1)}(t)= & e^{t \Delta} u_{0}+\int_{0}^{t} e^{(t-\tau) \Delta} F_{\alpha}(\tau) d \tau \\
& +\int_{0}^{t} e^{(t-\tau) \Delta}\left[\alpha_{l} c_{l m}^{11} \partial_{m} U_{\alpha}^{(n+1)}+\alpha_{l} c_{l m}^{12} \partial_{m} V_{\alpha}^{(n+1)}\right](\tau) d \tau \\
& +\int_{0}^{t} e^{(t-\tau) \Delta}\left[\partial_{l}\left(a_{l k}^{11} \partial_{k} U_{\alpha}^{(n+1)}\right)+\partial_{l}\left(a_{l k}^{12} \partial_{k} V_{\alpha}^{(n+1)}\right)\right](\tau) d \tau \\
& +\int_{0}^{t} e^{(t-\tau) \Delta}\left[+\tilde{c}_{l}^{11} \partial_{l} U_{\alpha}^{(n+1)}+\tilde{c}_{l}^{12} \partial_{l} V_{\alpha}^{(n+1)}\right](\tau) d \tau \\
& -\int_{0}^{t} e^{(t-\tau) \Delta}\left[U_{\alpha, l}^{(n)}\left[b_{l k}^{11} \partial_{k} U_{\alpha}^{(n)}+b_{l k}^{12} \partial_{k} V_{\alpha}^{(n)}\right]\right](\tau) d \tau \\
& +\int_{0}^{t} e^{(t-\tau) \Delta}\left[V_{\alpha, l}^{(n)}\left[b_{l k}^{21} \partial_{k} U_{\alpha}^{(n)}+b_{l k}^{22} \partial_{k} V_{\alpha}^{(n)}\right]\right](\tau) d \tau \\
& -\int_{0}^{t} e^{(t-\tau) \Delta}\left[b_{l k}^{11} \partial_{k} P_{\alpha}^{(n)}+b_{l k}^{12} \partial_{k} \Pi_{\alpha}^{(n)}\right](\tau) d \tau
\end{aligned}
$$

The terms from the first three lines, namely those involving the initial data, the forcing, and the $(n+1)$-level linear terms, all satisfy estimates analogous to those established for the base case. The only new estimates required for the inductive step involve the terms from the remaining lines and we turn our attention to these.

To illustrate our approach for the local $(n)$-level terms we follow the approach of [72] and obtain,

$$
\begin{aligned}
& \left\|\int_{0}^{t} e^{(t-\tau) \Delta} U_{\alpha, l}^{(n)} b_{l k}^{11} \partial_{k} U_{\alpha}^{(n)}(\tau) d \tau\right\|_{L^{q}} \\
& \leq C T^{(r-1) / r-3 /(2 q)} \sup _{0<\tau<T}\left\|U_{\alpha}^{(n)}(\tau)\right\|_{L^{q}}\left(\int_{0}^{t}\left\|\nabla U_{\alpha}^{(n)}(\tau)\right\|_{L^{q}}^{r} d \tau\right)^{\frac{1}{r}},
\end{aligned}
$$

For the $L^{r}-L^{q}$ estimates involving an extra derivative we similarly obtain bounds along the lines of,

$$
\begin{aligned}
& \left(\int_{0}^{T}\left\|\int_{0}^{t} \nabla e^{(t-\tau) \Delta} U_{\alpha, l}^{(n)} b_{l k}^{11} \partial_{k} U_{\alpha}^{(n)}(\tau) d \tau\right\|_{L^{q}}^{r} d t\right)^{\frac{1}{r}} \\
& \leq C T^{(q-3) /(2 q)} \sup _{0<\tau<T}\left\|U_{\alpha}^{(n)}(\tau)\right\|_{L^{q}}\left(\int_{0}^{T}\left\|\nabla U_{\alpha}\right\|_{L^{q}}^{r} d t\right)^{\frac{1}{r}} .
\end{aligned}
$$


The $(n)$-level terms associated with the pressure are dealt with using the estimates established in subsection 3.4.1 and are achieved similarly to (3.4.28) and (3.4.29). Focusing on $P_{\alpha}^{(n)}$, for example, and applying (3.4.10), we see,

$$
\begin{aligned}
& \left\|\int_{0}^{t} e^{(t-\tau) \Delta} b_{l k}^{11} \partial_{k} P_{\alpha}^{(n)}(\tau) d \tau\right\|_{L^{q}} \\
& \leq C T^{(r-1) / r-3 /(2 q)}\left(\sup _{0<\tau<T}\left\|U_{\alpha}^{(n)}(\tau)\right\|_{L^{q}}+\sup _{0<\tau<T}\left\|V_{\alpha}^{(n)}(\tau)\right\|_{L^{q}}\right) \\
& \cdot\left[\left(\int_{0}^{T}\left\|\nabla U_{\alpha}^{(n)}(\tau)\right\|_{L^{q}}^{r} d \tau\right)^{\frac{1}{r}}+\left(\int_{0}^{T}\left\|\nabla V_{\alpha}^{(n)}(\tau)\right\|_{L^{q}}^{r} d \tau\right)^{\frac{1}{r}}\right],
\end{aligned}
$$

and,

$$
\begin{aligned}
& \left(\int_{0}^{T}\left\|\int_{0}^{t} \nabla e^{(t-\tau) \Delta} b_{l k}^{11} \partial_{k} P_{\alpha}^{(n)}(\tau) d \tau\right\|_{L^{q}}^{r} d t\right)^{1 / r} \\
& \leq C T^{(q-3) /(2 q)}\left(\sup _{0<\tau<T}\left\|U_{\alpha}^{(n)}(\tau)\right\|_{L^{q}}+\sup _{0<\tau<T}\left\|V_{\alpha}^{(n)}(\tau)\right\|_{L^{q}}\right) \\
& \cdot\left[\left(\int_{0}^{T}\left\|\nabla U_{\alpha}^{(n)}(\tau)\right\|_{L^{q}}^{r} d \tau\right)^{\frac{1}{r}}+\left(\int_{0}^{T}\left\|\nabla V_{\alpha}^{(n)}(\tau)\right\|_{L^{q}}^{r} d \tau\right)^{\frac{1}{r}}\right] .
\end{aligned}
$$

With these estimates in mind we are ready to establish inequalities (3.4.26) and (3.4.27) which will conclude our inductive argument. We first consider (3.4.27) observing that,

$$
\begin{aligned}
& \left(\int_{0}^{T}\left\|\nabla U_{\alpha}^{(n+1)}(t)\right\|_{L^{q}}^{r} d t\right)^{\frac{1}{r}}+\left(\int_{0}^{T}\left\|\nabla V_{\alpha}^{(n+1)}(t)\right\|_{L^{q}}^{r} d t\right)^{\frac{1}{r}} \\
& \leq C T^{1 / r}\left\|\nabla u_{0}\right\|_{L^{q}} \\
& +C T\left[\left(\int_{0}^{T}\left\|\nabla F_{\alpha}(\tau)\right\|_{L^{q}}^{r} d t\right)^{\frac{1}{r}}+\left(\int_{0}^{T}\left\|\nabla G_{\alpha}(\tau)\right\|_{L^{q}}^{r} d t\right)^{\frac{1}{r}}\right] \\
& +C|\alpha| T^{1 / r}\left[\sup _{0<\tau<T}\left\|U_{\alpha}^{(n+1)}(\tau)\right\|_{L^{q}}+\sup _{0<\tau<T}\left\|V_{\alpha}^{(n+1)}(\tau)\right\|_{L^{q}}\right] \\
& +C|\alpha| T^{\frac{1}{2}}\left[\left(\int_{0}^{T}\left\|\nabla U_{\alpha}^{(n+1)}(t)\right\|_{L^{q}}^{r} d t\right)^{\frac{1}{r}}+\left(\int_{0}^{T}\left\|\nabla V_{\alpha}^{(n+1)}(t)\right\|_{L^{q}}^{r} d t\right)^{\frac{1}{r}}\right] \\
& +C T^{(q-3) /(2 q)}\left(\sup _{0<\tau<T}\left\|U_{\alpha}^{(n)}(\tau)\right\|_{L^{q}}+\sup _{0<\tau<T}\left\|V_{\alpha}^{(n)}(\tau)\right\|_{L^{q}}\right) \\
& \cdot\left[\left(\int_{0}^{T}\left\|\nabla U_{\alpha}^{(n)}(\tau)\right\|_{L^{q}}^{r} d \tau\right)^{\frac{1}{r}}+\left(\int_{0}^{T}\left\|\nabla V_{\alpha}^{(n)}(\tau)\right\|_{L^{q}}^{r} d \tau\right)^{\frac{1}{r}}\right] .
\end{aligned}
$$


Provided $T \leq T_{0}$ this can be simplified using (3.4.17), the procedures illustrated in our treatment of the base case, and the inductive hypothesis, to ultimately yield,

$$
\begin{aligned}
& \left(\int_{0}^{T}\left\|\nabla U_{\alpha}^{(n+1)}(t)\right\|_{L^{q}}^{r} d t\right)^{\frac{1}{r}}+\left(\int_{0}^{T}\left\|\nabla V_{\alpha}^{(n+1)}(t)\right\|_{L^{q}}^{r} d t\right)^{\frac{1}{r}} \\
& \leq T^{1 / r} M_{0}^{\prime}+T M_{f}^{\prime}+T^{(q-3) /(2 q)-(r-2) /(2 r)} M_{0}^{2} \\
& \quad+C|\alpha| T^{1 / r}\left[\sup _{0<\tau<T}\left\|U_{\alpha}^{(n+1)}\right\|_{L^{q}}+\sup _{0<\tau<T}\left\|V_{\alpha}^{(n+1)}\right\|_{L^{q}}\right] .
\end{aligned}
$$

This estimate will lead to (3.4.27) after we establish (3.4.26). Toward this we first observe that,

$$
\begin{aligned}
\sup _{0<t<T} & \left\|U_{\alpha}^{(n+1)}(t)\right\|_{L^{q}}+\sup _{0<t<T}\left\|V_{\alpha}^{(n+1)}(t)\right\|_{L^{q}} \\
\leq & C\left\|u_{0}\right\|_{L^{q}}+C T\left(\sup _{0<\tau<T}\left\|F_{\alpha}(\tau)\right\|_{L^{q}}+\sup _{0<\tau<T}\left\|G_{\alpha}(\tau)\right\|_{L^{q}}\right) \\
+ & C|\alpha| T^{(r-1) / r}\left[\left(\int_{0}^{T}\left(\left\|\nabla U_{\alpha}^{(n+1)}(t)\right\|_{L^{q}}\right)^{r} d t\right)^{\frac{1}{r}}\right. \\
+ & \left.\left(\int_{0}^{T}\left(\left\|\nabla V_{\alpha}^{(n+1)}(t)\right\|_{L^{q}}\right)^{r} d t\right)^{\frac{1}{r}}\right] \\
+ & C T^{(r-1) / r-3 /(2 q)}\left(\sup _{0<\tau<T}\left\|U_{\alpha}^{(n)}(\tau)\right\|_{L^{q}}+\sup _{0<\tau<T}\left\|V_{\alpha}^{(n)}(\tau)\right\|_{L^{q}}\right) \\
\cdot & {\left[\left(\int_{0}^{T}\left\|\nabla U_{\alpha}^{(n)}(\tau)\right\|_{L^{q}}^{r} d \tau\right)^{\frac{1}{r}}+\left(\int_{0}^{T}\left\|\nabla V_{\alpha}^{(n)}(\tau)\right\|_{L^{q}}^{r} d \tau\right)^{\frac{1}{r}}\right] . }
\end{aligned}
$$

Using the inductive hypothesis, condition (3.4.17), and the formula for the time integrated quantity, we see that,

$$
\begin{aligned}
& \sup _{0<t<T}\left\|U_{\alpha}^{(n+1)}(t)\right\|_{L^{q}}+\sup _{0<t<T}\left\|V_{\alpha}^{(n+1)}(t)\right\|_{L^{q}} \\
& \leq M_{0}+T M_{f}+T^{(q-3) /(2 q)} M_{0}^{2}+T^{1 / 2} M_{0}^{\prime}+T^{(3 r-2) /(2 r)} M_{f}^{\prime} .
\end{aligned}
$$

Then, assuming that,

$$
T_{1} \leq \min \left\{T_{0},\left(\frac{1}{C_{0} M_{0}}\right)^{\frac{2 q}{q-3}}\right\}
$$


we obtain inequality (3.4.26) for $T \in\left(0, T_{1}\right]$, i.e.,

$$
\sup _{0<t<T}\left\|U_{\alpha}^{(n+1)}(t)\right\|_{L^{q}}+\sup _{0<t<T}\left\|V_{\alpha}^{(n+1)}(t)\right\|_{L^{q}} \leq 4 M_{0} .
$$

We also obtain (3.4.27) since,

$$
\begin{aligned}
& T^{(r-2) /(2 r)}\left(\int_{0}^{T}\left\|\nabla U_{\alpha}^{(n+1)}(t)\right\|_{L^{q}}^{r} d t\right)^{\frac{1}{r}}+\left(\int_{0}^{T}\left\|\nabla V_{\alpha}^{(n+1)}(t)\right\|_{L^{q}}^{r} d t\right)^{\frac{1}{r}} \\
& \leq T^{1 / 2} M_{0}^{\prime}+T^{(3 r-2) /(2 r)} M_{f}^{\prime}+T^{(q-3) /(2 q)} M_{0}^{2}+M_{0} \\
& \leq 4 M_{0},
\end{aligned}
$$

provided $T$ is still taken in $\left(0, T_{1}\right]$.

\subsubsection{Proof of Theorem 3.4.1}

For $t \in\left(0, T_{1}\right]$, let,

$$
\Omega(t)=\bigcup_{\alpha \in S_{f} ;|\alpha| t^{1 / 2} \leq\left(2 C_{0}\right)^{-1}}\left\{x+i y \in \mathbb{C}^{3}: x \in 2 B_{*}, y=\alpha \psi(x) t\right\} .
$$

The estimates from the previous section ensure that for all $t \in\left(0, T_{1}\right]$ and $n \in \mathbb{N}$ we have the uniform bound,

$$
\int_{\Omega(t)}\left|\left(u^{(n)}+i v^{(n)}\right)(x, y)\right|^{q} d x d y \leq\left(\frac{2 T_{1}^{1 / 2}}{C_{0}}\right)^{3} M_{0},
$$

which, referring to Lemma 2.4 of [62], implies that $\left\{u^{(n)}+i v^{(n)}\right\}_{n \in \mathbb{N}}$ belongs to a normal family. This allows us to extract subsequences which, following the argument contained in [62], converge to an analytic function $u+i v$ on compact sets contained in $\Omega(t)$ such as the closure of the domain $\Omega_{*}(t)$ which was specified in the statement of the theorem; the details are omitted here to avoid technical redundancy. Assuming the premises of Theorem 3.4.1 and taking $T_{1}$ to be the value identified in the previous subsection, we conclude that there exists a solution $u$ belonging to $C\left(0, T_{1} ; L^{q}\left(\mathbb{R}^{3}\right)\right)$ which agrees with the restriction to $B_{*}$ of a function $U+i V$ which, for $t \in\left(0, T_{1}\right]$, is analytic on $\Omega_{*}(t)$ and satisfies the complexified Navier-Stokes equations locally on the parabolic domain,

$$
\left\{(x+i y, t) \in \mathbb{C}^{3} \times\left(0, T_{1}\right]: x+i y \in \Omega_{*}(t)\right\} .
$$




\section{Chapter 4}

\section{Logarithmically subcritical scenarios for solutions to the 3D Navier-Stokes equations}

\subsection{Introduction}

In this chapter we describe certain scenarios wherein locally smooth weak solutions to 3D NSE behave subcritically with respect to the critical scaling of one-dimensional local sparseness. The context of our work reflects two complementary publications, [44, 61], where a new dynamic approach to bridging the scaling gap is elucidated. The physical motivation is the persistence (in the average sense) of the axial lengths of vortex filaments. This picture is supported in experimental and numerical studies and by a mathematical result given in [44]. By considering this persistence and its implications for the decay rate of the volume of the region of intense vorticity, a connection is found between the scaling of the latter quantity and the critical scaling of one-dimensional local sparseness. As this argument provides the context for our own results we take a moment to make the matter more precise.

The rigorous regularity criteria is presented in [61] and identifies the critical scaling for the local one-dimensional sparseness of the region of intense vorticity to be asymptotically comparable to $c_{0}\|\omega(t)\|_{\infty}^{-1 / 2}$. The proof of this geometric measure-type regularity criteria is based on an interplay between the diffusion, the basic symmetries present in the 3D NSE, and geometric properties of the harmonic measure which results 
in strong anisotropic diffusion (note that the contents of Section 3.3 illustrate this argument in the context of MHD). On the other hand, the a priori estimates on the $L^{1}$ norm of the vorticity found in $[31,81]$ imply the volume of the region where vorticity magnitudes are high decays according to,

$$
\operatorname{Vol}\left(\Lambda_{t}\left(\frac{1}{c_{1}}\|\omega(t)\|_{\infty}\right)\right) \leq \frac{c_{2}}{\|\omega(t)\|_{\infty}},
$$

where $\Lambda_{t}(y)=\{x:|\omega(x, t)| \geq y\}$. Interestingly, if the region of intense vorticity corresponds to the space occupied by filamentary vortex structures and the length of these filaments is non-decreasing - as would be the case, for instance, if they are pinned to the characteristic length scale of a turbulent volume - then the anti-axial diameters of these filaments exhibit a rate of decay of order at least $c_{3}\|\omega(t)\|_{\infty}^{-1 / 2}$. This matches the critical scaling for local one-dimensional sparseness and it is in this asymptotic sense that the problem is rendered critical. That the vortex filaments have persistent lengths (in the average) which are comparable to the scale of the turbulent region is not yet rigorously established but is supported by numerical evidence as well as a mathematical evidence contained in [44].

The soundness of this argument is not by itself sufficient to trigger a regularity result as additional work is required to break criticality (the issue lies in comparing $c_{0}$ and $c_{3}$ ). In this chapter we show that this can be partially overcome by illustrating two scenarios in which the region of intense vorticity decays at a faster rate than the critical rate discussed in [44, 61], thereby rendering the constants irrelevant. We note for clarity that these are not stated as regularity criteria but instead scenarios under which 3D NSE becomes subcritical with regard to the scaling described in $[44,61]$.

The first strategy is detailed in Section 4.3 where we are interested in concluding,

$$
\operatorname{Vol}\left(\Lambda_{t}\left(\frac{1}{c_{1}}\|\omega(t)\|_{\infty}\right)\right) \leq \frac{c_{3}}{\|\omega(t)\|_{\infty} \Phi\left(\|\omega(t)\|_{\infty}\right)},
$$

where $\Phi(x)=\log \left(1+x^{2}\right)$ or $\Phi(x)=\log \log \left(e+x^{2}\right)$. These decay rates will be obtained by imposing certain structural requirements on the blow-up rates exhibited by vorticity components. Let $\omega_{j}^{+}$and $\omega_{j}^{-}$respectively denote the positive and negative vorticity components truncated away from zero. We will define amenable blow-up rates of orders 
0 and 1 generally in Definition 4.3.1 and here only illustrate a class of functions exhibiting such blow-up profiles and describe how the blowup rates are connected to $\omega_{j}^{ \pm}$. A function $g$ (to be identified with one of the $\omega_{j}^{ \pm} \mathrm{s}$ ) exhibits a local algebraic blow-up (around $x_{0}$, at time $T$ ) if there exists a constant $C>1$ so that, for $(x, t)$ in a parabolic cylinder, $Q=B\left(x_{0}, r\right) \times(0, T)$, we have,

$$
\frac{1}{C}\left(\frac{1}{|p(x, t)|+\tau(t)}\right)^{\alpha(t)} \leq|g(x, t)| \leq C\left(\frac{1}{|p(x, t)|+\tau(t)}\right)^{\alpha(t)},
$$

where, at each time $t, p(\cdot, t)$ is some polynomial of degree less than a fixed natural number $d, \alpha$ is positive valued and bounded away from both 0 and $\infty$, and $\tau$ is a positive (up to $T$ ) scalar function of time which vanishes at the singular time. The envisioned blow-up occurs at the zeros of $p(x, T)$ lying within $B\left(x_{0}, r\right)$. There is a considerable amount of freedom present in the above construction as the polynomial is allowed to vary wildly in the time dimension. We will also consider a scenario where some asymmetry is allowed between the bounds assumed on vorticity components. In particular, for appropriate blow-up rates $D_{j}^{ \pm}(x, t)$, we will require,

$$
D_{j}^{ \pm}(x, t) \leq\left|\omega_{j}^{ \pm}(x, t)\right| \leq C D_{j}^{ \pm}(x, t)^{\beta_{j}^{ \pm}(t)},
$$

where $\beta_{j}^{ \pm}:(0, T) \rightarrow\left[1, B_{j}\right]$ for some fixed value $B_{j}$.

A key role is played in our analysis by the cancellations evident in the vortex stretching term in the context of the real Hardy space $\mathcal{H}^{1}$ exploited via the Div-Curl lemma [29] and the $\mathcal{H}^{1}-B M O$ duality $[51,50]$. In the standard way this gives uniform-in-time control of the vortex stretching term. The structural blow-up assumptions are provided to ensure uniform-in-time control of the $B M O$ norm of a multiplier (multiplied against the vortex stretching term). In the algebraic case, this is enabled by the logarithm's depletive effect on the unboundedness of the mean oscillations of polynomial functions, an effectiveness witnessed by the remarkable fact (cf. a proof by Stein in [102]) that there exists a constant $C(d, n)$ so that, for any polynomial on $\mathbb{R}^{n}$ of degree less than or equal to $d$,

$$
\|\log |P|\|_{B M O} \leq C(d, n) \text {. }
$$

Significantly, the constant is independent of the coefficients and this allows us to introduce time-dependent algebraic comparability conditions 
on the spatial profiles prior to a possibly singular time in a manner that preserves time-independent estimates on the $B M O$ norms of the logarithms of these profiles.

The second strategy is contained in Section 4.4. There we are interested in a very mild, purely geometric assumption that leads to a uniform-in-time $L \log L$ bound on the vorticity, effectively breaking the aforementioned scaling. More precisely, the assumption is a uniformin-time boundedness of the localized vorticity direction in a weighted

local version of the space of bounded mean oscillations, $\widetilde{b m o} \frac{1}{|\log r|}$. An interesting feature of this space (cf. [71]) is that it allows for discontinuous functions exhibiting very violent singularities, e.g. those along the lines of $\sin \log \mid \log$ ( something algebraic $) \mid$. Thus, the vorticity direction can blow-up in a geometrically spectacular fashion - every point on the unit sphere being a limit point - and the $L \log L$ bound will still hold.

\subsection{Preliminaries from harmonic analysis}

Here we review needed results from harmonic analysis and present a lemma which will connect these ideas to the PDE context of Section 4.3.2. Following [103], the maximal function of a distribution $f$ is defined for all $x \in \mathbb{R}^{n}$ as,

$$
M_{h} f(x)=\sup _{t>0}\left|f * h_{t}(x)\right|
$$

where $h$ is a fixed test function supported on the unit ball so that $\int h d x=1$ and $h_{t}$ denotes $t^{-n} h(x / t)$.

Definition 4.2.1. The distribution $f$ is in the Hardy space $\mathcal{H}^{1}$ if

$$
\|f\|_{\mathcal{H}^{1}}:=\left\|M_{h} f\right\|_{1}<\infty .
$$

In [29], Coifman, Lions, Meyer, and Semmes reformulated and refined some key features of the 'sequential' theory of compensated compactness within the framework of Hardy spaces, the key idea being that certain nonlinear quantities exhibit cancellations which lead to greater regularity. One such result is the Div-Curl lemma.

Lemma 4.2.2. (Coifman, Lions, Meyer, Semmes - [29]) If E, $B \in$ $L^{2}\left(\mathbb{R}^{3}\right)^{3}$ with $\nabla \cdot E=0$ and $\nabla \times B=0$ (in the sense of distributions), 
then $E \cdot B \in \mathcal{H}^{1}$, and there exists a universal constant $C$ such that,

$$
\|E \cdot B\|_{\mathcal{H}^{1}} \leq C\|E\|_{L^{2}}\|B\|_{L^{2}} .
$$

Because weak solutions to the Navier-Stokes equations are divergence free and because the curl of a gradient is always equal to the trivial distribution, it follows that the advective term, $(u \cdot \nabla) u$, in the velocity-pressure formulation exhibits div-curl structure, as does the vortex stretching term, $(\omega \cdot \nabla) u$, in the vorticity-velocity formulation. Consequently, using Hardy spaces, refined regularity results can be established for weak solutions of 3D NSE (see Chapter 3.2 of [81] for a collection of such results).

The well known result of Fefferman (cf. $[50,51]$ as well as Stein's monograph [103]) establishes that the dual space of $\mathcal{H}^{1}$ is precisely the space of functions of bounded mean oscillation, $B M O$. By $f_{B}$ we denote the average of the locally integrable function $f$ over the ball $B$ of volume $|B|$; i.e., $f_{B}=|B|^{-1} \int_{B} f d x$. The mean oscillation of $f$ over $B$ is $|B|^{-1} \int\left|f-f_{B}\right| d x$ and this is the quantity that characterizes the space $B M O$.

Definition 4.2.3. The locally integrable function $f$ is in $B M O$ if $\|f\|_{B M O}<\infty$ where,

$$
\|f\|_{B M O}:=\sup _{x \in \mathbb{R}^{3} ; 0<r} \frac{1}{|B(x, r)|} \int_{B(x, r)}\left|f(y)-f_{B(x, r)}\right| d y .
$$

Several results about the space $B M O$ will expedite future work. An elementary fact is that, if $g \in B M O$, then the following implication holds,

$$
\begin{gathered}
g(x)+m \leq f(x) \leq g(x)+M \text { for a. e. } x \in \mathbb{R}^{3} \\
\Longrightarrow \quad\|f\|_{B M O} \leq\|g\|_{B M O}+M-m .
\end{gathered}
$$

The above is easy to see by directly comparing $\left|f-f_{B}\right|$ to $\mid g+M-$ $(g+m)_{B} \mid$ and concluding that, for all balls $B$, we have,

$$
\frac{1}{|B|} \int_{B}\left|f-f_{B}\right| d x \leq \frac{1}{|B|} \int_{B}\left|g-g_{B}\right| d x+M-m .
$$

We include a technical lemma regarding certain point-wise multipliers on $B M O$ which appears in a paper by Iwaniec and Verde (cf. Lemma 
2.1 of [70]; see also Lemma 5.10 of [23]). This result will allow us to use the standard $\mathcal{H}^{1}-B M O$ duality in an essentially local context instead of working in the local versions of these spaces. It is worth mentioning that our results can be achieved in the local spaces using a local non-homogeneous version of the Div-Curl lemma (cf. [29]) paired with various local $h^{1}-b m o$ dualities (cf. [23]) thereby obtaining estimates on the localized vortex stretching term.

Lemma 4.2.4. (Iwaniec and Verde - [70]) Suppose $h \in B M O\left(\mathbb{R}^{n}\right)$ and $\phi \in C_{0}^{1}\left(\mathbb{R}^{n}\right)$ is supported on the ball $B$. Then $\phi h \in B M O\left(\mathbb{R}^{n}\right)$ and we have,

$$
\|\phi h\|_{B M O} \leq C(n, R)\|\nabla \phi\|_{\infty}\left(\left(\|h\|_{B M O}+\left|\frac{1}{|B|} \int_{B} h d x\right|\right) .\right.
$$

The space $B M O$ enjoys an intimate connection with the logarithm. This is illustrated in the following lemma due to Stein.

Lemma 4.2.5. (Stein - [102]) Let $P$ be any polynomial in $\mathbb{R}^{n}$ of degree less than or equal to $d$. Then there exists a constant $C=C(d, n)$ so that $\log |P| \in B M O$ and, moreover,

$$
\|\log |P|\|_{B M O} \leq C(d, n) .
$$

It turns out that the constant appearing above worsens as $d$ increases but does so linearly in $d$, a fact we will return to in Section 4.3 .

A variety of local and weighted versions of $\mathcal{H}^{1}$ and $B M O$ have been studied and will provide a context for the main result of Section 4.4. A local version of $B M O$, usually denoted by $b m o$, is defined by finiteness of the following expression,

$$
\|f\|_{b m o}=\sup _{x \in \mathbb{R}^{3}, 0<r<\delta} \Omega(f, I(x, r))+\sup _{x \in \mathbb{R}^{3}, r \geq \delta} \frac{1}{|I(x, r)|} \int_{I(x, r)}|f(y)| d y,
$$

for some positive $\delta$.

If $f \in L^{1}$, we can focus on small scales, e.g., $0<r<\frac{1}{2}$. Let $\phi$ be a positive, non-decreasing function on $\left(0, \frac{1}{2}\right)$. We define local weighted spaces of bounded mean oscillations as in [83], i.e.,

$$
\|f\|_{\widetilde{b m o}_{\phi}}=\|f\|_{L^{1}}+\sup _{x \in \mathbb{R}^{3}, 0<r<\frac{1}{2}} \frac{\Omega(f, I(x, r))}{\phi(r)} .
$$


Of special interest will be the spaces $\widetilde{b m o}=\widetilde{b m o_{1}}$ and $\widetilde{b m o} \frac{1}{|\log r|}$. Generally speaking these local, weighted spaces of bounded mean oscillation are convenient when working with pointwise multipliers (cf. [71, 87, 83]). This is illustrated in a useful theorem from [83] which we include as a lemma.

Lemma 4.2.6. Let $h$ be in $\widetilde{b m o}$ and $g$ in $L^{\infty} \cap \widetilde{b_{m o}} \frac{1}{|\log r|}$. Then,

$$
\|g h\|_{\widehat{b m o}} \leq c(n)\left(\|g\|_{\infty}+\|g\|_{\widehat{b m o} \frac{1}{\sqrt{\log r \mid}}}\right)\|h\|_{\widehat{b m o}} .
$$

Essentially, then, the space of pointwise $\widetilde{b m o}$ multipliers coincides with $L^{\infty} \cap \widehat{b m o} \frac{1}{|\log r|}$.

We conclude this section by recalling Coifman and Rochberg's estimate on $\|\log M f\|_{B M O}$ (cf. [30]). Let $M$ denote the Hardy-Littlewood maximal operator. Coifman and Rochberg [30] obtained a characterization of $B M O$ in terms of images of the logarithm of the maximal function of non-negative locally integrable functions (plus a bounded part). The main ingredient in demonstrating one direction is the following estimate,

$$
\|\log M f\|_{B M O} \leq c(n)
$$

for any locally integrable function $f$. Note that this bound is completely independent of $f$. Moreover, this estimate remains valid if we replace $M f$ with $\mathcal{M} f=(M \sqrt{|f|})^{2}$ (cf. [70]); the advantage of working with $\mathcal{M}$ is that the $L^{2}$-maximal theorem implies that,

$$
\|\mathcal{M} f\|_{1} \leq c(n)\|f\|_{1},
$$

a bound that does not hold for the standard maximal operator $M$.

\subsection{Blow-up scenarios exhibiting logarith- mically subcritical anisotropic diffu- sion}

\subsubsection{Amenable blow-up rates}

This subsection is dedicated to describing amenable blow-up rates and proving a lemma which will connect these structures to the PDE context of Section4.3.2. We denote by $\log ^{m}(x)$ an iterated composition 
of the logarithm with itself $m-1$ times, that is, $\log ^{m}(x)=\log \circ \cdots \circ$ $\log \left(\sqrt{e_{m}+x^{2}}\right)$ where $m$ applications the natural logarithm are carried out and $e_{m}$ is a power of $e$ defined so that $\log ^{m}(0)=0$. We will use the following notation when defining functions of $(x, t)$ possessing singularities at time $T$ in the spatial set $S \subset \mathbb{R}^{3}$,

$$
Q_{T}\left(x_{0}, R, S\right)=B\left(x_{0}, R\right) \times(0, T) \cup\left(\left(B\left(x_{0}, R\right) \backslash S\right) \times\{T\},\right.
$$

and,

$$
\Omega_{T}(S)=\mathbb{R}^{3} \times(0, T) \cup\left(\mathbb{R}^{3} \backslash S\right) \times\{T\},
$$

where $R>0$ and $x_{0} \in \mathbb{R}^{3}$. In contexts where $x_{0}$ and $R$ are fixed we make the abbreviation $Q_{T}(S)=Q_{T}\left(x_{0}, R, S\right)$.

Definition 4.3.1. Fix $x_{0} \in \mathbb{R}^{3}, R, T>0, m \in \mathbb{N}_{0}$ and a set of measure zero, $S$, which is contained in a compact subset of $B\left(x_{0}, R\right)$.

a. An amenable blow-up rate of order $m$ on $Q_{T}(S)$ is a function, $D(x, t): \Omega_{T}(S) \rightarrow[0, \infty)$, which additionally satisfies,

i. there exists $M_{0}>0$ so that $\sup _{0<t \leq T}\left\|\log ^{m+1} D(x, t)\right\|_{B M O}<$ $M_{0}$,

ii. there exist $M_{1}, M_{2}>0$ so that $1 / M_{1} \leq D(x, t)$ on $Q_{T}(S)$ and $D(x, t) \leq M_{2}$ on $B\left(x_{0}, R\right)^{c} \times(0, T]$.

b. A function $f(x, t): Q_{T}(S) \rightarrow \mathbb{R}$ exhibits an amenable blow-up rate of order 0 on $Q_{T}(S)$ if there exists an amenable blow-up rate of order $0, D(x, t)$, on $Q_{T}(S)$, so that, for some $C_{*}>1$,

$$
D(x, t) \leq|f(x, t)| \leq C_{*} D(x, t) \quad \text { for all }(x, t) \in Q_{T}(S) .
$$

c. A function $f(x, t): Q_{T}(S) \rightarrow \mathbb{R}$ exhibits an amenable blow-up rate of order 1 on $Q_{T}(S)$ if there exists an amenable blow-up rate of order $1, D(x, t)$, on $Q_{T}(S)$, so that, for some $C_{*}>0, A \geq 1$, and a scalar function $\alpha:[0, T] \rightarrow[1, A]$,

$$
D(x, t) \leq|f(x, t)| \leq C_{*} D(x, t)^{\alpha(t)} \quad \text { for all }(x, t) \in Q_{T}(S) .
$$

The condition (4.3.1.a.i) will prove crucial in establishing our estimates in Section 4.3.2. At face value, however, it is not clearly motivated. To address this we specify an expansive class of functions 
which are simultaneously amenable blow-up rates of order 0 and are reasonable blow-up scenarios given what is known about the structure of possible singularities in weak solutions of 3D NSE. In the following, $\mathbb{R}\left[x_{1}, x_{2}, x_{3}\right]$ denotes the ring of tri-variate polynomials with coefficients in $\mathbb{R}$.

Definition 4.3.2. Use the notations of Definition 4.3.1 and fix $d \in \mathbb{N}$. The function $D(x, t): \Omega_{T}(S) \rightarrow[0, \infty)$ is an algebraic blow-up rate of degree $d$ on $Q_{T}(S)$ if there exist functions $\tau:(0, T] \rightarrow[0, \infty), \alpha$ : $(0, T] \rightarrow(0, \infty)$, and $\rho(x, \cdot):(0, T] \rightarrow \mathbb{R}\left[x_{1}, x_{2}, x_{3}\right]$ so that,

$$
D(x, t)=\left(\frac{1}{|\rho(x, t)|+\tau(t)}\right)^{\alpha(t)} \text { on } Q_{T}(S),
$$

and we additionally have,

i. for all $t \in(0, T], \rho(x, t)$ is a polynomial of degree less than or equal to $d$ and the zeros of $\rho(x, T)$ are contained in $S$,

ii. there exists $A \geq 1$ so that $\alpha$ takes values in $\left[A^{-1}, A\right]$,

iii. $\tau(t)>0$ for $t \in(0, T)$ and vanishes as $t$ approaches $T$,

iv. the condition (4.3.1.a.ii.) is satisfied.

Remark 4.3.1. We have presented these algebraic blow-up rates because they constitute a concrete class of amenable blow-up rates of order 0. To verify this, we check that condition (4.3.1.a.i.) is satisfied, which is clear if we first expand $\log D(x, t)$ as,

$$
\log (D(x, t))=-\alpha(t) \log (|\rho(x, t)|+\tau(t)),
$$

and then observe that,

$$
\begin{aligned}
& \max \{\log |\rho(x, t)|, \log \tau(t)\} \leq \log (|\rho(x, t)|+\tau(t)) \\
& \leq \log (2)+\max \{\log |\rho(x, t)|, \log \tau(t)\} .
\end{aligned}
$$

Recalling the fact that $B M O$ is a lattice, i.e. if $f, g \in B M O$, then,

$$
\|\max \{f, g\}\|_{B M O} \leq 2\left(\|f\|_{B M O}+\|g\|_{B M O}\right),
$$

we are able to conclude by applying the implication (4.2.1) in conjunction with Lemma 4.2.5. 
Our main lemma contextualizes amenable blow-up rates to their application in Section 4.3.2. As presently defined, the blow-up rates require that $f(x, t)$ (as given in Definition 4.3.1) is bounded away from zero. To accommodate functions possibly not bounded away from zero we employ an auxiliary function, $F=\sqrt{e_{m}+f^{2}}$, where $e_{m}$ is determined by the order of the amenable blow-up rate in question. Based on the sign of $f$ we define,

$F_{+}=\left\{\begin{array}{ll}F, & \text { if } f \text { is non-negative, } \\ 1, & \text { otherwise }\end{array} \quad F_{-}= \begin{cases}1, & \text { if } f \text { is non-negative } \\ F, & \text { otherwise }\end{cases}\right.$

The factorization $F^{\operatorname{sign} f}=F_{+}\left(F_{-}\right)^{-1}$ will allow us to independently impose blow-up assumptions on a function's positive and negative components.

Lemma 4.3.3. Fix $R_{0}>0$. Let $S^{\prime} \subset \mathbb{R}^{3}$ be a set of Lebesque measure zero for which $S^{\prime} \cap B\left(0, R_{0}\right) \subset B\left(0, R_{0}^{\prime}\right)$ where $0<R_{0}^{\prime}<R_{0}$. Suppose the function $f: \Omega_{T}\left(S^{\prime}\right) \rightarrow \mathbb{R}$ satisfies $f \in L^{\infty}\left((0, T] ; L^{1}\left(B\left(0, R_{0}\right)\right)\right)$. Fix $\phi \in C_{0}^{1}\left(\mathbb{R}^{3}\right)$ so that $\phi$ is supported on $B(0, R)$ and let $S$ be a set of measure zero satisfying $S^{\prime} \cap B\left(0, R_{0}\right) \subset S \subset B\left(0, R_{0}\right)$.

a. If $\left.f\right|_{Q_{T}(S)}$ exhibits an amenable blow-up of order 0 in $Q_{T}(S)$, then,

$$
\sup _{0<t \leq T}\|\phi(x) \log |f(x, t)|\|_{B M O}<\infty
$$

b. If $\left.F_{+}\right|_{Q_{T}(S)}$ and $\left.F_{-}\right|_{Q_{T}(S)}$ exhibit amenable blow-up rates of order 0 in $Q_{T}(S)$, then,

$$
\sup _{0<t \leq T}\left\|\phi(x) \frac{f(x, t)}{F(x, t)} \log F(x, t)\right\|_{B M O}<\infty .
$$

c. If $\left.F_{+}\right|_{Q_{T}(S)}$ and $\left.F_{-}\right|_{Q_{T}(S)}$ exhibit amenable blow-up rates of order 1 in $Q_{T}(S)$, then,

$$
\sup _{0<t \leq T}\left\|\phi(x) \frac{f(x, t)}{F(x, t)} \log \log F(x, t)\right\|_{B M O}<\infty .
$$


Proof. (Part a.) Let $D(x, t)$ be the amenable blow-up rate exhibited by $\left.f\right|_{Q_{T}(S)}$. We will freely reference the constants associated with $D$ in the statement of Definition 4.3.1 and subsequently make the abbreviations $Q_{T}(S)=Q_{T}\left(0, R_{0}, S\right)$ and $B=B\left(0, R_{0}\right)$. Define an extension, $\widetilde{F}$, of $\left.f\right|_{Q_{T}(S)}$ to $\Omega_{T}(S)$ by $\widetilde{F}=|f|$ on $Q_{T}(S)$ and $\widetilde{F}=1$ on $B^{c} \times(0, T]$. Set $\widetilde{C}_{*}=\max \left\{C_{*}, M_{2}\right\}$ and $\widetilde{D}(x, t)=\max \left\{D(x, t), M_{1}^{-1}\right\}$. These definitions ensure that, for all $(x, t) \in \Omega_{T}(S)$,

$$
\frac{1}{\widetilde{C}_{*}} \widetilde{D}(x, t) \leq \widetilde{F}(x, t) \leq \widetilde{C}_{*} \widetilde{D}(x, t),
$$

and, taking logarithms,

$$
\log (\widetilde{D}(x, t))-\log \widetilde{C}_{*} \leq \log (\widetilde{F}(x, t)) \leq \log (\widetilde{D}(x, t))+\log \widetilde{C}_{*} .
$$

Recalling the implication (4.2.1), we see that,

$$
\|\log \widetilde{F}(x, t)\|_{B M O} \leq C\left(M_{0}, \tilde{C}_{*}\right) .
$$

Because $|f|=\widetilde{F}$ on the support of $\phi$ we have,

$$
\begin{aligned}
\|\phi \log |f|\|_{B M O} & =\|\phi \log \widetilde{F}\|_{B M O} \\
& \leq C\|\nabla \phi\|_{\infty}\left(\|\log \widetilde{F}\|_{B M O}+\left|\frac{1}{|B|} \int_{B} \log \widetilde{F} d x\right|\right),
\end{aligned}
$$

where we have applied Lemma 4.2.4. The average appearing above is uniformly bounded in time; this follows from the facts that $M_{1}^{-1} \leq|f|$ and $f \in L^{\infty}\left((0, T] ; L^{1}\left(B\left(0, R_{0}\right)\right)\right)$.

(Part b.) We begin by remarking that, for all $y \in \mathbb{R}$,

$$
\begin{aligned}
\operatorname{sign} y \log \left(\sqrt{1+y^{2}}\right)-1 & \leq \frac{y}{\sqrt{1+y^{2}}} \log \left(\sqrt{1+y^{2}}\right) \\
& \left.\leq \operatorname{sign} y \log \sqrt{1+y^{2}}\right)+1 .
\end{aligned}
$$

Taking $y=|f(x, t)|$, in light of implication (4.2.1) it is sufficient to work with the function $(\operatorname{sign} f) \log F$, where $F, F_{+}$, and $F_{-}$are defined as in the comments preceding the statement of the lemma. Those definitions were motivated by the fact that $F^{\operatorname{sign} f}=F_{+}\left(F_{-}\right)^{-1}$ and, so,

$$
\log \left(F^{\operatorname{sign} f}\right)=\log \left(F_{+}\right)-\log \left(F_{-}\right) .
$$


Let $G$ be the extension by 1 of $\left.\left(f F^{-1} \log (F)\right)\right|_{Q_{T}(S)}$ from $Q_{T}(S)$ to $\Omega_{T}(S)$ and adopt the notation from the proof of part a. of this lemma for definitions of functions analogous to those mentioned above where we designate by $D_{ \pm}$the blow-up rates exhibited by $F_{ \pm}$. Then, on $\Omega_{T}(S)$, we have,

$$
\log \left(\widetilde{F}_{+}\right)-\log \left(\widetilde{F}_{-}\right)-1 \leq G \leq \log \left(\widetilde{F}_{+}\right)-\log \left(\widetilde{F}_{-}\right)+1
$$

and, therefore,

$$
\|G\|_{B M O} \leq\left\|\log \left(\widetilde{F}_{+}\right)\right\|_{B M O}+\left\|\log \left(\widetilde{F}_{-}\right)\right\|_{B M O}+2
$$

Appealing to part a. of the lemma, the dominating quantity is itself bounded uniformly in time. Extending this to the desired estimate proceeds directly,

$$
\left\|\phi \frac{f}{F} \log (F)\right\|_{B M O} \leq C\|\nabla \phi\|_{\infty}\left(\|G\|_{B M O}+\left|\frac{1}{B} \int_{B} \log \widetilde{G} d x\right|\right),
$$

which, again, is finite by assumptions on $f$.

(Part c.) We begin similarly, noting,

$$
(\operatorname{sign} f) \log \log F-1 \leq \frac{f}{F} \log \log F \leq(\operatorname{sign} f) \log \log F+1 .
$$

This leads us to consider,

$$
\log \left((\log F)^{\operatorname{sign} f}\right)=\log \left((\log F)_{+}\right)+\log \left((\log F)_{-}\right)
$$

where,

$$
(\log F)_{+}=\max \left\{(\log F)^{\operatorname{sign} f}, 1\right\}
$$

and,

$$
(\log F)_{-}=\min \left\{(\log F)^{\operatorname{sign} f}, 1\right\} .
$$

It is easy to check that $(\log F)_{ \pm}=\log \left(F_{ \pm}\right)$, and, therefore,

$$
\frac{f}{F} \log \log F=\log \log \left(F_{+}\right)-\log \log \left(F_{-}\right) .
$$


A further observation is that, taking $D_{ \pm}(x, t)$ to be the amenable blowup rates exhibited by $F_{ \pm}$, and setting $k_{ \pm}^{*}=\log \left(A_{ \pm} M_{1, \pm}+\log C_{*, \pm}\right)$ (these are constants associated with $D_{ \pm}$as in Definition 4.3.1.a.), we have for $(x, t) \in Q_{T}$,

$$
\log \log \left(C_{*} D_{ \pm}(x, t)^{\alpha_{ \pm}(t)}\right) \leq \log \log \left(D_{ \pm}(x, t)\right)+k_{ \pm}^{*},
$$

and, recalling the comparability condition from Definition 4.3.1.c., this implies that,

$$
\log \log \left(D_{ \pm}(x, t)\right) \leq F_{ \pm} \leq \log \log \left(D_{ \pm}(x, t)\right)+k_{ \pm}^{*} .
$$

The conclusion now follows in the same fashion laid out in part b.; we omit the details.

\subsubsection{Main result}

Weak solutions to the 3D incompressible Navier-Stokes equations are functions which satisfy (distributionally) the following system of PDEs,

$$
\left\{\begin{array}{l}
\partial_{t} u+(u \cdot \nabla) u=-\nabla p+\nu \Delta u, \\
\nabla \cdot u=0 ; \quad u(x, 0)=u_{0} \in H,
\end{array}\right.
$$

where $H$ is the $L^{2}$ closure of the divergence free test functions and the initial datum is understood in the sense of weak continuity (cf. [35] for details). The evolution of the vorticity, $\omega=\nabla \times u$, is of special interest to us and satisfies,

$$
\left\{\begin{array}{c}
\partial_{t} \omega+(u \cdot \nabla) \omega=(\omega \cdot \nabla)+\nu \Delta \omega, \\
\nabla \cdot \omega=0 .
\end{array}\right.
$$

For simplicity, we consider a weak solution on $\mathbb{R}^{3} \times(0, \infty)$, evolving from the initial data $u_{0}$. We also require that the initial vorticity, $\omega_{0}=\nabla \times u_{0}$, is in $L^{1} \cap L^{2}$.

The standard regularity results for weak solutions (cf. [35]) consist of the a priori bounds,

$$
\sup _{0<t<T^{\prime}}\|u\|_{L^{2}\left(\mathbb{R}^{3}\right)}^{2}<\infty \text { and } \int_{0}^{T^{\prime}}\|\nabla u\|_{L^{2}\left(\mathbb{R}^{3}\right)}^{2} d t<\infty,
$$


for any $T^{\prime}>0$. In addition, since $\omega_{0} \in L^{1}$, a result from [31] ensures that,

$$
\sup _{0 \leq t \leq T^{\prime}}\|\omega\|_{L^{1}\left(\mathbb{R}^{3}\right)}<\infty .
$$

Note that since $\omega_{0} \in L^{2}$, our weak solution locally-in-time coincides with the smooth solution; let $T$ be the first (possible) singular time.

Fix a 'macro-scale,' $R_{0}>0$, with the property that the intersection of $B\left(0, R_{0}\right) \times\{T\}$ with the singular set at time $T$ is nonempty. Fix $0<\epsilon<R_{0}$. Our estimates are intended for integrals over the spatial set $B\left(0, R_{0}-\epsilon\right)$ and localization is achieved via multiplying equation (4.3.3) by a smooth cut-off function $\psi$ taking values in $[0,1]$ and satisfying,

$$
\operatorname{supp} \psi \subset B\left(0, R_{0}\right), \psi=1 \text { on } B\left(0, R_{0}-\epsilon\right), \text { and } \frac{|\nabla \psi|}{\psi^{\rho}} \leq \frac{c}{\epsilon},
$$

where $\rho \in(0,1)$ is fixed. Instead of studying the evolution of $|\omega|$ and $\left|\omega_{k}\right|$ directly we introduce an auxiliary function. This approach is an adaptation of that taken by Constantin in [31]. Define $q(y)=$ $\sqrt{1+y^{2}}: \mathbb{R} \rightarrow \mathbb{R}$ and let $w_{k}=q\left(\omega_{k}\right): \mathbb{R}^{3} \rightarrow \mathbb{R}$. From these definitions it is immediate that,

$$
\left|\omega_{k}\right| \leq w_{k}, \quad-1 \leq q^{\prime}\left(\omega_{k}\right)=\frac{\omega_{k}}{w_{k}} \leq 1, \text { and } 0<q^{\prime \prime}\left(\omega_{k}\right)=\frac{1}{w_{k}^{3}} \leq 1 .
$$

For convenience we recall the following elementary facts (for $y \geq 1$ ),

$$
0 \leq-\log ^{\prime \prime}(y) \leq \log ^{\prime}(y) \leq 1, y \log ^{\prime} y=1 \text {, and } 0=\log ^{\prime}(y)+y \log ^{\prime \prime}(y) \text {. }
$$

To allow $\omega_{k}$ to have varying sign we define the functions $w_{k,+}$ and $w_{k,-}$ in the same manner as (4.3.1). Recall that $\Lambda_{t}(y)=\{x:|\omega(x, t)| \geq y\}$ and note that throughout the remainder of this paper $c_{1}$ denotes a fixed constant taken to be greater than one. The role of $c_{1}$ lies in specifying the threshold for the super-level sets $\Lambda_{t}(y)$ as fractions of the supremum norm of the modulus of the vorticity at the time $t$, i.e. $y=c_{1}^{-1}\|\omega(\cdot, t)\|_{\infty}$.

We include two theorems, one each for the case of solutions possessing vorticity components which exhibit amenable blow-up rates of order 0 and of order 1 . The proof of the order 0 case extends easily to the case of blow-ups of order 1 and the proof of the second result is accordingly terse. 
Theorem 4.3.4. Let $u$ be a Leray solution to $(4.3 .2)$ on $\mathbb{R}^{3} \times(0, \infty)$, and suppose additionally that $\omega_{0}=\nabla \times u_{0} \in L^{1} \cap L^{2}$. Denote by $T$ the first singular time and by $S$ the singular set of $\omega$ at time $T$. Fix positive values $R_{0}$ and $\epsilon$ so that $\emptyset \neq S \cap B\left(0, R_{0}\right) \subset B\left(0, R_{0}-\epsilon\right)$ - i.e. there are singular points in $B\left(0, R_{0}\right)$ but all such points are in $B\left(0, R_{0}-\epsilon\right)$ - and let $Q_{T}(S)=Q_{T}\left(0, R_{0}, S\right)$.

(i) If $\left.w_{k,+}\right|_{Q_{T}(S)}$ and $\left.w_{k,-}\right|_{Q_{T}(S)}$ each exhibit amenable blow-up rates of order 0 in $Q_{T}(S)$, then there exists a positive value $M_{k}$ for which,

$$
\sup _{t \in[0, T]} \int_{B\left(0, R_{0}-\epsilon\right)}\left|\omega_{k}(x, t)\right| \log \left(\sqrt{1+\left|\omega_{k}(x, t)\right|^{2}}\right) d x<M_{k} .
$$

(ii) Let $c_{1}$ ba a fixed constant which is greater than 1. If, for all $k$, the premises of part (i) are satisfied, then there exists a positive constant $M_{0}$ so that, for $t \in[0, T]$,

$$
\begin{aligned}
& \operatorname{Vol}\left(\Lambda_{t}\left(\frac{1}{c_{1}}\|\omega(t)\|_{L^{\infty}\left(B\left(0, R_{0}-\epsilon\right)\right)}\right)\right) \\
& \leq \frac{M_{0}}{\|\omega(t)\|_{L^{\infty}\left(B\left(0, R_{0}-\epsilon\right)\right)} \log \left(1+\|\omega(t)\|_{L^{\infty}\left(B\left(0, R_{0}-\epsilon\right)\right)}\right)} .
\end{aligned}
$$

Theorem 4.3.4 applies to any local, spatially-algebraic blow-up scenario in which the degrees of the polynomials stay uniformly bounded near the (possible) singular time. Geometrically, this corresponds to the number of vortex filaments being uniformly bounded as the flow approaches the singular time. This is somewhat unsatisfactory since, $a$ priori, one cannot rule out a scenario in which the number of coherent structures runs off to infinity. The technical reason behind this restriction is that the bound on $\|\log |P|\|_{B M O}$ blows up as the degree of $P$, $d$, goes to infinity. Fortunately, the bound on $\|\log |P|\|_{B M O}$ is linear in $d$ (cf. [49]). Consequently, although the bound on the distribution function, i.e. on the total volume of the super-level sets, will blow up with $d$, at least in the case of comparable volumes, the bound on the volume of a single vortex filament will still be subcritical (which suffices due to the local nature of the argument).

As will be seen, our energy inequality-type method depends heavily on classical techniques which leads us to consider local-in-time smooth 
solutions. It is possible to extend the estimates to some weak solutions by considering a sequence of smooth approximations, e.g. the retarded mollifiers from [20], but, as our estimates hinge on assumptions beyond the initial data, $u_{0}$, we would have to ensure these are met by a convergent subsequence of approximate solutions.

Proof. The evolution of $\psi w_{k} \log \left(w_{k}\right)$ can be established from the evolution of $\omega_{k}$ by first writing,

$$
\begin{aligned}
& \partial_{t}\left(\psi w_{k} \log w_{k}\right)=\psi q^{\prime}\left(\omega_{k}\right)\left(\log w_{k}+w_{k} \log ^{\prime} w_{k}\right)\left(\partial_{t} \omega_{k}\right) \\
& =\psi q^{\prime}\left(\omega_{k}\right)\left(\log w_{k}+w_{k} \log ^{\prime} w_{k}\right)\left(\nu \Delta \omega_{k}-(u \cdot \nabla) \omega_{k}+(\omega \cdot \nabla) u_{k}\right),
\end{aligned}
$$

which leads to (noting tacit summation over terms involving indices other than $k$ ),

$$
\begin{aligned}
\partial_{t} & \left(\psi w_{k} \log w_{k}\right)-\nu \psi \Delta w_{k}\left(\log w_{k}+w_{k} \log ^{\prime} w_{k}\right) \\
& +\nu \psi q^{\prime \prime}\left(\omega_{k}\right)\left(\partial_{i} \omega_{k}\right)^{2}\left(\log w_{k}+w_{k} \log ^{\prime} w_{k}\right) \\
= & \psi(\omega \cdot \nabla) u_{k} q^{\prime}\left(\omega_{k}\right)\left(\log w_{k}+w_{k} \log ^{\prime} w_{k}\right) \\
& -\psi(u \cdot \nabla) w_{k}\left(\log w_{k}+w_{k} \log ^{\prime} w_{k}\right)
\end{aligned}
$$

By integrating in space and time and dropping the positive quantity involving $q^{\prime \prime}$ from the left hand side, we obtain the following energy inequality-type estimate,

$$
\begin{aligned}
& \int \psi(t) w_{k}(t) \log w_{k}(t) d x-\nu \int_{0}^{t} \int \psi \Delta w_{k}\left(\log w_{k}+w_{k} \log ^{\prime} w_{k}\right) d x d s \\
& \leq \int_{0}^{t} \int \psi(\omega \cdot \nabla) u_{k} q^{\prime}\left(\omega_{k}\right)\left(\log w_{k}+w_{k} \log ^{\prime} w_{k}\right) d x d s \\
& \quad-\int_{0}^{t} \int \psi(u \cdot \nabla) w_{k}\left(\log w_{k}+w_{k} \log ^{\prime} w_{k}\right) d x d s \\
& \quad+\int \psi w_{k, 0} \log w_{k, 0} d x .
\end{aligned}
$$

The properties in (4.3.5) enable several key cancellations. For the dis- 
sipative terms, integration by parts reveals that,

$$
\begin{aligned}
& \int_{0}^{t} \int\left(\partial_{j}^{2} w_{k}\right) \psi \log w_{k} d x d s \\
& =\int_{0}^{t} \int\left(w_{k}\left(\partial_{j}^{2} \psi\right) \log w_{k}+w_{k}\left(\partial_{j} \psi\right)\left(\partial_{j} w_{k}\right) \log ^{\prime} w_{k}\right) d x d s \\
& \quad-\int_{0}^{t} \int\left(\partial_{j} w_{k}\right)^{2} \psi \log ^{\prime} w_{k} d x d s
\end{aligned}
$$

and,

$$
\begin{aligned}
& \int_{0}^{t} \int\left(\partial_{j}^{2} w_{k}\right) \psi w_{k} \log ^{\prime} w_{k} d x d s \\
& =-\int_{0}^{t} \int w_{k}\left(\partial_{j} \psi\right)\left(\partial_{j} w_{k}\right) \log ^{\prime} w_{k} d x d s \\
& \quad-\int_{0}^{t} \int\left(\partial_{j} w_{k}\right)^{2} \psi\left(\log ^{\prime} w_{k}+w_{k} \log ^{\prime \prime} w_{k}\right) d x d s .
\end{aligned}
$$

A cancellation occurs upon adding the above equations leaving us with,

$$
\begin{aligned}
& -\nu \int_{0}^{t} \int\left(\partial_{j}^{2} w_{k}\right) \psi\left(\log w_{k}+w_{k} \log ^{\prime} w_{k}\right) d x d s \\
& =\nu \int_{0}^{t} \int\left(\partial_{j} w_{k}\right)^{2} \psi\left(2 \log ^{\prime} w_{k}+w_{k} \log ^{\prime \prime} w_{k}\right) d x d s \\
& \quad-\nu \int_{0}^{t} \int w_{k}\left(\partial_{j}^{2} \psi\right) \log w_{k} d x d s .
\end{aligned}
$$

Again noting (4.3.5), the integrand of the first term is positive and, therefore, is dropped from the left hand side of estimate (4.3.6). The second term can be dominated by an a priori finite quantity arising from the standard energy inequality for weak solutions in conjunction with the fact that our spatial integral is over a set of finite measure. More precisely,

$$
\begin{aligned}
\int_{0}^{t} \int w_{k}\left(\partial_{j}^{2} \psi\right) \log w_{k} d x d s & \leq C \int_{0}^{T} \int_{B\left(0, R_{0}\right)} w_{k}^{2} d x d s \\
& =C \int_{0}^{T} \int_{B\left(0, R_{0}\right)}\left|\omega_{k}\right|^{2} d x d s+T\left|B\left(0, R_{0}\right)\right|
\end{aligned}
$$


The integrals arising from the transport term in (4.3.3) also enjoy substantial cancellations,

$$
\begin{aligned}
& \int_{0}^{t} \int \psi u \cdot \nabla w_{k}\left(\log w_{k}+w_{k} \log ^{\prime} w_{k}\right) d x d s \\
& =\int_{0}^{t} \int \psi u_{j}\left(\partial_{j} w_{k}\right) w_{k} \log ^{\prime} w_{k} d x d s-\int_{0}^{t} \int \psi u_{j} w_{k}\left(\partial_{j} w_{k}\right) \log ^{\prime} w_{k} d x d s \\
& \quad-\int_{0}^{t} \int u_{j} w_{k} \log w_{k} \partial_{j} \psi d x d s \\
& =-\int_{0}^{t} \int u_{j} w_{k} \log w_{k} \partial_{j} \psi d x d s .
\end{aligned}
$$

Noting that $\log (y) \leq 4 y^{1 / 4}$,

$$
\begin{aligned}
& \left|\int_{0}^{t} \int u_{j} w_{k} \log w_{k}\right| \partial_{j} \psi|d x d s| \\
& \leq C \int_{0}^{T} \int\left(\left|\partial_{j} \psi\right|^{1 / 4}\left|u_{j}\right|\right)\left(\left|\partial_{j} \psi\right|^{1 / 2}\left|w_{k}\right|\right)\left|w_{k} \partial_{j} \psi\right|^{1 / 4} d x d s \\
& \leq C \int_{0}^{T}\left\|\psi^{\rho / 4} u\right\|_{4}|| w_{k}\left\|_{L^{1}\left(B\left(0, R_{0}\right)\right)}^{1 / 4}|| w_{k}\right\|_{L^{2}\left(B\left(0, R_{0}\right)\right)} \\
& \leq C \sup _{0<t \leq T}\left\|w_{k}\right\|_{L^{1}\left(B\left(0, R_{0}\right)\right)}^{1 / 4} \int_{0}^{T}\left\|\nabla\left(\psi^{\rho / 4} u\right)\right\|_{2}\left\|w_{k}\right\|_{L^{2}\left(B\left(0, R_{0}\right)\right)},
\end{aligned}
$$

where we have used Hölder's inequality and the Sobolev inequality. From here and in light of (4.3.7), a commutator estimate on the gradient of the localized velocity allows the extension of the above estimate to one in terms of a priori finite quantities.

The integral on the last line of the right hand side of (4.3.6) is finite by our assumptions on the initial data.

At this point, based on (4.3.6), we have established,

$$
\begin{aligned}
& \int\left(\psi w_{k} \log w_{k}\right)(t) d x \\
& \leq\left|\int_{0}^{t} \int \psi \omega \cdot \nabla u_{k} q^{\prime}\left(\omega_{k}\right)\left(\log w_{k}+w_{k} \log ^{\prime} w_{k}\right) d x d s\right|+R,
\end{aligned}
$$

where $R$ is comprised of those a priori bounded quantities accumulated in the preceding estimates. Noting that,

$$
\psi q^{\prime}\left(\omega_{k}\right) w_{k} \log ^{\prime} w_{k} \leq 1
$$


an a priori bound follows for that part of the as of yet unbounded quantity leaving us with,

$$
\int\left(\psi w_{k} \log w_{k}\right)(t) d x \leq\left|\int_{0}^{t} \int \psi \omega \cdot \nabla u_{k} \frac{\omega_{k}}{w_{k}} \log w_{k} d x d s\right|+R .
$$

The $\mathcal{H}^{1}-B M O$ duality and the Div-Curl lemma lead to the estimate,

$$
\begin{aligned}
& \left|\int_{0}^{t} \int\left(\omega \cdot \nabla u_{k}\right)\left(\psi \frac{\omega_{k}}{w_{k}} \log w_{k}\right) d x d s\right| \\
& \leq \int_{0}^{T}\left\|\omega \cdot \nabla u_{k}\right\|_{\mathcal{H}^{1}}\left\|\psi \frac{\omega_{k}}{w_{k}} \log w_{k}\right\|_{B M O} d s \\
& \leq\left(\sup _{0<t \leq T}\left\|\psi \frac{\omega_{k}}{w_{k}} \log w_{k}\right\|_{B M O}\right) \int_{0}^{T}\left\|\omega \cdot \nabla u_{k}\right\|_{\mathcal{H}^{1}} d s \\
& \leq\left(\sup _{0<t \leq T}\left\|\psi \frac{\omega_{k}}{w_{k}} \log w_{k}\right\|_{B M O}\right)\left(\int_{0}^{T}\|\omega\|_{2}^{2} d t\right)^{1 / 2}\left(\int_{0}^{T}\|\nabla u\|_{2}^{2} d t\right)^{1 / 2} .
\end{aligned}
$$

By Lemma 4.3.3 and the standard regularity of Leray weak solutions all of the above are finite and we have thus established that, for all $0<$ $t \leq T,\left\|\psi w_{k} \log w_{k}(t)\right\|_{L^{1}\left(\mathbb{R}^{3}\right)}$ is majorized by time-independent a priori bounded quantities, the sum of which we label $M_{k}$. This completes our proof of part (i) of the theorem.

Part (ii) of the theorem is proven in two steps. For the first, let,

$$
\lambda(t)=\frac{1}{c_{1}}\|\omega(t)\|_{L^{\infty}\left(B\left(0, R_{0}-\epsilon\right)\right)},
$$

and observe that, for any $x \in B\left(0, R_{0}-\epsilon\right)$ where $|\omega(x, t)| \geq \lambda(t)$, direct computation affirms that,

$$
1 \leq \frac{c_{1}|\omega(x, t)|\left[\log \left(c_{1}\right)+\log (1+|\omega(x, t)|)\right]}{\|\omega(t)\|_{L^{\infty}\left(B\left(0, R_{0}-\epsilon\right)\right)} \log \left(1+|| \omega(t) \|_{L^{\infty}\left(B\left(0, R_{0}-\epsilon\right)\right)}\right)}
$$

This allows us to estimate the volume of the relevant super-level set of 
$|\omega|$ at time $t$,

$$
\begin{aligned}
& \operatorname{Vol}\left(\Lambda_{t}\left(\frac{1}{c_{1}}\|\omega(t)\|_{L^{\infty}\left(B\left(0, R_{0}-\epsilon\right)\right)}\right)\right) \\
& \leq \frac{c_{1} \log \left(c_{1}\right)\|\omega(t)\|_{L^{1}\left\{\{\omega(x, t) \mid \geq \lambda(t)\} \cap B\left(0, R_{0}-\epsilon\right)\right)}}{\|\omega(t)\|_{L^{\infty}\left(B\left(0, R_{0}-\epsilon\right)\right)} \log \left(1+\|\omega(t)\|_{L^{\infty}\left(B\left(0, R_{0}-\epsilon\right)\right)}\right.} \\
& \quad+\frac{c_{1}\|\omega(t) \log (1+|\omega(t)|)\|_{L^{1}\left(\{|\omega(x, t)| \geq \lambda(t)\} \cap B\left(0, R_{0}-\epsilon\right)\right)}}{\|\omega(t)\|_{L^{\infty}\left(B\left(0, R_{0}-\epsilon\right)\right)} \log \left(1+\|\omega(t)\|_{L^{\infty}\left(B\left(0, R_{0}-\epsilon\right)\right)}\right)} \\
& \leq \frac{K I_{0}(t)}{\|\omega(t)\|_{L^{\infty}\left(B\left(0, R_{0}-\epsilon\right)\right)} \log \left(1+\|\omega(t)\|_{L^{\infty}\left(B\left(0, R_{0}-\epsilon\right)\right)}\right)}
\end{aligned}
$$

where we have set,

$$
I_{0}(t)=\int_{\{|\omega(x, t)| \geq \lambda(t)\} \cap B\left(0, R_{0}-\epsilon\right)}|\omega(x, t)| \log (1+|\omega(x, t)|) d x,
$$

and have introduced a time-independent constant $K$ which depends on the fixed values $c_{1}$ and $R_{0}$ as well as the a priori finite quantity,

$$
\sup _{0 \leq t \leq T}\|\omega(\cdot, t)\|_{L^{1}\left(\{|\omega(x, t)| \geq \lambda(t)\} \cap B\left(0, R_{0}-\epsilon\right)\right)} .
$$

The second step ensures we can control $I_{0}(t)$ in terms of the finite bounds appearing in part (i) of the theorem. Tacitly summing over $j$, we have,

$$
I_{0}(t) \leq C \int_{B\left(0, R_{0}-\epsilon\right)}\left|\omega_{j}\right| \log \left(1+\sqrt{\omega_{1}^{2}+\omega_{2}^{2}+\omega_{3}^{2}}\right) d x .
$$

An explicit reduction illustrates the argument (for simplicity we take $j=1$ and integrals over the indicated sets intersected with $B\left(0, R_{0}-\epsilon\right)$ ),

$$
\begin{aligned}
& \int\left|\omega_{1}\right| \log \left(1+\sqrt{\omega_{1}^{2}+\omega_{2}^{2}+\omega_{3}^{2}}\right) d x \\
& \leq \int_{\omega_{1}^{2} \leq \omega_{2}^{2}+\omega_{3}^{2}} \sqrt{\omega_{2}^{2}+\omega_{3}^{2}} \log \left(1+\sqrt{2\left(\omega_{2}^{2}+\omega_{3}^{2}\right)}\right) d x \\
& \quad+\int_{\omega_{1}^{2}>\omega_{2}^{2}+\omega_{3}^{2}}\left|\omega_{1}\right| \log \left(1+\sqrt{2}\left|\omega_{1}\right|\right) d x .
\end{aligned}
$$


Applying the same reasoning to the first integral above and then repeating for all values of $j$ eventually yields,

$$
I_{0}(t) \leq C \int w_{i} d x+C \int w_{i} \log w_{i} d x \leq C M_{i}
$$

The energy inequality-type construction used to prove the previous theorem also works if we substitute $\log ^{m} \omega_{k}$ (as defined in Section 4.3.1) in place of $\log w_{k}$. This allows the application of amenable blow-up rates of order 1 in conjunction with Lemma 4.3.3.c. To ensure things are meaningful, we modify our definition of $q$ so that $q(y)=\sqrt{e+y^{2}}$ (so, now, $w_{k}=\sqrt{e+\omega_{k}^{2}}$ ) and refer to (4.3.1) to define $w_{k,+}$ and $w_{k,-}$.

Theorem 4.3.5. Let $u$ be a Leray solution to $(4.3 .2)$ on $\mathbb{R}^{3} \times(0, \infty)$, and suppose additionally that $\omega_{0}=\nabla \times u_{0} \in L^{1} \cap L^{2}$. Denote by $T$ the first singular time and by $S$ the singular set of $\omega$ at time $T$. Fix positive values $R_{0}$ and $\epsilon$ so that $\emptyset \neq S \cap B\left(0, R_{0}\right) \subset B\left(0, R_{0}-\epsilon\right)$ - i.e. there are singular points in $B\left(0, R_{0}\right)$ but all such points are in $B\left(0, R_{0}-\epsilon\right)$ - and let $Q_{T}(S)=Q_{T}\left(0, R_{0}, S\right)$.

(i) If $\left.w_{k,+}\right|_{Q_{T}(S)}$ and $\left.w_{k,-}\right|_{Q_{T}(S)}$ each exhibit amenable blow-up rates of order 1 in $Q_{T}(S)$, then there exists a positive value $M_{k}$ so that,

$$
\sup _{t \in[0, T]} \int_{B\left(0, R_{0}-\epsilon\right)}\left|\omega_{k}(x, t)\right| \log \log \left(\sqrt{e+\left|\omega_{k}(x, t)\right|^{2}}\right) d x<M_{k} .
$$

(ii) Let $c_{1}$ ba a fixed constant which is greater than 1. If, for all $k$, the premises of part (i) are satisfied, then there exists a positive value $M_{0}$ so that, for $t \in[0, T]$,

$$
\begin{aligned}
& \operatorname{Vol}\left(\Lambda_{t}\left(\frac{1}{c_{1}}\|\omega(t)\|_{L^{\infty}\left(B\left(0, R_{0}-\epsilon\right)\right)}\right)\right) \\
& \leq \frac{M_{0}}{\|\omega(t)\|_{L^{\infty}\left(B\left(0, R_{0}-\epsilon\right)\right)} \log \log \left(e+\|\omega(t)\|_{L^{\infty}\left(B\left(0, R_{0}-\epsilon\right)\right)}\right)} .
\end{aligned}
$$

Proof. Multiplying equation (4.3.3) by,

$$
\psi q^{\prime}\left(w_{k}\right)\left(\log \log w_{k}+w_{k}(\log \log )^{\prime} w_{k}\right),
$$


we obtain the evolution of $\psi w_{k} \log \log w_{k}$. The point-wise estimates in (4.3.5) adapt directly to the function $\log \log (y)$ (indeed, they adapt to any number of such self-compositions of the logarithm) and, after integrating in space and time, all of the estimates and cancellations from the previous proof - except those involving the vortex stretching term - can be duplicated directly. We thus obtain, denoting by $R$ some a priori finite quantity,

$$
\int\left(\psi w_{k} \log \log w_{k}\right)(t) d x \leq\left|\int_{0}^{t} \int \psi \omega \cdot \nabla u_{k} \frac{\omega_{k}}{w_{k}} \log \log w_{k} d x d s\right|+R
$$

and, using Lemma 4.3.3.c., we are able to pull out of the integral the uniformly-in-time bounded quantity,

$$
\sup _{t}\left\|\psi \frac{\omega_{k}}{w_{k}} \log \log w_{k}\right\|_{B M O},
$$

and conclude exactly as in the previous proof.

\subsection{A geometric scenario exhibiting loga- rithmically subcritical anisotropic dif- fusion}

Consider a weak (distributional) Leray solution $u$ on $\mathbb{R}^{3}$. The vorticity analogue of the Leray's a priori bound on the energy was presented in [31] where, assuming that the initial vorticity is in $L^{1}$ (or, more generally, a bounded measure), it is shown that the $L^{1}$-norm of the vorticity remains bounded on any finite time-interval. In this section our goal is to obtain a spatially localized $L \log L$ bound on the vorticity under a suitable assumption on the structural blow-up of the vorticity direction $\xi=\frac{\omega}{|\omega|}$.

Fix a ball $B\left(0, R_{0}\right) \subset \mathbb{R}^{3}$, and consider a test function $\psi$ supported in $B=B\left(0,2 R_{0}\right)$ with $\psi=1$ on $B\left(0, R_{0}\right)$ and $|\nabla \psi(x)| \leq c \frac{1}{R_{0}} \psi^{\delta}(x)$ for some $\delta>0$. Let $w=\sqrt{1+|\omega|^{2}}$. We aim to control the evolution of $\psi w \log w$; by Stein's lemma [100], this is essentially equivalent to controlling the $L^{1}$-norm of $M w$. 
For simplicity of the exposition, we assume that the initial vorticity is also in $L^{2}$ and that $T>0$ is the first (possible) blow-up time. This way, the solution in view is smooth on $(0, T)$ and we can focus on obtaining a supremum-in-time type bound.

Theorem 4.4.1. Let $u$ be a Leray solution to the 3D NSE. Assume that the initial vorticity $\omega_{0}$ is in $L^{1} \cap L^{2}$, and that $T>0$ is the first (possible) blow-up time. Suppose that

$$
\sup _{t \in(0, T)}\|(\psi \xi)(\cdot, t)\|_{\widehat{b m o} \frac{1}{|\log r|}}<\infty
$$

Then,

$$
\sup _{t \in(0, T)} \int \psi(x) w(x, t) \log w(x, t) d x<\infty .
$$

Remark 4.4.1. Since $\omega_{0}$ is in $L^{1}$, in addition to the Leray's a priori bounds on $u$,

$$
\sup _{0<t<T}\|u(\cdot, t)\|_{L^{2}}<\infty \text { and } \int_{0}^{T} \int_{\mathbb{R}^{3}}|\nabla u(x, t)|^{2} d x d t<\infty,
$$

the following a priori bounds on $\omega$ are also at our disposal [31],

$$
\sup _{t}\|\omega(\cdot, t)\|_{L^{1}}<\infty \text { and } \int_{0}^{T} \int_{\mathbb{R}^{3}}|\nabla \omega(x, t)|^{\frac{4}{3+\epsilon}} d x d t<\infty .
$$

Proof. Setting $q(y)=\sqrt{1+|y|^{2}}$, the evolution of $w=\sqrt{1+|\omega|^{2}}$ satisfies the following partial differential inequality $([31])$,

$$
\partial_{t} w-\Delta w+(u \cdot \nabla) w \leq \omega \cdot \nabla u \cdot \frac{\omega}{w}
$$

Since our goal is to control the evolution of $\psi w \log w$ we multiply (4.4.1) by $\psi(1+\log w)$. Here is the calculus corresponding to each of the four terms:

1. Time derivative,

$$
\partial_{t} w \times \psi(1+\log w)=\partial_{t}(\psi w \log w)
$$


2. Laplacian,

$$
\begin{aligned}
- & \Delta w \times \psi(1+\log w) \\
= & -\Delta(\psi w \log w)+\Delta \psi w \log w \\
& +\psi \frac{1}{w} \sum_{i}\left(\partial_{i} w\right)^{2}+2 \sum_{i} \partial_{i} \psi \partial_{i} w(1+\log w),
\end{aligned}
$$

3. Advection,

$$
\begin{aligned}
& (u \cdot \nabla) w \times \psi(1+\log w) \\
& =\sum_{i} u_{i} \partial_{i} w \psi(1+\log w) \\
& =\sum_{i}\left(\partial_{i}\left(u_{i} w \psi(1+\log w)\right)-u_{i} w \partial_{i} \psi(1+\log w)-u_{i} \psi \partial_{i} w\right) \\
& =\sum_{i}\left(\partial_{i}\left(u_{i} w \psi(1+\log w)\right)\right. \\
& \left.\quad-u_{i} w \partial_{i} \psi(1+\log w)-\partial_{i}\left(u_{i} \psi w\right)+\left(u_{i} \partial_{i} \psi w\right)\right)
\end{aligned}
$$

4. Vortex stretching,

$$
\begin{aligned}
& \omega \cdot \nabla u \cdot \frac{\omega}{w} \times \psi(1+\log w) \\
& =\omega \cdot \nabla u \cdot \psi \frac{\omega}{|\omega|}(1+\log w)+\omega \cdot \nabla u \cdot \psi\left(\frac{\omega}{w}-\frac{\omega}{|\omega|}\right)(1+\log w) .
\end{aligned}
$$

Integrating in time and space, dropping the zero and the positive terms, and estimating the remaining terms in the straightforward fashion via the Hölder and Sobolev inequalities, these formulas lead us to,

$$
\begin{aligned}
& I(\tau) \equiv \int \psi(x) w(x, \tau) \log w(x, \tau) d x \\
& \leq I(0)+c \int_{0}^{\tau} \int_{\mathbb{R}^{3}} \omega \cdot \nabla u \cdot \psi \xi \log w d x d t+R,
\end{aligned}
$$

where $\tau$ in $[0, T)$ and $R$ is a priori bounded.

To take the advantage of Coifman-Rochberg's estimate, we decompose the logarithmic factor as,

$$
\log w=\log \frac{w}{\mathcal{M} w}+\log \mathcal{M} w .
$$


Denoting $\int_{0}^{\tau} \int_{\mathbb{R}^{3}} \omega \cdot \nabla u \cdot \psi \xi \log w d x d t$ by $J$, this yields $J=J_{1}+J_{2}$ where,

$$
J_{1}=\int_{0}^{\tau} \int_{\mathbb{R}^{3}} \omega \cdot \nabla u \cdot \psi \xi \log \frac{w}{\mathcal{M} w} d x d t,
$$

and,

$$
J_{2}=\int_{0}^{\tau} \int_{\mathbb{R}^{3}} \omega \cdot \nabla u \cdot \psi \xi \log \mathcal{M} w d x d t .
$$

For $J_{1}$, we use the pointwise inequality,

$$
w \log \frac{w}{\mathcal{M} w} \leq \mathcal{M} w-w
$$

(a consequence of the pointwise inequality $\mathcal{M} f \geq f$, and the inequality $e^{x-1} \geq x$ for $x \geq 1$ ). This leads to,

$$
J_{1} \leq \int_{0}^{\tau} \int_{\mathbb{R}^{3}}|\nabla u|(\mathcal{M} w-w) \psi d x d t,
$$

which is a priori bounded by the Cauchy-Schwarz inequality and the $L^{2}$-maximal theorem.

For $J_{2}$, we have the following string of inequalities,

$$
\begin{aligned}
J_{2} \leq & c \int_{0}^{\tau}\|\omega \cdot \nabla u\|_{\mathfrak{h}^{1}}\|\psi \xi \log \mathcal{M} w\|_{b m o} d t \\
\leq & c \int_{0}^{\tau}\|\omega \cdot \nabla u\|_{\mathcal{H}^{1}}\|\psi \xi \log \mathcal{M} w\|_{\widehat{b m o}} d t \\
\leq & c \int_{0}^{\tau}\|\omega\|_{2}\|\nabla u\|_{2}\left(\|\psi \xi\|_{\infty}+\|\psi \xi\|_{\widetilde{b m o}}\right) \\
& \cdot\left(\|\log \mathcal{M} w\|_{B M O}+\|\log \mathcal{M} w\|_{1}\right) d t \\
\leq & c \sup _{t \in(0, T)}\left\{\left(1+\|\psi \xi\|_{\widetilde{b m o}}\right)\left(\|\log \mathcal{M} w\|_{B M O}+\|\log \mathcal{M} w\|_{1}\right)\right\} \\
& \quad \cdot \quad \int_{0}^{T} \int_{\mathbb{R}^{3}}|\nabla u|^{2} d x d t \\
\leq & c\left(1+\sup _{t \in(0, T)}\|\omega\|_{1}\right) \sup _{t \in(0, T)}\left(1+\|\psi \xi\|_{\overparen{b m o}}\right) \int_{0}^{T} \int_{\mathbb{R}^{3}}|\nabla u|^{2} d x d t
\end{aligned}
$$

by $\mathfrak{h}^{1}$ - bmo duality, the Div-Curl Lemma, the pointwise $\widetilde{b m o}$-multiplier theorem, the Coifman-Rochberg's estimate, and the bound (4.2.2). This completes the proof of the $L \log L$ estimate. 


\section{Bibliography}

[1] L.V. Ahlfors. Conformal invariants: topics in geometric function theory. AMS Chelsea Pub., 2010.

[2] H. Aluie. Hydrodynamic and Magnetohydrodynamic Turbulence: Invariants, cascades, and locality. Phd thesis, Johns Hopkins University, 2009.

[3] H. Aluie and G. L. Eyink. Scale locality of magnetohydrodynamic turbulence. Phys. Rev. Lett., 104(8):081101, 2010.

[4] H. Bae, A. Biswas, and E. Tadmor. Analyticity and decay estimates of the Navier-Stokes equations in critical Besov spaces. Arch. Ration. Mech. Anal., 205(3):963-991, 2012.

[5] H. Beirão da Veiga and L. C. Berselli. On the regularizing effect of the vorticity direction in incompressible viscous flows. Differential Integral Equations, 15(3):345, 2002.

[6] A. Beresnyak. Basic properties of magnetohydrodynamic turbulence in the inertial range. Mon. Not. R. Astron. Soc., 422(4):3495, 2012.

[7] A. Beresnyak and Lazarian A. Strong imbalanced turbulence. ApJ, 682(2):1070, 2010.

[8] D. Biskamp. Magnetohydrodynamic Turbulence. Cambridge University Press, 2003.

[9] D. Biskamp and E. Schwartz. On two-dimensional magnetohydrodynamic turbulence. Phys. Plasmas, 8(7):3282, 2001. 
[10] A. Biswas and D. Swanson. Gevrey regularity of solutions to the 3 -D Navier-Stokes equations with weighted $l_{p}$ initial data. Indiana Univ. Math. J., 56(3):1157-1188, 2007.

[11] W. Blumen. Uniform potential vorticity flow. part i. theory of wave interaction and two-dimensional turbulence. J. Atmos. Sci, 25:774-783, 1978.

[12] S. Boldyrev. On the spectrum of magnetohydrodynamic turbulence. Astrophys. J. Lett., 626(1):L37, 2005.

[13] J. E. Borovsky. Flux tube texture of the solar wind: Strands of the magnetic carpet at 1 au? J. of Geophys. Res., 113(A):8, 2008.

[14] Z. Bradshaw. Geometric measure-type regularity criteria for the 3D magnetohydrodynamical system. Nonlinear Anal., 75(16):6180-6190, 2012.

[15] Z. Bradshaw and Z. Grujić. Energy cascades in physical scales of 3d incompressible magnetohydrodynamical turbulence. J. Math. Phys., 54:093503, 2013.

[16] Z. Bradshaw and Z. Grujić. On the transport and concentration of enstrophy in 3d magnetohydrodynamic turbulence. Nonlinearity, 26:2373, 2013.

[17] Z. Bradshaw and Z. Grujić. Blow-up scenarios for 3d nse exhibiting sub-criticality with respect to the scaling of one-dimensional local sparsenes. J. Math. Fluid Mech., (to appear).

[18] Z. Bradshaw and Z. Grujić. A spatially localized $1 \log l$ estimate on the vorticity in the $3 \mathrm{~d}$ nse. Indiana Univ. Math. J., (to appear).

[19] R. Bruno, V. Carbone, P. Veltri, E. Pietropaolo, and B. Bavassano. Identifying intermittency events in the solar wind. Planetary and Space Science, 49(12):1201, 2001.

[20] L. Caffarelli, R. Kohn, and L. Nirenberg. Partial regularity of suitable weak solutions of the Navier-Stokes equations. Comm. Pure Appl. Math., 35(6):771, 1982. 
[21] L. Caffarelli and L. Silvestre. An extension problem related to the fractional laplacian. Comm. Partial Differential Equations, 32(7-9):1245-1260, 2007.

[22] L. Caffarelli and A. Vasseur. Drift diffusion equations with fractional diffusion and the quasi-geostrophic equation. Ann. of Math. (2), 171(3):1903-1930, 2010.

[23] D. C. Chang, G. Dafni, and E.M. Stein. Hardy spaces, BMO, and boundary value problems for the Laplacian on a smooth domain in $\mathbb{R}^{n}$. Trans. Amer. Math. Soc., 351(4):1605-1661, 1999.

[24] C. Chen, R. Strain, H.-T. Yau, and T.-P. Tsai. Lower bound on the blow-up rate of the axisymmetric Navier-Stokes equations. Int. Math. Res. Not. IMRN, (9):16-31, 2008.

[25] C. Chen, R. M. Strain, T. Tsai, and H.T. Yau. Lower bounds on the blow-up rate of the axisymmetric Navier-Stokes equations. II. Comm. Partial Differential Equations, 34(1-3):203-232, 2009.

[26] A. Cheskidov, P. Constantin, S. Friedlander, and R. Shvydkoy. Energy conservation and Onsager's conjecture for the Euler equations. Nonlinearity, 21(6):1233, 2008.

[27] A. Cheskidov and R. Shvydkoy. Euler equations and turbulence: Analytical approach to intermittency. SIAM Journal on Mathematical Analysis, 46(1):353-374, 2014.

[28] A. J. Chorin. Vorticity and Turbulence. Number v. 103 in Applied Mathematical Sciences. Springer, 1994.

[29] R. Coifman, P.L. Lions, Y. Meyer, and S. Semmes. Compensated compactness and Hardy spaces. J. Math. Pures Appl. (9), 72(3):247-286, 1993.

[30] R. R. Coifman and R. Rochberg. Another characterization of bmo. Proc. Amer. Math. Soc., 79(2):249254, 1980.

[31] P. Constantin. Navier-stokes equations and area of interfaces. Comm. Math. Phys., page 241, 1990. 
[32] P. Constantin. Geometric statistics in turbulence. SIAM Rev., 36(1):73, 1994.

[33] P. Constantin. Energy spectrum of quasi-geostrophic turbulence. Phys. Rev. Lett., 89:184501, 2002.

[34] P. Constantin and C. Fefferman. Direction of vorticity and the problem of global regularity for the Navier-Stokes equations. Indiana Univ. Math. J., 42(3):775, 1993.

[35] P. Constantin and C. Foias. Navier-Stokes equations. Chicago Lectures in Mathematics. University of Chicago Press, Chicago, IL, 1988.

[36] P. Constantin, M. Lai, R. Sharma, Y. Tseng, and J. Wu. New numerical results for the surface quasi-geostrophic equation. $J$. Sci. Comput., 50(1):1-28, 2012.

[37] P. Constantin, A. J. Majda, and E. G. Tabak. Singular front formation in a model for quasigeostrophic flow. Phys. Fluids, 6(1):9-11, 1994.

[38] P. Constantin, I. Procaccia, and D. Segel. On the creation and dynamics of vortex structures in three-dimensional turbulence. Phys. Rev. E, 51(4):3207, 1995.

[39] P. Constantin and J. Wu. Regularity of Hölder continuous solutions of the supercritical quasi-geostrophic equation. Ann. Inst. H. Poincaré Anal. Non Linéaire, 25(6):1103-1110, 2008.

[40] R. Dascaliuc and Z. Grujić. Energy cascades and flux locality in physical scales of the 3D Navier-Stokes equations. Comm. Math. Phys., 305(1):199, 2011.

[41] R. Dascaliuc and Z. Grujić. Anomalous dissipation and energy cascade in 3D inviscid flows. Comm. Math. Phys., 309(3):757, 2012 .

[42] R. Dascaliuc and Z. Grujić. Dissipation anomaly and energy cascade in 3D incompressible flows. C. R. Math. Acad. Sci. Paris, 350(3-4):199, 2012. 
[43] R. Dascaliuc and Z. Grujić. Vortex stretching and criticality for the three-dimensional Navier-Stokes equations. J. Math. Phys., 53(11):115613, 2012.

[44] R. Dascaliuc and Z. Grujić. Vortex stretching and criticality for the three-dimensional Navier-Stokes equations. J. Math. Phys., 53,2012 .

[45] R. Dascaliuc and Z. Grujić. Coherent vortex structures and 3D enstrophy cascade. Comm. Math. Phys., 317(2):547, 2013.

[46] O. Debliquy, M. Verma, and D. Carati. Energy fluxes and shellto-shell transfers in three-dimensional decaying magnetohydrodynamical turbulence. Phys. Plasmas, 12(4):042309, 2005.

[47] G. L. Eyink. Locality of turbulent cascades. Phys. D, 207(12):91, 2005.

[48] G. L. Eyink and H. Aluie. The breakdown of Alfvén's theorem in ideal plasma flows: Necessary conditions and physical conjectures. Phys. D, 223(1):82, 2006.

[49] M. Sodin F. Nazarov and A. Vol'berg. The geometric KannanLovaśz-Simonovits lemma, dimension-free estimates for the distribution function of the values of polynomials, and the distribution of the zeros of random analytic functions. St. Petersburg Math. J., 14:351-366, 2003.

[50] C. Fefferman. Characterizations of bounded mean oscillation. Bull. Amer. Math. Soc., 77:587-588, 1971.

[51] C. Fefferman and E. M. Stein. $H^{p}$ spaces of several variables. Acta Math., 129(3-4):137-193, 1972.

[52] C. Foias, O. Manley, R. Rosa, and R. Temam. Estimates for the energy cascade in three-dimensional turbulent flows. C. R. Acad. Sci. Paris Sér. I Math., 333(5):499, 2001.

[53] C. Foias, O. Manley, R. Rosa, and R. Temam. Navier-Stokes equations and turbulence, volume 83 of Encyclopedia of Mathematics and its Applications. Cambridge University Press, Cambridge, 2001. 
[54] C. Foias and R. Temam. Gevrey class regularity for the solutions of the Navier-Stokes equations. J. Funct. Anal., 87(2):359-369, 1989 .

[55] U. Frisch. Turbulence. Cambridge University Press, Cambridge, 1995. The legacy of A. N. Kolmogorov.

[56] S. Galtier, A. Pouquet, and A. Mangeney. On spectral scaling laws for incompressible anisotropic magnetohydrodynamic turbulence. Phys. Plasmas, 12(9):092310, 2005.

[57] P. Goldreich and S. Sridhar. Toward a theory of interstellar turbulence. i. weak Alfvénic turbulence. Astrophys. J., 432:612, 1994.

[58] P. Goldreich and S. Sridhar. Toward a theory of interstellar turbulence. ii. strong Alfvénic turbulence. Astrophys. J., 438:763, 1995.

[59] A. Greco, P. Chuychai, W. H. Matthaeus, W. Servidio, and P. Dmitruk. Intermittent mhd structures and classical discontinuities. Geophys. Res. Lett., 35(L):19111, 2008.

[60] A. Greco, W. H. Matthaeus, W. Servidio, P. Chuychai, and P. Dmitruk. Statistical analysis of discontinuities in solar wind ace data and comparison with intermittent mhd turbulence. Astrophys. J., 691(L):111, 2009.

[61] Z. Grujić. A geometric measure-type regularity criterion for solutions to the 3D Navier-Stokes equations. Nonlinearity, 26:289296, 2013.

[62] Z. Grujić and I. Kukavica. Space analyticity for the Navier-Stokes and related equations with initial data in $L^{p}$. J. Funct. Anal., 152(2):447-466, 1998.

[63] Z. Grujić and I. Kukavica. Space analyticity for the nonlinear heat equation in a bounded domain. J. Differential Equations, 154(1):42-54, 1999.

[64] R. Guberović. Smoothness of koch-tataru solutions to the navier-stokes equations revisited. Discrete Contin. Dyn. Syst., 27(1):231-236, 2010. 
[65] C. He and Z. Xin. On the regularity of weak solutions to the magnetohydrodynamic equations. J. Differential Equations, $213(2): 235,2005$.

[66] C. He and Z. Xin. Partial regularity of suitable weak solutions to the incompressible magnetohydrodynamic equations. J. Func. Anal., 227(1):113, 2005.

[67] I. Held, R. Pierrehumbert, S. Garner, and K. Swanson. Surface quasi-geostrophic dynamics. J. Fluid Mech., 282:1-20, 1995.

[68] T. Hou and R. Li. Nonexistence of locally self-similar blow-up for the 3D incompressible Navier-Stokes equations. Discrete Contin. Dyn. Syst., 18(4):637-642, 2007.

[69] P. Iroshnikov. Turbulence of a conducting fluid in a strong magnetic field. Sov. Astron., 7:566, 1964.

[70] T. Iwaniec and A. Verde. On the operator $L(f)=f \log |f| . \quad J$. Funct. Anal., 169(2):391-420, 1999.

[71] S. Janson. On functions with conditions on the mean oscillation. 14(1-2):189-196, 1976.

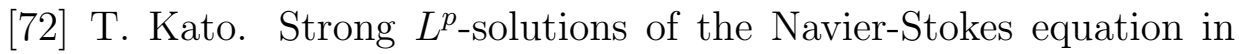
$\mathbf{R}^{m}$, with applications to weak solutions. Math. Z., 187(4):471480, 1984.

[73] R. Kinney, J. C. McWilliams, and T. Tajima. Coherent structures and turbulent cascades in two-dimensional incompressible magnetohydrodynamic turbulence. Phys. Plasmas, 2(10):3623, 1995 .

[74] G. Koch, N. Nadirashvili, G. A. Seregin, and V. Šverák. Liouville theorems for the Navier-Stokes equations and applications. Acta Math., 203(1):83-105, 2009.

[75] R. H. Kraichnan. Inertial range spectrum in hydromagnetic turbulence. Phys. Fluids, 8(7):1385, 1966.

[76] I. Kukavica. On the dissipative scale for the Navier-Stokes equation. Indiana Univ. Math. J., 48(3):1057-1081, 1999. 
[77] I. Kukavica. On local uniqueness of weak solutions of the navierstokes system with bounded initial data. J. Differential Equations, 194:39-50, 2003.

[78] I. Kukavica and V. Vicol. The domain of analyticity of solutions to the three-dimensional Euler equations in a half space. Discrete Contin. Dyn. Syst., 29(1):285-303, 2011.

[79] I. Kukavica and V. Vicol. On the analyticity and Gevrey-class regularity up to the boundary for the Euler equations. Nonlinearity, 24(3):765-796, 2011.

[80] I. Kukavica and V. Vicol. On the analyticity and Gevrey-class regularity up to the boundary for the Euler equations. Nonlinearity, 24(3):765-796, 2011.

[81] P.-L. Lions. Mathematical topics in fluid mechanics. Vol. 1, volume 3 of Oxford Lecture Series in Mathematics and its Applications. The Clarendon Press Oxford University Press, New York, 1996. Incompressible models, Oxford Science Publications.

[82] W. H. Matthaeus, D. C. Montgomery, M. Wan, and S. Servidio. A review of relaxation and structure in some turbulent plasmas: magnetohydrodynamics and related models. Journal of Turbulence, 13(37):1, 2012.

[83] V. G. Mazya and T. O. Shaposhnikova. Theory of multipliers in spaces of differentiable functions. Monographs and studies in mathematics, 23, 1985.

[84] A. Mazzucato and V. Nistor. Mapping properties of heat kernels, maximal regularity, and semi-linear parabolic equations on noncompact manifolds. J. Hyperbolic Differ. Equ., 3(4):599-629, 2006 .

[85] C. Miao, B. Yuan, and B. Zhang. Well-posedness for the incompressible magneto-hydrodynamic system. Math. Methods Appl. Sci., 30:961-976, 2007.

[86] W.C. Müller and R. Grappin. Spectral energy dynamics in magnetohydrodynamic turbulence. Phys. Rev. Lett., 95:114502, Sep 2005 . 
[87] E. Nakai and K. Yabuta. Pointwise multipliers for functions of bounded mean oscillations. J. Math. Soc. Japan, 37(2):207-218, 1985 .

[88] J. Nečas, M. Růžička, and V. Šverák. On Leray's self-similar solutions of the Navier-Stokes equations. Acta Math., 176(2):283294, 1996.

[89] R. Nevanlinna. Analytic Functions. Springer-Verlag, 1970.

[90] J. Perez and S. Bolydrev. Strong magnetohydrodynamic turbulence with cross helicity. Phys. Plasmas, 17(055903):1, 2010.

[91] J. C. Perez, J. Mason, S. Boldyrev, and F. Cattaneo. On the energy spectrum of strong magnetohydrodynamic turbulence. Phys. Rev. X, 2:041005, Oct 2012.

[92] R Pierrehumbert, I. Held, and K. Swanson. Spectra of local and nonlocal two-dimensional turbulence. Chaos, solitions and fractals, 4(6):1111, 1994.

[93] J. J. Podesta and A. Bhattacharjee. Theory of incompressible magnetohydrodynamic turbulence with scale-dependent alignment and cross-helicity. Astrophys. J., 718(2):1151, 2010.

[94] D. Pontin, A. Bhattacharjee, and K. Galsgaard. Current sheet formation and nonideal behavior at three-dimensional magnetic null points. Phys. Plasmas, 14(052106):1, 2007.

[95] G. Seregin and V. Šverák. On type I singularities of the local axi-symmetric solutions of the Navier-Stokes equations. Comm. Partial Differential Equations, 34(1-3):171-201, 2009.

[96] M. Sermange and R. Temam. Some mathematical questions related to the MHD equations. Comm. Pure Appl. Math., 36(5):635, 1983.

[97] S. Servidio, P. Dmitruk, A. Greco, M. Wan, S. Donato, P. A. Cassak, M. A. Shay, V. Carbone, and W. H. Matthaeus. Magnetic reconnection as an element of turbulence. Nonlinear Processes in Geophysics, 18(5):675-695, 2011. 
[98] R. Shvydkoy. A study of energy concentration and drain in incompressible fluids. Nonlinearity, 26(2):425-436, 2013.

[99] A. Y. Solynin. Ordering of sets, hyperbolic metrics, and harmonic measures. J. Math. Sci., 95:2256-2266, 1999.

[100] E. M. Stein. Note on the class L log L. Studia Math., 32:305-310, 1969.

[101] E. M. Stein. Singular Integrals. Princeton Univ. Press, Princeton, 1970.

[102] E. M. Stein, editor. Beijing lectures in harmonic analysis, volume 112 of Annals of Mathematics Studies, Princeton, NJ, 1986. Princeton University Press.

[103] E. M. Stein. Harmonic analysis: real-variable methods, orthogonality, and oscillatory integrals, volume 43 of Princeton Mathematical Series. Princeton University Press, Princeton, NJ, 1993.

[104] Roger Temam. Navier-Stokes equations. AMS Chelsea Publishing, Providence, RI, 2001. Theory and numerical analysis, Reprint of the 1984 edition.

[105] T.-P. Tsai. Erratum: "On Leray's self-similar solutions of the Navier-Stokes equations satisfying local energy estimates" [Arch. Rational Mech. Anal. 143 (1988), no. 1, 29-51; MR1643650 (99j:35171)]. Arch. Ration. Mech. Anal., 147(4):363, 1999.

[106] A. Vorobev, O. Zikanov, P. Davidson, and B. Knaepen. Anisotropy of magnetohydrodynamic turbulence at low magnetic Reynolds number. Phys. Fluids, 17(12):125105, 2005.

[107] J. Wu. Analytic results related to magneto-hydrodynamic turbulence. Phys. D, 136(3-4):353, 2000.

[108] J. Wu. Bounds and new approaches for the 3D MHD equations. J. Nonlinear Sci., 12(4):395, 2002.

[109] B. Yuan. On the blow-up criterion of smooth solutions to the mhd system in bmo space. Acta Math. Appl. Sin. Engl. Set., 22(3):413-418, 2006. 
[110] V. Zhdankin, S. Boldyrev, and J. Mason. Distribution of magnetic discontinuities in the solar wind and in magnetohydrodynamic turbulence. The Astrophysical Journal Letters, 760(2):L22, 2012. 